\title{
BROADBAND SPECTRAL INVESTIGATIONS OF MAGNETAR BURSTS
}

\author{
by \\ DEMET KIRMIZIBAYRAK
}

Submitted to the Graduate School of Engineering and Natural Sciences in partial fulfillment of the requirements for the degree of

Master of Science

Sabanci University

May 2017 


\section{BROADBAND SPECTRAL INVESTIGATIONS OF MAGNETAR BURSTS}

APPROVED BY:

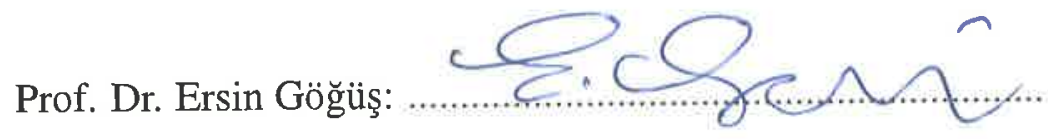

(Thesis Supervisor)

Prof. Dr. M. Ali Alpar:

Assoc. Prof. Dr. Tolga Guiver:

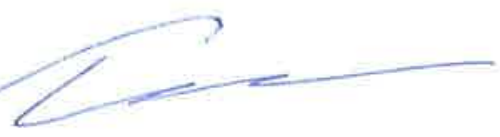

Assoc. Prof. Dr. Tolga Giver: ...

DATE OF APPROVAL: ........... 5,2017 
(c) DEMET KIRMIZIBAYRAK 2017

All rights reserved. 


\section{ACKNOWLEDGEMENTS}

Firstly, I would like to express my gratitude to my advisor Prof. Ersin Gogus for his guidance and

support. Interacting with him in research has broadened my vision and helped me guide and appease my curiosity. His guidance let me follow my academic goals, for this I am most thankful. I would also like to express my appreciation to Dr. Sinem Sasmaz Mus and Assoc. Prof. Yuki Kaneko. Their support and guidance has been very valuable on my research activities.

I would also like to thank Prof. M. Ali Alpar, Prof. Kalvir Dhuga, Assoc. Prof. Tolga Guver, Assoc. Prof. Unal Ertan and Prof. Inanc Adagideli for supporting me with their valuable comments and instruction.

I must express my gratitude to Prof. Alkan Kabakcioglu for his valuable comments and his patient guidance and support throughout my career.

I acknowledge support and funding from the Scientific and Technological Research Council of Turkey (TUBITAK, project no: 113R031).

I thank my brother Can Kirmizibayrak for setting an inspiration and my friends for making this journey more enjoyable. Finally, and most importantly, I would like to thank my parents Hatice and Tayfun Kirmizibayrak for their persistent love, care and support. All my accomplishments do and will belong to them for they are the ones who so perfectly guided me in every step of the way with love. 


\title{
ABSTRACT
}

\section{BROADBAND SPECTRAL INVESTIGATIONS OF MAGNETAR BURSTS}

\author{
Demet Kirmizibayrak
}

Physics, M.Sc. Thesis, 2017

Supervisor: Prof. Ersin Göğüş

\section{Keywords: Magnetars, X-rays, Bursts, Spectral Analysis}

Magnetars are neutron stars whose variety of energetic emission mechanisms are thought to be governed by the decay of their extremely strong magnetic fields ( $\mathrm{B} \sim 10^{14} G$ ). Studies on radiative magnetar behaviour promise insight into emission mechanisms in highly magnetized regions as well as the formation, evolution and structure of neutron stars. In this thesis, we present our broadband (2-250 keV) spectral analysis of 42, 125 and 221 bursts from magnetar sources SGR J1550-5418, SGR 1900+14 and SGR 1806-20, respectively, detected with the Rossi X-ray Timing Explorer (RXTE) mission. We find that two blackbody functions $(\mathrm{BB}+\mathrm{BB})$, sum of two modified blackbody functions $(\mathrm{LB}+\mathrm{LB})$, sum of blackbody and powerlaw functions $(\mathrm{BB}+\mathrm{PO})$ and a power law with a high energy exponential cut-off (COMPT) all provide acceptable fits at similar levels. We report that when a $\chi^{2}$ comparison test is employed, 258 out of 388 bursts examined provided better fit statistics (lower $\chi^{2}$ within 0.05 significance) when fitted with the COMPT while 28 were better fitted with a sum of two blackbody functions. We performed numerical simulations to further constrain the best fitting model for each burst spectrum, and found that 69 out of 102 burst spectra with well-constrained parameters are significantly better described by the Comptonized model. We also found that 66 out of 102 these burst spectra are better described with LB+LB, which is employed in X-ray spectral modeling for the first time here, than $\mathrm{BB}+\mathrm{BB}$ and $\mathrm{BB}+\mathrm{PO}$. We also show a significant correlation between burst emission area and blackbody temperatures when $\mathrm{BB}+\mathrm{BB}$ fits are employed. We expand on the physical interpretation of these models and discuss our results in the framework of strongly magnetized neutron star case. 


\section{ÖZ}

\section{MAGNETAR PATLAMALARININ GENISŞ ENERJİ ARALIĞINDA TAYFSAL INNCELEMELERİ \\ Demet Kırmızıbayrak}

Fizik, Yüksek Lisans Tezi, 2017

Danışman: Prof. Ersin Göğüş

\section{Anahtar Kelimeler: Magnetarlar, X Işınları, Patlamalar, Tayfsal Analiz}

Magnetarlar, yaydıkları yüksek enerjilerdeki 1şımaların sahip oldukları yüksek manyetik alanların (B $\sim 10^{14} G$ ) bozunumu ile sağlandığı düşünülen nötron yıldızlarıdır. Magnetarların 1şıma karakteri üzerine yapılan çalışmalar, hem yüksek manyetik alanlarda ışık yayılımı hem de nötron yıldızlarının oluşumu, gelişimi ve yapısı üzerine bilgi edinme potansiyeli taşırlar. Bu tezde, SGR J1550-5418, SGR 1900+14 ve SGR 1806-20 kaynaklarının sırasıyla 42, 125 ve 221 adet tamamı Rossi X-ışını Zamanlama Kaşifi üzerindeki uydu teleskopları ile gözlenmiş patlamalarının sistematik olarak detaylı geniş dalga boyu aralığında tayfsal incelemesini gerçekleştirdik. İki kara ışıma modeli toplamı $(\mathrm{BB}+\mathrm{BB})$, iki Lyubarsky modeli toplamı $(\mathrm{LB}+\mathrm{LB})$, yüksek enerji kesitli güç modeli (COMPT) ve kara 1şıma ve güç modeli toplamlarının $(\mathrm{BB}+\mathrm{PO})$ bu patlamaları benzer düzeylerde kabul edilebilir uygunlukta betimlediğini gördük. $\chi^{2}$ karşılaştırma testi uyguladığımızda toplam 388 patlamanın 258 adetinin COMPT ile (0.05 anlamlılık düzeyinde daha düşük $\chi^{2}$ verecek şekilde), 28 adetinin ise iki kara 1şıma modeli toplamı ile istatistiksel olarak daha iyi betimlendiğini bulduk. Patlamaları en iyi açıklayan modeli bulmak için her bir patlama üzerinde nümerik simülasyonlar gerçekleştirdiğimizde, 102 hatası sınırlandırılmış patlamanın 69 tanesinin Compton modeli ile daha iyi betimlendiğini gördük. Kara 1şıma modelinin bir modifikasyonu olan ve X-1şını tayfsal analizinde ilk kez burada kullanılan iki Lyubarsky modelinin toplamının ise ayni 102 patlamanın 66 tanesini iki kara 1şıma modeli toplamından ve kara 1şıma ve güç modeli toplamından daha iyi betimlediğini gördük. Bu tezde ayrıca patlama yayılım alanı ve kara ışınım sıcaklığı arasında yüksek bir korelasyon tespit ettik. Son olarak, sonuçlarımızı kullandığımız modellerin fiziksel betimlemesi üzerine ve yüksek manyetik alanlı nötron yıldızları çerçevesinde tartıştık. 


\section{Contents}

1 Introduction 1

1.1 Neutron Stars . . . . . . . . . . . . . . . . . . . . . 1

1.1.1 General Properties . . . . . . . . . . . . . . . . 1

1.2 Magnetars . . . . . . . . . . . . . . . . . . . . 5

1.2.1 Theoretical Models . . . . . . . . . . . . . . . . . 6

1.2 .2 Bursts . . . . . . . . . . . . . . . . 9

1.2.3 Emission Mechanisms . . . . . . . . . . . . . . 10

1.3 Thesis Outline . . . . . . . . . . . . . . . . . . . . . 12

2 Observations and Burst Sample $\quad 13$

2.1 Instruments . . . . . . . . . . . . . . . . . . . . . . 13

2.2 Generation of Spectra . . . . . . . . . . . . . . . . . 14

3 Spectral Analysis and Results 33

3.1 Continuum Models . . . . . . . . . . . . . . . . . . . 33

3.2 Results . . . . . . . . . . . . . . . . . . . . 36

3.2.1 Descriptive Statistics of Spectral Parameters and Flux . . . . . . . . 37

3.2.2 Detailed Spectral Analysis Results . . . . . . . . . . . . . . . . 47

3.3 Peak Energy Comparison with Previous Studies . . . . . . . . . . . . 64

4 Discussion $\quad \mathbf{6 6}$

4.1 Identification of the Best Describing Model . . . . . . . . . . . . . 66

4.1 .1 Simulation Procedure . . . . . . . . . . . . . . . . . . 66

4.1 .2 Results . . . . . . . . . . . . . . . . . . . 67

4.1 .3 Implications . . . . . . . . . . . . . . . 71

4.2 Relation Between Temperature and Emission Area . . . . . . . . . . . 71

4.2 .1 Correlations . . . . . . . . . . . . . . . . . . 73

4.2.2 Power Indexes and Relationship with Flux . . . . . . . . . . . . 73

5 Summary and Conclusions $\quad 77$ 


\section{List of Figures}

1.1 Left Examples of neutron star equations of state (pressure as a function of density) proposed under different assumptions (for hadronic matter and strange quark matter (SQM)) Right Mass and radius relationships inferred from different equations of states proposed. Figure is taken from Özel \& Freire 2016 . . . . . . . . . . . . . . 3

1.2 Cooling curve (log Temperature $(\mathrm{K})$ vs log time (yrs)) of a sample of neutron stars. Figure is taken from Yakovlev et. al. (2011) . . . . . . . . . . . . . . . 4

$1.3 \dot{P}$ vs. P plot of neutron stars. Squares represent pulsars colored by magnetic field strength. Red stars indicate magnetars separated by the grey solid line indicating magnetic field above $4.4 \times 10^{13}$ G. Figure is taken from Rea et. al. (2010) . . . . . 5

1.4 Depiction of the trapped fireball in a corona of electron-positron pairs. Figure is taken from Thompson \& Duncan $2001 \ldots \ldots$. . . . . . . . . . 8

2.1 Burst (top figure) and background (bottom figure) selection for an SGR 1900+14 burst for spectral analysis. Red regions indicate parts included in spectral analysis. The dotted line in the top figure indicates the limit where detectors get paralyzed due to abundance of photon counts. Since 5 PCUs were active during this observation, the upper limit is 90000 counts/second. . . . . . . . . . . . . . . 15

3.1 Model curves for SGR1806-20 Burst Start Time (MET): 335364358.746 . . . . . . 34

3.2 Top panel: Fitted models for SGR1806-20 Burst Start Time (MET): 335364358.746. Lower group of panels: Fit residuals for the same event. The models for fit residuals are $\mathrm{BB}+\mathrm{BB}, \mathrm{BB}+\mathrm{PO}, \mathrm{COMPT}, \mathrm{LB}+\mathrm{LB}$ respectively from top to bottom. . . . . . .

3.3 SGR 1900+14 Fitted parameter distributions. The top two figures are the distributions of the photon index (left) and high energy cut (right) with a Gaussian fit (dashed lines) for the COMPT model. The two figures on the bottom are the distributions of the temperatures of the cold (left) and hot (right) additive blackbody components for the BB + BB model. . . . . . . . . . . . . . . . . .

3.4 SGR 1900+14 Fitted flux distributions for PCA (right) and HEXTE fits (left) for the COMPT (top) and BB+BB (bottom) models. Gaussian fits are shown with dashed lines. . . . . . . . . . . . . . . . . . . . . .

3.5 SGR 1900+14 Fitted fluence distributions for PCA (right) and HEXTE fits (left) for the COMPT (top) and BB+BB (bottom) models. Gaussian fits are shown with dashed lines. . . . . . . . . . . . . . . . . . . . . . .

3.6 SGR 1900+14 Log-log plots of total energy for PCA (right) and HEXTE fits (left) for the COMPT (top) and BB+BB (bottom) models. . . . . . . . . . . . . . . 
3.7 SGR 1806-20 Fitted parameter distributions. The top two figures are the distributions of the photon index (left) and high energy cut (right) with a Gaussian fit (dashed lines) for the COMPT model. The two figures on the bottom are the distributions of the temperatures of the cold (left) and hot (right) additive blackbody components for the $\mathrm{BB}+\mathrm{BB}$ model. . . . . . . . . . . . . . .

3.8 SGR 1806-20 Fitted flux distributions for PCA (right) and HEXTE fits (left) for the COMPT (top) and BB+BB (bottom) models.

3.9 SGR 1806-20 Fitted fluence distributions for PCA (right) and HEXTE fits (left) for the COMPT (top) and BB+BB (bottom) models. Gaussian fits are shown with dashed lines. . . . . . . . . . . . . . . . . . . . . . . . . .

3.10 SGR 1806-20 Log-log plots of total energy for PCA (right) and HEXTE fits (left) for the COMPT (top) and BB+BB (bottom) models. . . . . . . . . . . . . . . . . 46

3.11 SGR 1900+14 (left) and SGR 1806-20 (right) Peak Energy Distributions. . . . . . 65

4.1 Visual representation of resulting p-values. P-value above 0.9 indicates that COMPT model describes simulated spectra best. Bursts where LB+LB model was selected as the best describing model within thermal models are shown in red. . . . . . . . .

4.2 Seed Model $\chi^{2}$ - Test Model $\chi^{2}$ distributions for three SGR 1806-20 bursts. Blue shaded regions represent rejection regions of the seed (COMPT) model (where $\Delta \chi^{2}>3.84$ ) (a) $\Delta \chi^{2}=\chi_{C O M P T}^{2}-\chi_{B B+B B}^{2}$ for Burst ID: 328807661. (b) $\Delta \chi^{2}=$ $\chi_{C O M P T}^{2}-\chi_{B B+P O}^{2}$ for Burst ID: 168976265. (c) $\Delta \chi^{2}=\chi_{C O M P T}^{2}-\chi_{L B+L B}^{2}$ for Burst

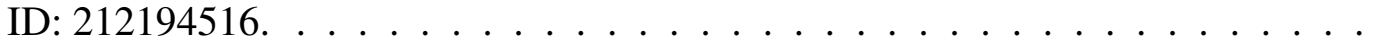

4.3 Upper left panel (a) SGR J1550-5418, SGR 1900+14, SGR 1806-20 emission area vs. hot and cold blackbody temperatures. $R^{2} \propto T^{-3}$ and $R^{2} \propto T^{-4}$ are drawn with dashed and solid lines respectively for comparison only. Upper right panel (b) SGR 1806-20 emission area vs. hot and cold blackbody temperatures grouped by total flux values with corresponding broken power law fits. Break index in kT space $(\mathrm{keV})$ are shown with color-coded arrows. Lower left panel (c) SGR J1550-5814 emission area vs. hot and cold blackbody temperatures with broken power law (red dashed line) and linear model (black dashed line) fits. Arrows represent break index in kT space (keV). Lower right panel (d) SGR 1900+14 emission area vs. hot and cold blackbody temperatures with broken power law fit shown in dashed line. Break index in $\mathrm{kT}$ space $(\mathrm{keV})$ is shown with the arrows. 


\section{List of Tables}

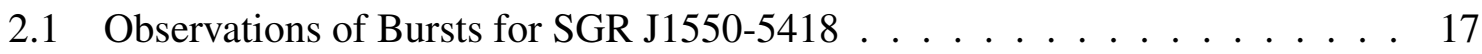

2.2 Observations of Bursts for SGR 1900+14 _ . . . . . . . . . . . . . . . . . 19

2.3 Observations of Bursts for SGR 1806-20 . . . . . . . . . . . . . . 24

3.1 Percentage of acceptable spectral fits based on $\chi^{2}$ probability for the given

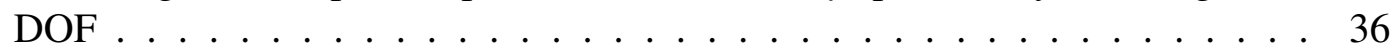

3.2 Spectral Properties of SGR J1550-5418 Bursts. . . . . . . . . . . . . . . 48

3.3 Spectral Properties of SGR 1900+14 Bursts. . . . . . . . . . . . . . . 50

3.4 Spectral Properties of SGR 1806-20 Bursts. . . . . . . . . . . . . . . 55

$4.1 \quad$ P-values . . . . . . . . . . . . . . . . . . . . . . 70

4.2 Broken Power Law Fit Results with Corresponding Flux Intervals _ . . . . 74 


\title{
List of Abbreviations
}

\author{
AXP Anomalous X-ray Pulsar \\ BB Blackbody Model \\ BB+BB Two Additive Blackbody Models \\ BB+PO Additive Blackbody and Power law Models \\ COMPT Comptonized Model with Cutoff Energy Parametrization \\ COMPT2 Comptonized Model with Peak Energy \\ GRB Gamma-ray Burst \\ LB Lyubarsky (Modified Blackbody) Model \\ LB+LB Two Additive Lyubarsky (Modified Blackbody) Models \\ MET Mission Elapsed Time \\ PCA Proportional Counter Array \\ PCU Proportional Counter Unit \\ RXTE Rossi X-ray Timing Explorer \\ SGR Soft Gamma Repeater
}




\section{Chapter 1}

\section{Introduction}

\subsection{Neutron Stars}

A neutron star is a compact object formed after a massive star completes its star-life: Ordinary stars remain stable as a result of the radiation pressure and gravitational pull balance. When a massive star runs out of its radiative energy source (nuclear fusion) the gravitational pull causes the star to collapse on itself, causing a supernova explosion. Neutron stars are formed after a supernova explosion when the degenerate pressure of fermions within the object are enough to keep it stable under gravity. The degenerate pressure arises from the extremely high densities $\left(\sim 10^{17} \mathrm{~kg} / \mathrm{m}^{3}\right)$ of neutron stars. Under such high densities, it was thought to become energetically favorable to turn most protons into neutrons, thereby causing the terminology 'neutron stars', although it is possible that more exotic phases (e.g. hyperons, boson condensates, quark matter) within a neutron star exist due to the extremely high densities.

\subsubsection{General Properties}

Neutron stars were first detected with unusual pulses in the radio band by Hewish \& Okoye 1965 although the existence of extremely dense objects were proposed over a decade ago by Landau 1932, soon after the discovery of the neutron (Chadwick 1932). Hewish et al. 1968 later classified the first known neutron stars causing these pulsations as radio pulsars. Since then, theoretical and observational knowledge of these objects has grown vastly, together with the number of neutron stars discovered. Initially discovered in the radio band, neutron stars are now known to be emitting radiation in the X-ray, $\gamma$-ray and in some cases optical band. With the help of improvements in $\mathrm{X}$-ray and $\gamma$-ray measurements, neutron stars with distinct characteristics have been discovered, causing different classifications within the neutron star family.

The distinct characteristics of classes of neutron stars are caused by their different temporal, 
spectral and dynamical properties. Neutron stars are found isolated and in binary systems (classified as LMXBs and HMXBs depending on the mass of the companion star). The pulsation periods of pulsars range from miliseconds to hundreds of seconds with magnetic field strength between $10^{8}-10^{15} \mathrm{G}$. In the low period limit are the milisecond pulsars with low magnetic fields, thought to be previous pulsars spun-up by accretion from a binary companion (Alpar et al. 1982).

Magnetic field strength of neutron stars also pose a method of classification. More recently it has been proposed that neutron stars in the high magnetic field limit ( $\sim 10^{15} G$ ) show distinct temporal and spectral properties (i.e. in spin, spin-down rate, and bursts). These types of neutron stars were first classified under the names "Anomalous X-ray Pulsars" (AXPs) and "Soft Gamma Repeaters" (SGRs). The first discovery of these sources date back to 1979 when repeated bursts from the source currently known as SGR 1900+14 with softer spectra than Gamma-ray Bursts (GRBs) were detected. (Mazets et al. 1979). Current research shows similar characteristics of AXPs and SGRs and they are thought to represent a common class of neutron stars called magnetars. (Kaspi $\&$ Beloborodov 2017). A more detailed description on magnetar properties are presented in Section 1.2 since we focus on these objects in our investigations.

\subsubsection{Structure and Equation of State}

Typically, neutron stars have mass $\sim 1.4 M_{\odot}$ and radii $\sim 10 \mathrm{~km}$. The currently known masses of $\sim$ 35 neutron stars are within the range 1.17 to $2.0 M_{\odot}$ (Özel \& Freire 2016). The radii of more than 12 neutron stars have been constrained to the range $9.9-11.2 \mathrm{~km}$. These measurements, especially the fact that the masses are higher than what degeneracy pressure alone could hold, support that repulsive nuclear forces also play a role to shape the neutron star structure. Therefore the densities of neutron stars must exceed nuclear saturation density, presumably by as high as 8 times. This means that relativistic effects should be taken into account to study the structure as well as the mass and radius of neutron stars.

Theoretically, there exists one equation of state for neutron stars that describes their global structure in terms of the pressure-energy density relation. Assuming a non-rotating spherical object in hydrostatic equilibrium and taking relativistic effects into account in general relativity, incorporating the correct equation of state (commonly decribed as pressure as a function of density or energy density) into the TOV (Tolman-Oppenheimer-Volkoff) equations would yield the global structure of the neutron stars. (Oppenheimer \& Volkoff 1939; Tolman 1939). However, the extreme compactness of neutron stars pose a difficulty to apply boundary conditions on mass and radius and solve such equations, since it is impossible to test the densities within the core under terrestrial conditions experimentally. As a result, numerous equations of state have been proposed with different boundary conditions which in turn empose different mass-radius relationships (see Figure 1.1).

In current theoretical work with field theory and QCD calculations on neutron star structure, 

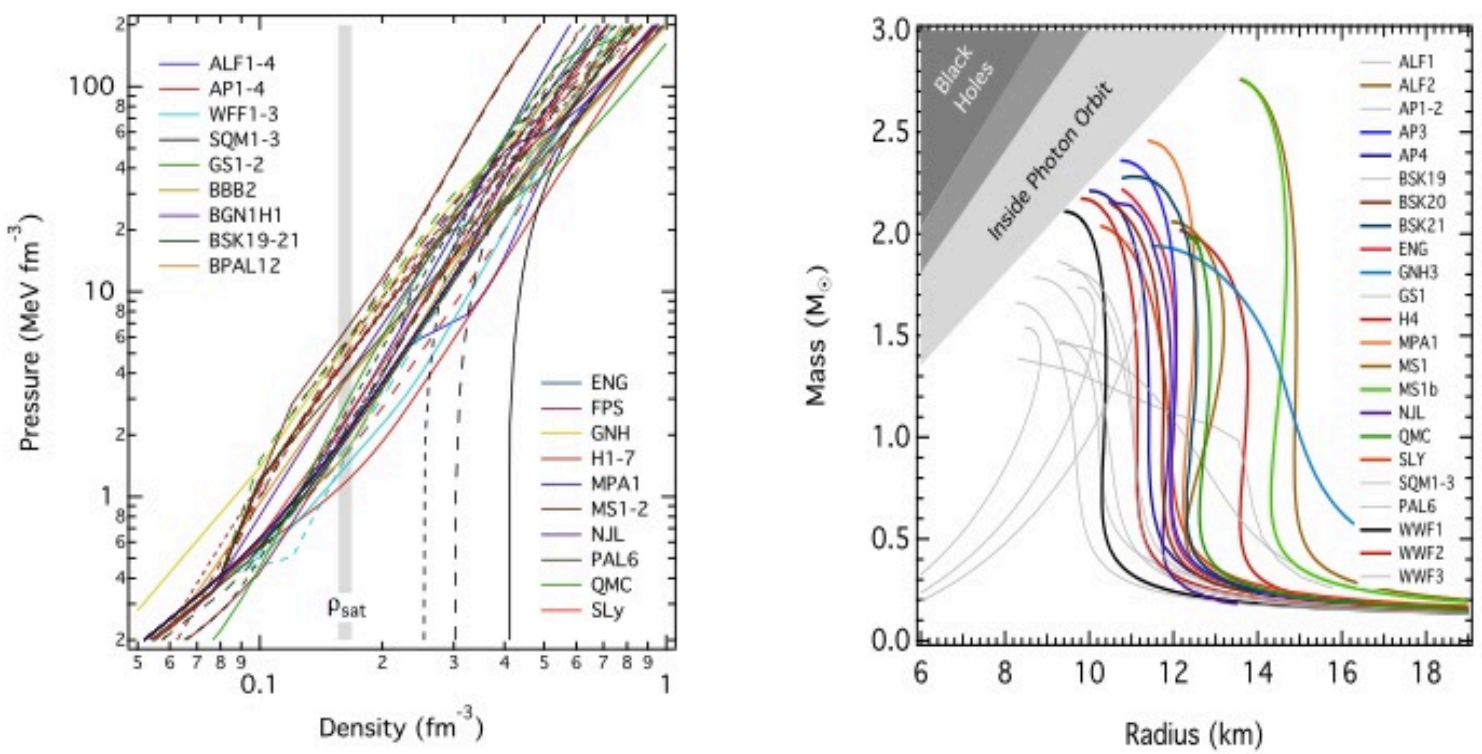

Figure 1.1 Left Examples of neutron star equations of state (pressure as a function of density) proposed under different assumptions (for hadronic matter and strange quark matter (SQM)) Right Mass and radius relationships inferred from different equations of states proposed. Figure is taken from Özel \& Freire 2016

the composition of the core remains an open question. Due to the extreme densities in the core, it is possible that instead of nucleons; hyperons carrying strange quarks, pion or kion condensates and/or quark matter exist in the core. This makes neutron stars perfect laboratories to study extremely dense that cannot otherwise be observed, as well as to test general relativity under strong fields.

\subsubsection{Cooling}

Theoretical and observational studies concerning neutron star temperature evolution may also provide information about states of matter under extreme densities. Neutron stars are though to be born with temperatures $\sim 10^{10} \mathrm{~K}$ and cool very rapidly due to dominant neutrino emission (although the star is observable in X-ray band) possibly by direct Urca or modified Urca processes (see Figure 1.2 for the cooling curve of a sample of neutron stars). Observational limits on surface temperatures using thermal emission models show a clear distinction between the upper limit on temperature of young pulsars (e.g. Crab Pulsar with $\mathrm{t}=1 \mathrm{kyr}, \mathrm{T}<2 \mathrm{MK}$, Weisskopf et al. 2004; PSR J0205+6449 with $\mathrm{t}=0.82 \mathrm{kyr}, \mathrm{T}<1.1 \mathrm{MK}$, Slane et al. 2002) and older pulsars (e.g. RX J0720.4-3125 with $\mathrm{t}=1300$ kyr, $\mathrm{T}<0.5$ MK, Motch et al. 2003; see Yakovlev \& Pethick 2004 for a more detailed review) 


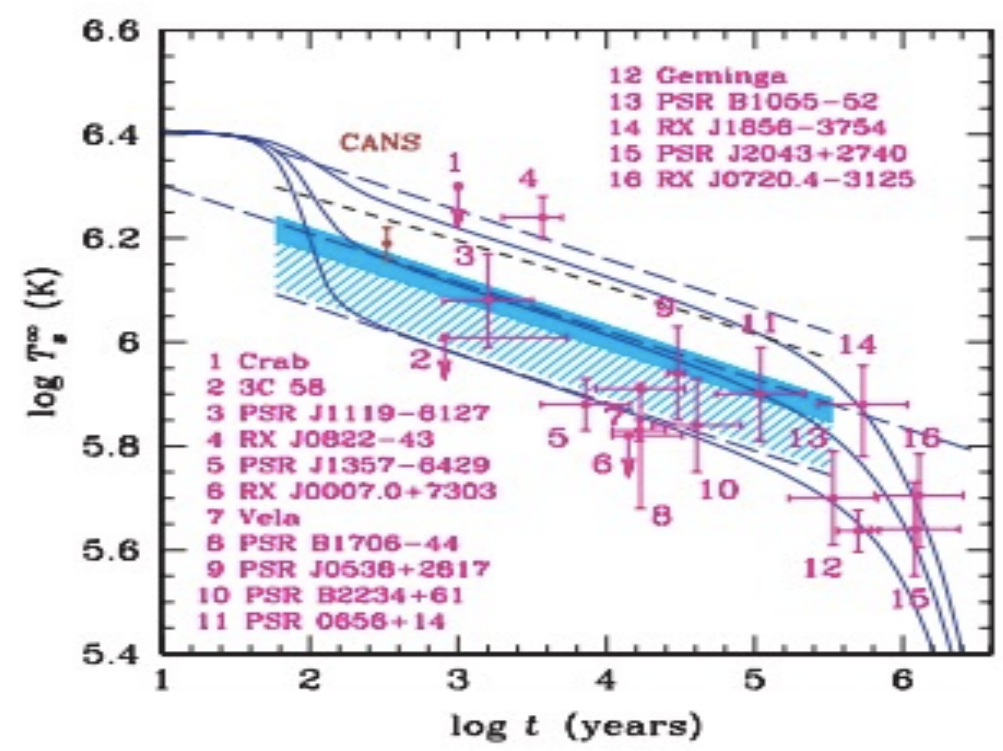

Figure 1.2 Cooling curve (log Temperature (K) vs log time (yrs)) of a sample of neutron stars. Figure is taken from Yakovlev et. al. (2011)

\subsubsection{Limitations on Mass}

As a result of the uncertainties in matter phases, the correct equation of state of a neutron star is currently unknown. However, studies with different assumptions lead to different equations of state to enforce limits on mass. It is plausible that an upper limit on mass exists, since when the degeneracy pressure cannot hold the neutron star it would become a black hole. First measurements by Oppenheimer \& Volkoff 1939 using non-interacting degenerate relativistic neutron gas equation of state yielded of mass of $0.7 M_{\odot}$ although we now know that much more massive neutron stars exist. The estimates on maximum mass of neutron stars go as high as $\sim 3.2 M_{\odot}$ (Rhoades \& Ruffini 1974, Lattimer 2012) in extreme cases. However, model predictions by van Kerkwijk et al. 2011 suggest a narrower range of $2.4 \pm 0.12 M_{\odot}$. Similarly, observational studies of Webb \& Barret 2007 set an upper limit of $\sim 2.4 M_{\odot}$.

\subsubsection{Temporal Characteristics}

Most radio pulsars are thought to be born with periods in the order of miliseconds, although periods go up to $\sim 12 \mathrm{~s}$. Many models for longer period pulsars propose spin-down due to accretion from a companion star (e.g. see Illarionov \& Kompaneets 1990, Ikhsanov 2007 for a theoretical viewpoint and Hartman et al. 2009 for a supporting observational study). Spins show a decrease with $\dot{P}$ in the range $\sim 10^{-21}$ to $10^{-10} s s^{-1}$ (Manchester et al. 2005). P and $\dot{P}$ measurements give idea about the correct age and magnetic field strengths of pulsars. 


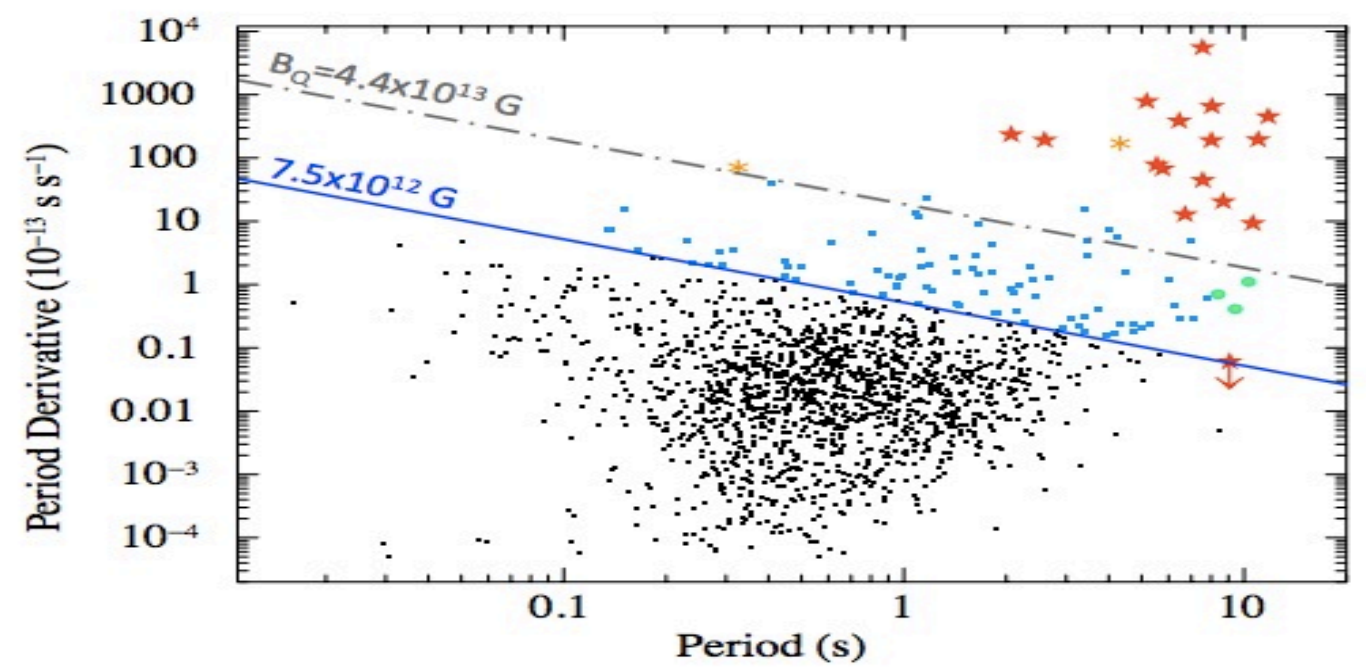

Figure $1.3 \dot{P}$ vs. P plot of neutron stars. Squares represent pulsars colored by magnetic field strength. Red stars indicate magnetars separated by the grey solid line indicating magnetic field above $4.4 \times 10^{13} \mathrm{G}$. Figure is taken from Rea et. al. (2010)

Although $\dot{P}$ shows a steady increase in pulsars, sudden spin-ups (glitches) and more rarely sudden spin-downs (anti-glitches) (see e.g. Şaşmaz Muş et al. 2014) from pulsars have been observed. Glitches typically have $\Delta P / P$ in the range $10^{-9}$ to $10^{-5}$ in radio pulsars and magnetars. Pulsars and magnetars also show long-term changes in $\dot{P}$ ( $\dot{P}$ being usually positive and $\Delta \dot{P} / \dot{P}$ up to $\mathrm{O}(0.01)$.). Radio pulsars typically recover up to 0.5 of the glitch within $\sim 1$ week, whereas magnetars show much faster glitch recovery, recovering the full glitch and in some cases even more such that the overall effect is a spin-down. (Kaspi \& Beloborodov 2017)

\subsection{Magnetars}

Magnetars are young neutron stars with high inferred magnetic fields $\left(\sim 10^{14} G\right)$ that have characteristic repeated emissions in the hard X-ray and soft $\gamma$-ray bands with high luminosities (spanning $10^{30}-2 \times 10^{35} \mathrm{ergs} / \mathrm{s}$ in the $2-10 \mathrm{keV}$ band in quiescent state (Kaspi \& Beloborodov 2017). This repetitive radiative behaviour in the hard X-ray and soft $\gamma$-ray bands differ from classical Gammaray bursts (GRBs) and therefore these sources were first regarded as unusual and classified under the names Anomalous X-Ray Pulsars (AXPs) and Soft Gamma Repeaters (SGRs). Currently, AXPs and SGRs are thought to belong to similar class of neutron stars called magnetars due to their similar characteristics including repeated bursts and outbursts observed from many AXPs. AXP bursts show similar spectral shapes to SGR bursts, although SGR bursts generally have relatively harder 


\section{X-ray spectra.}

Magnetars have radiative behiavior that is considered unusual in the pulsar regime. That is, their high X-ray luminosities cannot be explained by rotational energy loss or accretion from a companion since no current evidence of binary companions exist. They are thought to be younger systems with longer periods $\sim 2-12 s$ and spin down rates with $(P / \dot{P} \sim$ a few thousand years). Their high spin-down rate and longer periods differ significantly from pulsars and imply a high magnetic field strength with $B>10^{14} \mathrm{G}$ for the majority of these sources (See Figure 1.3). There are currently 29 known magnetars ( 23 confirmed +6 magnetar candidates) ( Kaspi \& Beloborodov 2017). Since magnetars are generally discovered only when they go under repeated burst episodes, it is likely that the known magnetars and magnetar candidates represent a small fraction of the whole magnetar population. All current known magnetars are strictly confined to the Galactic Plane, at scale heights $(\sim 20-30 \mathrm{pc})$ much smaller than the radio pulsars, suggesting they are very young sources $\left(<10^{5} \mathrm{yrs}\right.$ of age). In accordance with the youth of magnetars inferred from their spatial distributions and spin-down rates, a large number of magnetars are associated with supernova remnants. More detailed reviews on magnetars are discussed in Mereghetti et al. 2015 and more recently in Kaspi \& Beloborodov 2017

\subsubsection{Theoretical Models}

Four main models currently exist to explain the unusual behaviour of these sources, the oldest and most widely-accepted being the magnetar model proposed by Thompson \& Duncan 1995. In the magnetar model, the variety of energetic magnetar emission mechanisms are thought to be governed by the decay of their extremely strong magnetic fields $\left(B \sim 10^{14}-10^{15}\right) \mathrm{G}$. Alternatively, in the fallback disk model, the star is thought to be of conventional magnetic fields and powered by the energy of mass inflow of accreted mater from a fallback disk that forms during the supernova explosion. ( Chatterjee et al. 2000; Alpar 1999; Alpar 2001). Several other models to explain magnetarlike behaviour also exist, such as the quark star model where the star is thought to be formed by pure quark matter (Xu 2007) and models suggesting AXPs and SGRs are types of massive white dwarfs with high magnetic fields and spin (Paczynski 1990). Here, our coverage on theoretical models on magnetar behaviour will focus on the magnetar model and the fallback disk model. Emission of energetic hard X-ray bursts remains the most characteristic signature of magnetar-like behaviour. Observational studies on X-ray bursts promise insight into emission mechanisms as well as structural properties of magnetars. 


\subsubsection{Magnetar Model}

In the magnetar viewpoint, when a new-born neutron star has fast enough spin (in the milisecond range), convection forces caused by entropy act as an efficient helical dynamo to produce extremely strong $\left(\mathrm{B} \sim 10^{14}-10^{15} \mathrm{G}\right.$ ) magnetic fields exceeding the quantum critical value

$$
B_{q c} \equiv m_{e}^{2} c^{2} / \hbar e \approx 4.4 \times 10^{13} G
$$

where energy difference of Landau levels of electrons equal their rest mass energy. This causes the main difference between pulsars and magnetars. That is, magnetic field strength begins to dominate the rotational energy of magnetars due to the dynamo effect at a very young age. The energetic bursts emitted from magnetars are thought to be triggered when the built-up magnetic field stress causes fracturing of the crust. This mechanism as well as the observed bursts differ from pulsars since the dipole field of a magnetar is strong enough to dominate the crustal deformation energy. Therefore, magnetic stress causes fractures in the crust when ambipolar diffusion and Hall drift effects cause the magnetic field to drift away from equilibrium. The fractures in the magnetrar crust causes instabilities that displaces magnetic field lines, causing Alfvén waves. This mechanism can power the short bursts as well as cause the observed spin-down rates of magnetars. (Thompson \& Duncan 1995). The quick damping of such instabilities that lead to displacements (i.e. twisting) of the magnetic field lines are thought to create "fireballs" embedded in an optically thick corona of electron-positron pairs as shown in Figure 1.2. The discusssed $e^{ \pm}$pairs in the corona, or alternatively photon splitting, may account for the Comptonization processes (inverse Compton upscattering of emitted photons) that can cause the tails in the higher energy range of the otherwise thermal observed spectra of magnetar bursts (Thompson \& Duncan 2001). In the magnetar model, the mentioned crustal fractures as well as larger displacements in the magnetic field lines due to rearrangements in the stronger core magnetic field eventually causes reconnection events that trigger the much more energetic giant flares observed from magnetars.

\subsubsection{Fallback Disk Model}

Alternatively, the radiative behaviour of AXPs and SGRs are explained in the fallback disk model (Alpar 1999; Chatterjee \& Hernquist 2000; Alpar 2001) as resulting from accretion from a fossil disk that forms as a result of matter falling back onto the newborn neutron star following the collapse of the progenitor. In the fallback disk model, the accretion from the fallback disk is thought to power emission from AXPs and SGRs which have conventional dipole magnetic field strengths $\left(\sim 10^{12} G\right)$. The accretion disk may exists only above the radius of the magnetosphere, which is $R_{m}$ $\approx 0.5 r_{A}$, where $r_{A}$ is the Alfvén Radius (turnover point at which magnetic field starts dominating the accretion). Inside the Alfvén Radius, matter accretes onto the star following the magnetic field 


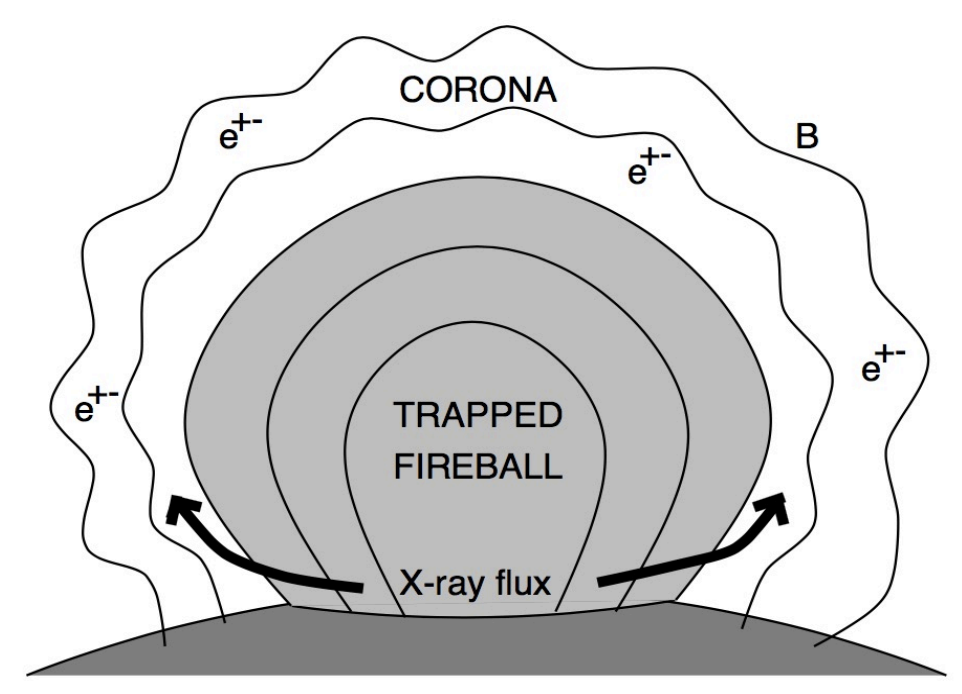

Figure 1.4 Depiction of the trapped fireball in a corona of electron-positron pairs. Figure is taken from Thompson \& Duncan 2001

lines since magnetic field strength of the order $10^{12} \mathrm{G}$ dominates accretion. Depending on the position of $R_{m}$ with respect to the light cylinder radius and the corotation radius, accretion may or may not be permitted even though a fallback disk forms in a neutron star (since above the light cylinder radius the disk will not interact with the star). As a result, this model suggests that a neutron star may behave as a radio pulsar or an accreting object for part of its life independently of whether a fallback disk forms or not. (Chatterjee \& Hernquist 2000).

In the fallback disk model, there are three main stages that a magnetar may go throughout its life: propeller phase, tracking phase and ADAF phase. The magnetar is expected to be luminous in the X-ray band in the tracking phase and dim in the other two phases. A magnetar may skip the tracking phase during its formation and start its evolution in the ADAF phase and therefore be never observed as a bright $\mathrm{X}$-ray source. The propeller phase is when $R_{m} \gg R_{c}$, where $R_{c}$ is the corotation radius (i.e. when the star spins much faster than the disk at $R_{m}$ ). At this phase, centrifugal forces will cause the accreting matter to be pushed out before reaching the surface. Therefore, accretion will be inefficient. Since accretion is inefficient, the neutron star will be much more dim in the X-ray band in the propeller phase. Although matter can not reach the surface, it will still reach $R_{m}$ where it can cause the star to spin-down rapidly due to angular momentum transfer. As the rotation rate of the star and the disk (at $\left.R_{m}\right)$ become similar, the system will enter the tracking phase. In this phase, accretion is effective and therefore the star is expected to be bright in the X-ray band. However, the accreting magnetar will be in a quasi-static equilibrium since the accretion rate declines steadily with time as in

$$
\dot{m}=\dot{m}_{0}(t / T)^{-\alpha}
$$


where $\dot{m}$ is the mass accretion rate and $\dot{m}_{0}$ is a constant, as opposed to the steady accretion rate in LMXBs. As accretion rate decreases with time, the object will enter the ADAF (advectiondominated accretion flow) phase where it is faint in the X-ray band again. The fact that these sources are bright only in the tracking phase when their periods are close to equilibrium may account for the narrow observed period ranges of these sources.

In terms of their energetic emissions, Thompson et al. 2000 argued that high luminosity giant flares would cause high radiation momentum that would force the accretion disk away from the neutron star and the disk would take months to years to return to its original position. Therefore, Thompson et. al. propose a fallback disk would not account for the decay and persistent X-ray emission after a giant flare occurs (e.g. the X-ray Emission of SGR 1900+14 following the August 27th Giant Flare). On the other hand, Ertan \& Alpar 2003 proposed with numerical simulation results that while the inner parts of the disk get pushed out due to radiation momentum during giant flares, matter remains bound to the system and that relaxation of the disk may power more accretion, which would account for the enhanced X-ray emission and decay after the giant flares.

It is important to note that whether the mechanisms leading to radiative behaviour of magnetars is magnetospheric, crustal, due to a debris disk or due to more exotic phases of matter within the star (e.g. quark matter) remains unresolved.

\subsubsection{Bursts}

Magnetars show three types of transient radiative behaviour: bursts, outbursts and giant flares. Burtsts are short-lived events (lasting a few miliseconds to seconds) of flux increase and in some cases are followed by a tail of afterglow. Outburtsts occur suddenly but last much longer (weeks to months), typically consisting of multiple shorter bursts. Outbursts are followed by longer tails, lasting for months. Giant flares are the most luminous of the types of sudden increase in flux (releasing over $10^{44}$ ergs of energy), and are usually described as catastrophic events for magnetars. Here, the discussion will be concentrated on bursts, the shortest-lived transient radiative magnetar activities. Flux may also peak a single time or multiple times during radiative activity. The analysis covered here will be based on single peak bursts.

The magnetar population known today consists of 29 sources, 18 of which (whose spin has also been measured) emitted short duration (lasting only a fraction of a second) but very luminous bursts (Turolla et al. 2015). Magnetar bursts occur sporadically on random occasions, and the total number of bursts varies from a few to hundreds during the burst active episode of the underlying magnetar. Each burst has the potential of revealing new insights into the burst triggering and radiation emission mechanisms. The principle ingredient of magnetar bursts is a type of disturbance by the extremely strong magnetic fields. According to the magnetar model, the solid crust of a neutron 
star could fracture when extremely large magnetic pressure builds-up on it (Thompson \& Duncan 1995) In this view, the scale of burst energetics would be related to the size of fractured crustal site. Thompson et al. 2002a suggested that the magnetospheres of these objects are globally twisted. As an alternative burst trigger mechanism, Lyutikov 2003 proposed that magnetic reconnection might take place in the twisted magnetosphere of magnetars. It is important to note that whether the trigger for short magnetar bursts is crustal or magnetospheric is still unresolved.

\subsubsection{Emission Mechanisms}

The observed bursts are the end products of their initial triggers. Therefore, emission radiated away as burst might not be the direct consequence of the ignition, but a number of processes in between are likely involved. In the magnetar view, the trigger mechanism leads to the formation of a trapped fireball in the magnetosphere, composed of $\mathrm{e}^{ \pm}$-pairs as well as photons (Thompson $\&$ Duncan 1995). Bursts are due to radiation from these trapped pair rich fireballs. Additionally, emerging radiation is expected to be modified as it propagates through strongly magnetized and highly twisted magnetosphere (Lyubarsky 2002). It is, therefore not straightforward to unfold the underlying mechanism from the burst data.

Spectral and temporal studies on magnetar bursts are still the most important probes to help distinguish mechanisms that could modify the emerging radiation of bursts.i In recent spectral investigations, both thermal and non-thermal scenarios were invoked (e.g. Feroci et al. 2004, Israel et al. 2008, Lin et al. 2012, van der Horst et al. 2012). In the non-thermal viewpoint (often analytically expressed with a power law with an exponential cutoff), the photons emerging from the ignition region are repeatedly Compton up-scattered by the $\mathrm{e}^{ \pm}$-pairs present in the magnetosphere. The corona of hot electrons may emerge in the inner dynamic magnetosphere due to field line twisting (Thompson et al. 2002b, Thompson \& Beloborodov 2005, Beloborodov \& Thompson 2007). The density and optical thickness of the corona, as well as the electron temperature set a spectral turnover. Consequently, the peak energy parameter of the Comptonized (often labeled as COMPT) model is interpreted in relation to the electron temperature. Time integrated spectra of nearly 300 bursts from SGR J1550-5418 result in an average power law photon index of 0.92, and cutoff energy $\left(\mathrm{E}_{\text {peak }}\right)$ is typically around $40 \mathrm{keV}$ (van der Horst et al. 2012).

The alternative approach to interpret magnetar burst spectra is the thermal emission due to a short-lasting thermal equilibrium of electron-photon pairs, usually described with the sum of two blackbody functions (see e.g., Feroci et al. 2004, van der Horst et al. 2012). This dual blackbody scheme approximates a continuum temperature gradient due to the total energy dissipation of photons throughout the magnetosphere. The coronae are expected to be hotter at low altitudes than the outer layers. Therefore, the coronal structure suggests that the high temperature blackbody com- 
ponent be associated with a smaller volume than the cold component. Recent studies of magnetar burst spectra with the two blackbody model yields 3-4 keV and 10-15 keV for the temperature of cold and hot blackbodies, respectively (e.g. Olive et al. 2003, Feroci et al. 2004, Lin et al. 2012, van der Horst et al. 2012).

\subsubsection{Overview of Broadband Spectral Bursts Analyses}

In the past, spectral Studies of SGR bursts have usually been conducted using individual instruments on energy ranges above or below $\sim 15-20 \mathrm{keV}$, but not both. For example, Israel et al. 2008 reported that a single blackbody function with temperature $\sim 10 \mathrm{keV}$ describe BAT burst spectra well. The spectral investigations usually focus on the Comptonized model, Bremsstrahlung (OTTB), a sum of two blackbody functions $(\mathrm{BB}+\mathrm{BB})$ and a sum of blackbody and power law functions $(\mathrm{BB}+\mathrm{PO})$. However, it was recently shown that the actual spectral nature of these bursts can be conclusively determined if the spectral analysis is performed on a wide energy coverage. Several studies indicate that on such broad energy coverages, complex models (e.g. BB+BB, Comptonized) describe spectra better than simple power law or blackbody models. (e.g., Feroci et al. 2004; van der Horst et al. 2012) It was also shown on multiple studies that bremsstrahlung model in a spectral range coverage down to $1-2 \mathrm{keV}$ tends to overestimate flux at lower energies (e.g., Fenimore et al. 1994; Feroci et al. 2004).

More recently, Lin et al. 2012 and van der Horst et al. 2012 have conducted spectral analysis of SGR J1550-5418 with wider energy coverages. Van der Horst et al. used an energy range of 8-200 keV on GBM data with Comptonized and BB+BB models and found that the models fit the spectra equally well. Lin et al. have used XRT and GBM data on BB+BB and Comptonized models with a wider energy coverage of $0.5-200 \mathrm{keV}$ and found an average photon index of $-.58 \pm .09$ and average temperatures of $4.4 \pm 0.2$ and $16 \pm 0.4$ for the cold and hot blackbodies respectively. They also reported that on average, $\mathrm{BB}+\mathrm{BB}$ model better fits the spectra, indicating for the first time that the bursts might have a thermal character.

Studies on SGR $1900+14$ generally focus on BB+BB, BB+PO and Comptonized models for energies above $\sim 1.5 \mathrm{keV}$ and below $150 \mathrm{keV}$ with Nakagawa et al. 2007 being the exception (6-400 keV and 2-25 keV). Olive et al. 2003 have conducted spectral analysis on 1.5-100 keV on FREGATE data using BB+BB model only and found average BB temperatures to be $4.3 \mathrm{keV}$ and $9.8 \mathrm{keV}$. Feroci et al. 2004 worked on an energy coverage of 1.5-100 keV on SGR 1900+14 data observed with BeppoSAX. They have used BB+BB and Comptonized models and found an average

peak energy of $15.8 \pm 2.3$ and average temperatures for the cold and hot blackbody components to be $3.23 \pm 0.56$ and $9.65 \pm 0.95 \mathrm{keV}$.

Recent studies on SGR 1806-20 are relatively rarer than the other sources in this study. Nakagawa et al. 2007 studied bursts from SGR 1900+14 and SGR 1806-20 data observed with WXM 
and FREGATE , covering 2-25 keV and 6-400 keV respectively. They have found that OTTB fits gave poor results in many cases and used the Comptonized model instead, which can be regarded as an extension of the simple OTTB. They conclude that their data does not provide enough statistics to distinguish between $\mathrm{BB}+\mathrm{BB}, \mathrm{BB}+\mathrm{PO}$ and Comptonized models. Esposito et al. 2007 have studied SGR 1806-20 burst data taken with Suzaku and XMM-Newton instruments. Their widest energy coverage is $2-100 \mathrm{keV}$ with $\mathrm{BB}+\mathrm{PO}$ and $\mathrm{BB}+\mathrm{OTTB}$ models giving equally well fits.

\subsection{Thesis Outline}

In this thesis, we present the results of our systematic spectral analysis of a total of 388 bursts observed from three magnetars; SGR J1550-5418, SGR 1900+14 and SGR 1806-20, detected with the Rossi X-ray Timing Explorer (RXTE) between 2002 and 2009. We employed data collected with both instruments on board RXTE jointly, therefore performed our investigations in a broad energy range of 2-250 keV, which is the widest energy coverage for SGR 1806-20 bursts. We also modeled the time integrated burst spectra with the sum of two modified blackbody model (Lyubarsky 2002) for the first time. In Chapter 2, the data collection instruments and methodology for burst spectra generation are explained. The spectral analysis methodology and resulting parameter values and distributions will be given in Chapter 3. In Chapter 4, implications of analysis results will be discussed together with simulation results in terms of comparison between model fitting powers. We also show that our results indicate a significant correlation between effective radiation area and blackbody temperatures in Chapter 4 and discuss the relationship between the two in different flux ranges. 


\section{Chapter 2}

\section{Observations and Burst Sample}

\subsection{Instruments}

For our spectral investigations, we used data collected with the Rossi X-ray Timing Explorer (RXTE) mission built by NASA Goddard Space Flight Center, which was operational over $~ 16$ years from December 1995 until the end of 2011. The mission carried three instruments: Proportional Counter Array (PCA), High Energy X-ray Timing Explorer (HEXTE) and All Sky Monitor (ASM). This thesis focuses on the observations of PCA and HEXTE. PCA and HEXTE are coaligned with the same view but operate in different energy ranges so that a broadband energy range analysis using data collected from the two instruments (between 2-250 keV) is possible with an overlap between 15-60 keV. A more detailed technical description of instruments on board are given on the Technical RXTE Appendix ${ }^{1}$ and descriptions on data reduction and analysis are given in the RXTE Cookbook ${ }^{2}$.

PCA operated between $2-60 \mathrm{keV}$ energy range and consisted of an array of five sealed xenon $(90 \%)$ and methane (10\%) filled multi-anode proportional counter units (PCUs). Each unit has a collecting area of $1600 \mathrm{~cm}^{2}$ and was optimally sensitive in the energy range of $2-30 \mathrm{keV}$ (Jahoda et al. 2006). PCUs are nearly identical and operate independently of each other. Magnetar burst data collected with PCA provides medium energy resolution (64 or 256 energy channels) and a superb time resolution of $1 \mu \mathrm{s}$. The efficiency is higher and the residual background event rate is much lower for PCA at lower energies.

HEXTE consisted of two clusters each containing four NaI/CsI scintillation counters. The increased sensitivity of HEXTE in higher energy ranges is attained by the large collective areas of counters. The net open area of each counter is $\sim 225 \mathrm{~cm}^{2}$, making the total collective area of one cluster $\sim 800 \mathrm{~cm}^{2}$. HEXTE operates in the energy range $15-250 \mathrm{keV}$ with a time resolu-

\footnotetext{
${ }^{1}$ https://heasarc.gsfc.nasa.gov/docs/xte/RXTE_tech_append.pdf

${ }^{2}$ https://heasarc.gsfc.nasa.gov/docs/xte/recipes/cook_book.html
} 
tion of $8 \mu \mathrm{s}$. The two clusters have a "rocking" mechanism in which it rotates on its own axis, allowing for background measurements 1.5 or 3.0 degrees away from the source every 16 to $128 \mathrm{~s}$ simultaneously.

Photons interacting with RXTE are detected together with arrival-time and energy information. However, for brighter sources (i.e. bright bursts that exceed 20,000 counts/sec/pcu), only a fraction of this information is kept (also referred to as over-saturation).

Throughout its mission, RXTE observed magnetars at many occasions, substantially during their burst active phases: Bursts from SGR J1550-5418 were sampled from pointed RXTE observations that were performed between Oct 2000 - Apr 2010. SGR 1806-20 bursts were observed during its length burst active episode in 2003-2004, prior to the 2004 December 27 giant flare. SGR 1900+14 bursts were among 432 RXTE observations between Jun 1998 - Apr 2006.

\subsection{Generation of Spectra}

In a companion investigation of the temporal properties of magnetar bursts (Sasmaz Mus et al. in preparation), we performed a two-step burst identification scheme from these three magnetars using RXTE/PCA observations. We first employed a signal-to-noise ratio analysis to crudely identify the time of events, then applied a Bayesian blocks algorithm for final identification and morphological characterization of bursts (Sasmaz Mus et al., in preparation). We identified 179, 432, and 924 bursts from SGR J1550-5418, SGR 1900+14, SGR 1806-20, and respectively. Note that some bursts were very weak, consisting of only $\sim 10$ counts. We have first examined spectra of these bursts at varying intensities, and concluded that we would need at least 80 burst counts (after background subtraction) in order to constrain crucial spectral parameters at a statistically acceptable level. Hence, our burst sample for spectral analysis contain 42, 125, 221 bursts from SGR J15505418, SGR 1806-20, and SGR 1900+14 (see Table 2.1, 2.2, and 2.3), respectively.

We determined the time intervals for burst and background spectral integration using PCA observations as follows. For each burst, we first generated a light curve in the $2-30 \mathrm{keV}$ band with $0.125 \mathrm{~s}$ resolution spanning from $100 \mathrm{~s}$ before the peak time till $100 \mathrm{~s}$ after. We defined two nominal background extraction intervals; from 80 to $5 \mathrm{~s}$ before the burst, and from 5 to $80 \mathrm{~s}$ in the post burst episode. We excluded the time intervals of other short bursts from the background spectral integration (see the bottom panel of Figure 2.1). We then generated a finer light curve ( $2 \mathrm{~ms}$ resolution) in the same energy interval and selected the time interval of burst spectral integration. In this case, we excluded the time interval(s) during which the count rate exceeds 18000 counts/s/PCU in order to avoid any pulse pile-up related spectral biases (Figure 2.1, top panel).

We also eliminated bursts when one of the two HEXTE clusters was in 'rocking mode', switching to a different direction to obtain background emission. As a result, we were left with burst 

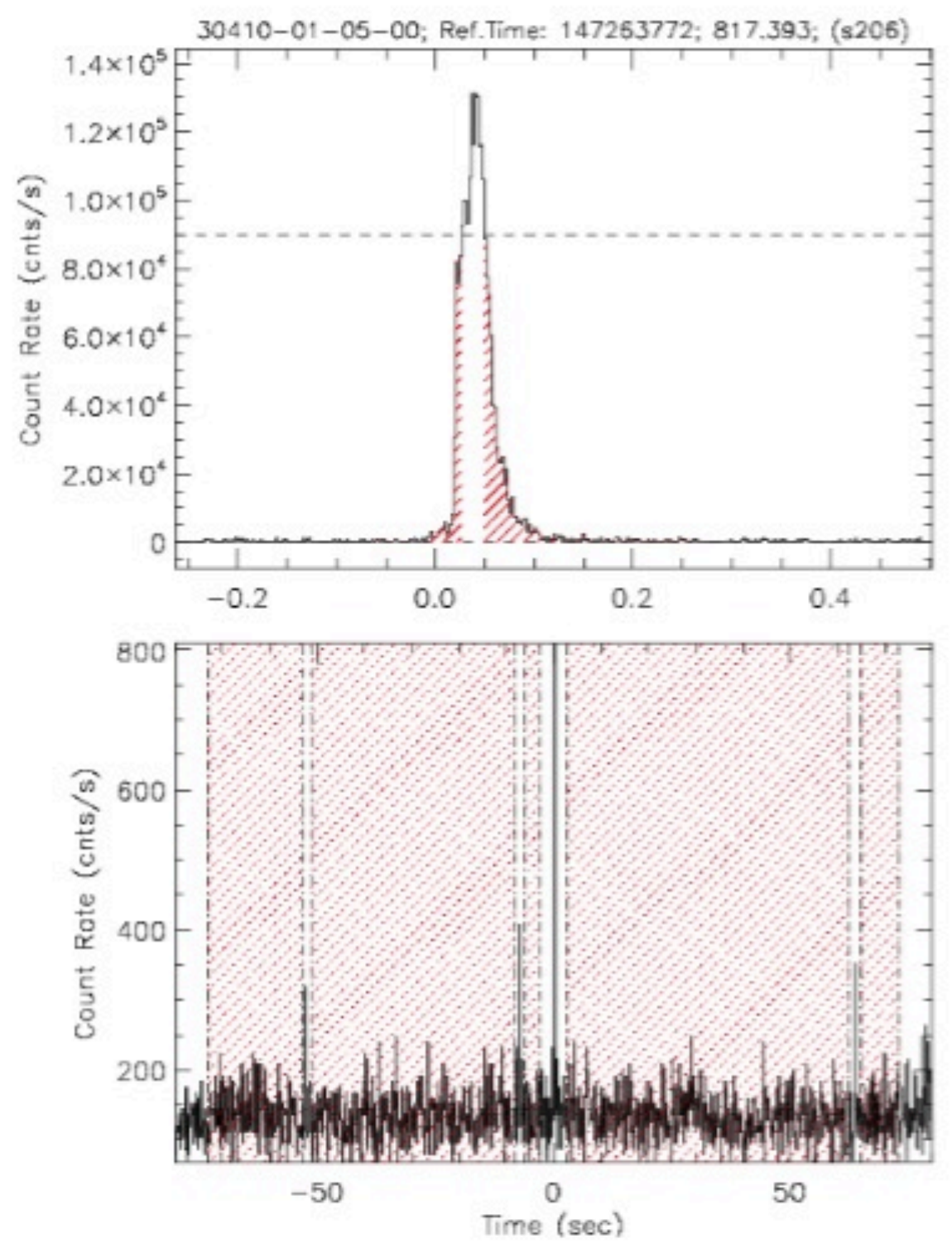

Figure 2.1 Burst (top figure) and background (bottom figure) selection for an SGR 1900+14 burst for spectral analysis. Red regions indicate parts included in spectral analysis. The dotted line in the top figure indicates the limit where detectors get paralyzed due to abundance of photon counts. Since 5 PCUs were active during this observation, the upper limit is 90000 counts/second.

data obtained when HEXTE was directed towards the source only. Therefore, we used the time intervals obtained from our PCA data analysis to generate HEXTE source and background spectra. We also took into account the malfunction that occurred in one of the detectors in cluster B during our analysis. We combined the spectra obtained from cluster A and cluster B. When only one of the clusters were operating during an observation, we obtained spectra using data collected with that particular cluster only. Finally, we grouped the spectra of PCA and HEXTE to minimum of 20 
burst count bins.

As a result of the abovementioned eliminations, the final numbers of bursts included in our spectral analysis are: 42 for SGR J1550-5418, 125 for SGR 1900+14 and 221 for SGR 1806-20. We list these bursts in Table 2.1 for SGR J1550-5418, Table 2.2 for SGR1900+14 and Table 2.3 SGR 1806-20. In the first column of these tables are the Bursts IDs in accordance with the spectral analysis result tables that will be later presented (Tables 3.1, 3.2 and 3.3). We list the starting time in MET in the second column and durations of the bursts $\left(T_{\text {Bayes }}\right)$ in seconds as obtained using a Bayesian blocks algorithm provided in Scargle et al. 2013 and the procedure discussed in Lin et al. 2013 in the third column. The Observation IDs from RXTE archives for the bursts are given in the fourth column. 
Table 2.1. Observations of Bursts for SGR J1550-5418

\begin{tabular}{cccc}
\hline \hline $\begin{array}{c}\text { Burst } \\
\text { ID }\end{array}$ & $\begin{array}{c}\text { Start time } \\
\text { in MET }\end{array}$ & $\begin{array}{c}\mathrm{T}_{\text {Bayes }} \\
\mathrm{s}\end{array}$ & ObsID \\
& & & \\
\hline 1 & 475274776.845 & 0.107 & $93017-10-17-00$ \\
2 & 475275167.175 & 0.254 & $93017-10-17-00$ \\
3 & 475276110.459 & 0.143 & $93017-10-17-00$ \\
4 & 475276179.299 & 0.000 & $93017-10-17-00$ \\
5 & 475276430.580 & 0.000 & $93017-10-17-00$ \\
6 & 475276565.382 & 0.189 & $93017-10-17-00$ \\
7 & 475277469.892 & 0.000 & $93017-10-17-00$ \\
8 & 475277658.503 & 0.125 & $93017-10-17-00$ \\
9 & 475280775.498 & 0.066 & $93017-10-17-00$ \\
10 & 475281285.917 & 0.256 & $93017-10-17-00$ \\
11 & 475282696.267 & 0.000 & $93017-10-17-00$ \\
12 & 475360594.236 & 0.105 & $93017-10-16-00$ \\
13 & 475360938.964 & 0.234 & $93017-10-16-00$ \\
14 & 475446854.015 & 0.500 & $93017-10-16-05$ \\
15 & 475449518.024 & 0.000 & $93017-10-16-05$ \\
16 & 475450021.046 & 0.564 & $93017-10-16-05$ \\
17 & 475451254.646 & 0.121 & $93017-10-16-05$ \\
18 & 475451316.638 & 0.037 & $93017-10-16-05$ \\
19 & 475451333.412 & 0.074 & $93017-10-16-05$ \\
20 & 475456857.203 & 0.174 & $93017-10-16-05$ \\
21 & 475458543.312 & 0.162 & $93017-10-16-05$ \\
22 & 475466911.865 & 0.555 & $93017-10-16-05$ \\
23 & 475468936.554 & 0.533 & $93017-10-16-05$ \\
24 & 475529885.755 & 0.213 & $94017-09-01-00$ \\
25 & 475529967.482 & 0.154 & $94017-09-01-00$ \\
26 & 475537351.812 & 2.996 & $94017-09-01-00$ \\
27 & 475560037.619 & 0.076 & $94017-09-01-02$
\end{tabular}


Table 2.1 (cont'd)

\begin{tabular}{cccc}
\hline \hline $\begin{array}{c}\text { Burst } \\
\text { ID }\end{array}$ & $\begin{array}{c}\text { Start time } \\
\text { in MET }\end{array}$ & $\begin{array}{c}\mathrm{T}_{\text {Bayes }} \\
\mathrm{s}\end{array}$ & ObsID \\
& & & \\
\hline 28 & 475807758.255 & 0.814 & $94017-09-01-01$ \\
29 & 475807799.769 & 1.131 & $94017-09-01-01$ \\
30 & 475869743.773 & 0.221 & $94017-09-01-03$ \\
31 & 475875947.685 & 0.055 & $94017-09-01-03$ \\
32 & 476026408.478 & 0.117 & $94017-09-02-01$ \\
33 & 476043593.064 & 0.344 & $94017-09-02-00$ \\
34 & 476063001.703 & 0.066 & $94017-09-02-00$ \\
35 & 476135314.857 & 0.201 & $94017-09-02-02$ \\
36 & 476147642.263 & 0.598 & $94017-09-02-04$ \\
37 & 476147786.107 & 0.316 & $94017-09-02-04$ \\
38 & 476560283.334 & 0.326 & $94017-09-03-00$ \\
39 & 480284479.619 & 0.000 & $94017-09-08-00$ \\
40 & 481034609.390 & 0.000 & $94017-09-09-01$ \\
41 & 481040143.605 & 0.537 & $94017-09-09-00$ \\
42 & 483297895.101 & 0.500 & $94017-09-13-01$ \\
\hline
\end{tabular}


Table 2.2. Observations of Bursts for SGR 1900+14

\begin{tabular}{cccc}
\hline \hline Burst & Start time & $\mathrm{T}_{\text {Bayes }}$ & ObsID \\
ID & in MET & $\mathrm{s}$ & \\
& & & \\
\hline 1 & 139434279.341 & 3.592 & $30197-02-01-00$ \\
2 & 139439198.455 & 0.119 & $30197-02-01-00$ \\
3 & 139607213.183 & 0.094 & $30197-02-01-03$ \\
4 & 146928739.386 & 0.314 & $30197-02-03-00$ \\
5 & 146929244.714 & 0.428 & $30197-02-03-00$ \\
6 & 147003445.879 & 0.000 & $30410-01-02-00$ \\
7 & 147003675.697 & 0.025 & $30410-01-02-00$ \\
8 & 147004718.515 & 0.102 & $30410-01-02-00$ \\
9 & 147007999.167 & 0.209 & $30410-01-02-00$ \\
10 & 147014568.882 & 0.133 & $30410-01-02-00$ \\
11 & 147014638.205 & 0.521 & $30410-01-02-00$ \\
12 & 147015000.218 & 0.930 & $30410-01-02-00$ \\
13 & 147077259.896 & 0.623 & $30410-01-03-00$ \\
14 & 147077547.013 & 3.158 & $30410-01-03-00$ \\
15 & 147079073.416 & 0.963 & $30410-01-03-00$ \\
16 & 147079264.003 & 1.090 & $30410-01-03-00$ \\
17 & 147084263.130 & 0.164 & $30410-01-03-00$ \\
18 & 147085062.919 & 0.203 & $30410-01-03-00$ \\
19 & 147085642.416 & 0.068 & $30410-01-03-00$ \\
20 & 147088754.542 & 0.484 & $30410-01-03-00$ \\
21 & 147090541.925 & 0.123 & $30410-01-03-00$ \\
22 & 147169888.539 & 0.135 & $30410-01-04-00$ \\
23 & 147171715.455 & 0.459 & $30410-01-04-00$ \\
24 & 147172259.263 & 0.121 & $30410-01-04-00$ \\
25 & 147175661.697 & 0.775 & $30410-01-04-00$ \\
26 & 147177608.720 & 0.496 & $30410-01-04-00$ \\
27 & 147180943.384 & 0.150 & $30410-01-04-00$
\end{tabular}


Table 2.2 (cont'd)

\begin{tabular}{cccc}
\hline \hline Burst & Start time & T Bayes & ObsID \\
ID & in MET & $\mathrm{s}$ & \\
& & & \\
\hline 28 & 147181601.363 & 0.441 & $30410-01-04-00$ \\
29 & 147181876.091 & 0.861 & $30410-01-04-00$ \\
30 & 147182415.084 & 0.449 & $30410-01-04-00$ \\
31 & 147182453.917 & 3.305 & $30410-01-04-00$ \\
32 & 147183503.548 & 4.002 & $30410-01-04-00$ \\
33 & 147184062.933 & 0.080 & $30410-01-04-00$ \\
34 & 147246788.400 & 0.137 & $30410-01-05-00$ \\
35 & 147246847.312 & 0.098 & $30410-01-05-00$ \\
36 & 147247016.742 & 0.396 & $30410-01-05-00$ \\
37 & 147247152.951 & 0.666 & $30410-01-05-00$ \\
38 & 147247287.666 & 4.676 & $30410-01-05-00$ \\
39 & 147250097.724 & 0.176 & $30410-01-05-00$ \\
40 & 147250237.996 & 0.916 & $30410-01-05-00$ \\
41 & 147250524.445 & 0.689 & $30410-01-05-00$ \\
42 & 147251054.476 & 0.150 & $30410-01-05-00$ \\
43 & 147251449.642 & 0.100 & $30410-01-05-00$ \\
44 & 147251452.810 & 3.957 & $30410-01-05-00$ \\
45 & 147251709.960 & 0.221 & $30410-01-05-00$ \\
46 & 147252046.871 & 0.166 & $30410-01-05-00$ \\
47 & 147253191.244 & 0.242 & $30410-01-05-00$ \\
48 & 147255733.154 & 0.188 & $30410-01-05-00$ \\
49 & 147257018.589 & 0.426 & $30410-01-05-00$ \\
50 & 147257113.224 & 0.000 & $30410-01-05-00$ \\
51 & 147257600.978 & 1.492 & $30410-01-05-00$ \\
52 & 147259057.048 & 0.391 & $30410-01-05-00$ \\
53 & 147261871.769 & 0.182 & $30410-01-05-00$ \\
54 & 147262125.423 & 0.293 & $30410-01-05-00$
\end{tabular}


Table 2.2 (cont'd)

\begin{tabular}{cccc}
\hline \hline Burst & Start time & T Bayes & ObsID \\
ID & in MET & $\mathrm{s}$ & \\
& & & \\
\hline 55 & 147262574.896 & 0.137 & $30410-01-05-00$ \\
56 & 147263602.074 & 0.521 & $30410-01-05-00$ \\
57 & 147263606.048 & 0.156 & $30410-01-05-00$ \\
58 & 147263682.164 & 0.170 & $30410-01-05-00$ \\
59 & 147263772.185 & 0.164 & $30410-01-05-00$ \\
60 & 147264161.304 & 0.096 & $30410-01-05-00$ \\
61 & 147264612.044 & 0.371 & $30410-01-05-00$ \\
62 & 147264851.630 & 0.000 & $30410-01-05-00$ \\
63 & 147267277.955 & 0.133 & $30410-01-05-00$ \\
64 & 147267816.859 & 3.588 & $30410-01-05-00$ \\
65 & 147268486.353 & 1.057 & $30410-01-05-00$ \\
66 & 147268793.091 & 2.143 & $30410-01-05-00$ \\
67 & 147268816.710 & 0.094 & $30410-01-05-00$ \\
68 & 147269044.771 & 0.131 & $30410-01-05-00$ \\
69 & 147269319.236 & 4.227 & $30410-01-05-00$ \\
70 & 147269619.628 & 0.104 & $30410-01-05-00$ \\
71 & 147269636.861 & 3.336 & $30410-01-05-00$ \\
72 & 147269679.429 & 0.381 & $30410-01-05-00$ \\
73 & 147269694.296 & 0.768 & $30410-01-05-00$ \\
74 & 147269719.591 & 5.635 & $30410-01-05-00$ \\
75 & 147269931.277 & 0.164 & $30410-01-05-00$ \\
76 & 147270287.837 & 0.350 & $30410-01-05-00$ \\
77 & 147270444.955 & 0.830 & $30410-01-05-00$ \\
78 & 147356866.265 & 0.137 & $30410-01-06-00$ \\
79 & 147414276.355 & 0.795 & $30410-01-07-01 \mathrm{R}$ \\
80 & 147511429.707 & 5.762 & $30410-01-08-04$ \\
81 & 147515660.658 & 0.139 & $30410-01-08-00$
\end{tabular}


Table 2.2 (cont'd)

\begin{tabular}{|c|c|c|c|}
\hline $\begin{array}{c}\text { Burst } \\
\text { ID }\end{array}$ & $\begin{array}{l}\text { Start time } \\
\text { in MET }\end{array}$ & $\begin{array}{c}\mathrm{T}_{\text {Bayes }} \\
\mathrm{s}\end{array}$ & ObsID \\
\hline 82 & 147516084.607 & 0.209 & $30410-01-08-00$ \\
\hline 83 & 147867061.392 & 2.518 & $30410-01-09-00$ \\
\hline 84 & 147875361.084 & 2.307 & $30410-01-09-00$ \\
\hline 85 & 148213447.421 & 0.818 & $30410-01-11-00$ \\
\hline 86 & 148386497.900 & 0.975 & $30410-01-13-00$ \\
\hline 87 & 148387169.615 & 0.070 & $30410-01-13-00$ \\
\hline 88 & 148598029.146 & 0.180 & $30410-01-16-00$ \\
\hline 89 & 148634402.974 & 1.607 & $30410-01-17-00$ \\
\hline 90 & 148641419.662 & 0.623 & $30410-01-17-00$ \\
\hline 91 & 154784283.031 & 0.348 & $30410-01-30-00$ \\
\hline 92 & 154786214.597 & 0.744 & $30410-01-30-00$ \\
\hline 93 & 156835490.988 & 0.146 & $30410-01-33-00$ \\
\hline 94 & 157952514.712 & 0.072 & $40130-02-01-00$ \\
\hline 95 & 230367272.294 & 0.334 & $60122-02-01-00$ \\
\hline 96 & 230368050.621 & 0.180 & $60122-02-01-00$ \\
\hline 97 & 230417186.732 & 0.299 & $60122-02-01-01$ \\
\hline 98 & 230578019.683 & 0.422 & $60122-02-01-03$ \\
\hline 99 & 230674705.826 & 0.566 & $60122-02-01-05$ \\
\hline 100 & 231038200.902 & 0.062 & $60122-02-03-01$ \\
\hline 101 & 236866242.511 & 1.449 & $60121-02-02-10$ \\
\hline 102 & 236870495.064 & 0.564 & $60121-02-02-11$ \\
\hline 103 & 237656402.568 & 0.391 & $60121-02-02-01$ \\
\hline 104 & 237759260.050 & 0.098 & $60121-02-02-16$ \\
\hline 105 & 238165998.214 & 0.047 & $60121-02-02-19$ \\
\hline 106 & 257380975.384 & 0.092 & $60122-02-06-02$ \\
\hline 107 & 278740071.910 & 0.162 & $70136-01-15-00$ \\
\hline 108 & 280822633.640 & 3.625 & $70136-01-24-00$ \\
\hline
\end{tabular}


Table 2.2 (cont'd)

\begin{tabular}{cccc}
\hline \hline $\begin{array}{c}\text { Burst } \\
\text { ID }\end{array}$ & $\begin{array}{c}\text { Start time } \\
\text { in MET }\end{array}$ & $\begin{array}{c}\text { T } \text { Bayes } \\
\mathrm{s}\end{array}$ & ObsID \\
& & & \\
\hline 109 & 386183977.169 & 0.000 & $92017-01-01-00$ \\
110 & 386185902.367 & 0.145 & $92017-01-01-00$ \\
111 & 386191213.009 & 0.414 & $92017-01-01-00$ \\
112 & 386223878.003 & 0.291 & $92017-01-02-00$ \\
113 & 386224098.218 & 0.754 & $92017-01-02-00$ \\
114 & 386224496.048 & 0.242 & $92017-01-02-00$ \\
115 & 386224522.607 & 1.412 & $92017-01-02-00$ \\
116 & 386224790.447 & 0.410 & $92017-01-02-00$ \\
117 & 386224857.576 & 0.188 & $92017-01-02-00$ \\
118 & 386226130.023 & 0.137 & $92017-01-02-00$ \\
119 & 386226851.701 & 0.084 & $92017-01-02-00$ \\
120 & 386229325.337 & 0.494 & $92017-01-02-00$ \\
121 & 386229741.619 & 0.707 & $92017-01-02-00$ \\
122 & 386229747.253 & 0.215 & $92017-01-02-00$ \\
123 & 386236643.214 & 0.650 & $92017-01-02-00$ \\
124 & 386236827.429 & 0.146 & $92017-01-02-00$ \\
125 & 387656164.134 & 0.809 & $92017-01-08-02$ \\
\hline
\end{tabular}


Table 2.3. Observations of Bursts for SGR 1806-20

\begin{tabular}{cccc}
\hline \hline Burst & Start time & $\mathrm{T}_{\text {Bayes }}$ & ObsID \\
ID & in MET & $\mathrm{s}$ & \\
& & & \\
\hline 1 & 168976265.693 & 0.305 & $40130-04-13-00$ \\
2 & 173045819.275 & 0.047 & $40130-04-20-00$ \\
3 & 208723242.666 & 0.166 & $50142-01-33-00$ \\
4 & 212193703.626 & 0.023 & $50142-01-43-00$ \\
5 & 212194516.810 & 3.184 & $50142-01-43-00$ \\
6 & 301268310.459 & 0.219 & $80150-01-03-01$ \\
7 & 319514320.580 & 0.186 & $70136-02-02-00$ \\
8 & 328015852.904 & 1.254 & $70136-02-03-00$ \\
9 & 328025618.775 & 1.404 & $70136-02-03-00$ \\
10 & 328027837.027 & 1.221 & $70136-02-03-00$ \\
11 & 328030962.378 & 0.092 & $70136-02-03-00$ \\
12 & 328275286.781 & 0.500 & $90073-02-04-00 G$ \\
13 & 328275618.076 & 0.174 & $90073-02-04-00 G$ \\
14 & 328276403.849 & 0.354 & $90073-02-04-00 G$ \\
15 & 328293954.007 & 0.082 & $90073-02-04-00 G$ \\
16 & 328305819.427 & 0.750 & $90073-02-04-00$ \\
17 & 328311441.347 & 0.488 & $90073-02-04-00$ \\
18 & 328315446.330 & 0.408 & $90073-02-04-00$ \\
19 & 328548898.525 & 0.154 & $70136-02-04-00$ \\
20 & 328807661.400 & 0.418 & $90074-02-01-00$ \\
21 & 328814012.273 & 0.250 & $90074-02-01-00$ \\
22 & 328815824.498 & 0.600 & $90074-02-01-00$ \\
23 & 328894960.964 & 0.947 & $90074-01-02-00$ \\
24 & 328895021.132 & 0.758 & $90074-01-02-00$ \\
25 & 328895130.828 & 0.205 & $90074-01-02-00$ \\
26 & 328898480.791 & 0.281 & $90074-01-02-00$ \\
27 & 328904953.703 & 0.482 & $90074-01-02-00$
\end{tabular}


Table 2.3 (cont'd)

\begin{tabular}{cccc}
\hline \hline $\begin{array}{c}\text { Burst } \\
\text { ID }\end{array}$ & $\begin{array}{c}\text { Start time } \\
\text { in MET }\end{array}$ & $\begin{array}{c}\text { T } \text { Bayes } \\
\text { s }\end{array}$ & ObsID \\
\hline & & & \\
\hline 28 & 328968241.607 & 1.328 & $90074-02-04-00$ \\
29 & 328972004.453 & 1.105 & $90074-02-04-00$ \\
30 & 328973978.935 & 0.219 & $90074-02-04-00$ \\
31 & 328974246.085 & 8.326 & $90074-02-04-00$ \\
32 & 328977705.650 & 0.266 & $90074-02-04-00$ \\
33 & 328980594.308 & 0.170 & $90074-02-04-00$ \\
34 & 328984223.421 & 6.646 & $90074-02-04-00$ \\
35 & 329057295.703 & 3.123 & $90074-02-05-00$ \\
36 & 329058105.849 & 0.279 & $90074-02-05-00$ \\
37 & 329059011.986 & 0.676 & $90074-02-05-00$ \\
38 & 329063564.351 & 0.479 & $90074-02-05-00$ \\
39 & 329064144.406 & 0.928 & $90074-02-05-00$ \\
40 & 329329718.402 & 0.457 & $70136-02-05-00$ \\
41 & 329330714.896 & 2.039 & $70136-02-05-00$ \\
42 & 329331678.345 & 4.963 & $70136-02-05-00$ \\
43 & 329693669.748 & 1.010 & $70136-02-06-00$ \\
44 & 329857129.533 & 0.113 & $90073-02-05-00$ \\
45 & 329863944.501 & 0.285 & $90073-02-05-00$ \\
46 & 330351951.173 & 0.275 & $90073-02-06-00$ \\
47 & 330356347.757 & 0.412 & $90073-02-06-00$ \\
48 & 330356621.453 & 0.287 & $90073-02-06-00$ \\
49 & 330357232.636 & 0.441 & $90073-02-06-00$ \\
50 & 330358372.335 & 0.613 & $90073-02-06-00$ \\
51 & 330362654.258 & 0.000 & $90073-02-06-00$ \\
52 & 330364298.021 & 0.307 & $90073-02-06-00$ \\
53 & 330366879.962 & 0.053 & $90073-02-06-00$ \\
54 & 330368508.224 & 0.049 & $90073-02-06-00$
\end{tabular}


Table 2.3 (cont'd)

\begin{tabular}{cccc}
\hline \hline Burst & Start time & $\mathrm{T}_{\text {Bayes }}$ & ObsID \\
ID & in MET & $\mathrm{S}$ & \\
& & & \\
\hline 55 & 330369519.417 & 0.168 & $90073-02-06-00$ \\
56 & 330531574.691 & 1.490 & $90073-02-06-01$ \\
57 & 330534090.771 & 0.166 & $90073-02-06-01$ \\
58 & 330534227.064 & 0.408 & $90073-02-06-01$ \\
59 & 330800352.136 & 1.062 & $90073-02-07-00$ \\
60 & 331140605.685 & 0.658 & $90073-01-07-01$ \\
61 & 331140923.084 & 0.510 & $90073-01-07-01$ \\
62 & 331145181.308 & 0.119 & $90073-01-07-01$ \\
63 & 331145259.667 & 0.584 & $90073-01-07-01$ \\
64 & 331145597.164 & 0.102 & $90073-01-07-01$ \\
65 & 331389118.271 & 2.117 & $90073-02-08-00$ \\
66 & 331909545.144 & 0.346 & $90073-02-08-02$ \\
67 & 331917786.486 & 0.307 & $90073-02-08-02$ \\
68 & 333015043.376 & 0.432 & $90073-02-10-00$ \\
69 & 333015699.974 & 0.430 & $90073-02-10-00$ \\
70 & 333022654.927 & 3.277 & $90073-02-10-00$ \\
71 & 333446698.396 & 0.221 & $90073-02-11-00$ \\
72 & 333532468.707 & 0.191 & $90073-02-11-01$ \\
73 & 334824007.554 & 0.066 & $80149-02-11-01$ \\
74 & 335000025.187 & 6.404 & $80149-02-12-000$ \\
75 & 335010892.757 & 0.398 & $80149-02-12-000$ \\
76 & 335013738.318 & 0.271 & $80149-02-12-000$ \\
77 & 335019339.707 & 0.125 & $80149-02-12-000$ \\
78 & 335022521.818 & 0.215 & $80149-02-12-000$ \\
79 & 335364358.746 & 0.547 & $80149-02-12-01$ \\
80 & 335776010.851 & 0.668 & $80149-02-13-00$ \\
81 & 335778407.001 & 0.430 & $80149-02-13-00$
\end{tabular}


Table 2.3 (cont'd)

\begin{tabular}{|c|c|c|c|}
\hline $\begin{array}{c}\text { Burst } \\
\text { ID }\end{array}$ & $\begin{array}{l}\text { Start time } \\
\text { in MET }\end{array}$ & $\begin{array}{c}\mathrm{T}_{\text {Bayes }} \\
\mathrm{s}\end{array}$ & ObsID \\
\hline 82 & 336043755.548 & 1.785 & 80149-02-13-01 \\
\hline 83 & 336044128.517 & 0.441 & 80149-02-13-01 \\
\hline 84 & 336276921.783 & 0.227 & $70136-02-07-00$ \\
\hline 85 & 336277747.943 & 0.047 & 70136-02-07-00 \\
\hline 86 & 336294246.630 & 0.844 & 70136-02-07-02 \\
\hline 87 & 336696548.716 & 0.256 & $70136-02-08-00$ \\
\hline 88 & 336706832.871 & 0.209 & 70136-02-08-00 \\
\hline 89 & 336985822.966 & 0.533 & $90074-02-06-00$ \\
\hline 90 & 336996597.666 & 1.166 & $90074-02-06-00$ \\
\hline 91 & 336996828.585 & 0.098 & $90074-02-06-00$ \\
\hline 92 & 337103764.689 & 0.625 & $90074-02-08-00$ \\
\hline 93 & 337111577.630 & 1.035 & 90074-02-08-01 \\
\hline 94 & 337116929.220 & 3.736 & 90074-02-09-00 \\
\hline 95 & 337127242.667 & 0.242 & 90074-02-09-00 \\
\hline 96 & 337127437.849 & 0.107 & 90074-02-09-00 \\
\hline 97 & 337128192.460 & 0.092 & $90074-02-09-00$ \\
\hline 98 & 337194856.871 & 0.168 & $90074-02-10-01$ \\
\hline 99 & 337195562.302 & 0.547 & 90074-02-10-01 \\
\hline 100 & 337200104.705 & 1.184 & $90074-02-10-00$ \\
\hline 101 & 337200331.162 & 0.426 & $90074-02-10-00$ \\
\hline 102 & 337200882.531 & 0.479 & $90074-02-10-00$ \\
\hline 103 & 337201096.220 & 1.293 & $90074-02-10-00$ \\
\hline 104 & 337207442.175 & 0.234 & $90074-02-10-00$ \\
\hline 105 & 337511420.304 & 0.391 & 70136-02-09-00 \\
\hline 106 & 337513415.291 & 0.256 & 70136-02-09-00 \\
\hline 107 & 337873741.544 & 1.607 & $70136-02-10-00$ \\
\hline 108 & 337887686.337 & 0.203 & 70136-02-10-02 \\
\hline
\end{tabular}


Table 2.3 (cont'd)

\begin{tabular}{cccc}
\hline \hline $\begin{array}{c}\text { Burst } \\
\text { ID }\end{array}$ & $\begin{array}{c}\text { Start time } \\
\text { in MET }\end{array}$ & $\begin{array}{c}\text { T } \text { Bayes } \\
\mathrm{S}\end{array}$ & ObsID \\
& & & \\
\hline 109 & 338390837.962 & 0.125 & $80149-02-14-00$ \\
110 & 338657331.916 & 0.240 & $80149-02-15-00$ \\
111 & 339665575.576 & 5.596 & $80149-02-16-00$ \\
112 & 339666254.238 & 0.482 & $80149-02-16-00$ \\
113 & 339666508.816 & 0.736 & $80149-02-16-00$ \\
114 & 339667274.210 & 0.098 & $80149-02-16-00$ \\
115 & 339667301.746 & 0.201 & $80149-02-16-00$ \\
116 & 339671612.923 & 0.068 & $80149-02-16-00$ \\
117 & 339671639.703 & 0.336 & $80149-02-16-00$ \\
118 & 339672403.667 & 2.602 & $80149-02-16-00$ \\
119 & 339672447.150 & 0.172 & $80149-02-16-00$ \\
120 & 339672598.585 & 0.236 & $80149-02-16-00$ \\
121 & 339672754.494 & 0.312 & $80149-02-16-00$ \\
122 & 339672894.449 & 5.893 & $80149-02-16-00$ \\
123 & 339672982.574 & 0.297 & $80149-02-16-00$ \\
124 & 339673078.859 & 0.143 & $80149-02-16-00$ \\
125 & 339673458.498 & 2.191 & $80149-02-16-00$ \\
126 & 339673484.687 & 0.137 & $80149-02-16-00$ \\
127 & 339673896.446 & 0.000 & $80149-02-16-00$ \\
128 & 339746730.484 & 0.090 & $80149-02-16-01$ \\
129 & 339916908.501 & 0.488 & $70136-02-11-00$ \\
130 & 340267873.919 & 0.213 & $90074-02-11-01$ \\
131 & 340351599.419 & 0.236 & $90074-02-11-02$ \\
132 & 340608599.818 & 0.248 & $90074-02-12-00$ \\
133 & 340612607.759 & 0.000 & $90074-02-12-00$ \\
134 & 340612616.884 & 0.000 & $90074-02-12-00$ \\
135 & 340612697.751 & 0.953 & $90074-02-12-00$
\end{tabular}


Table 2.3 (cont'd)

\begin{tabular}{cccc}
\hline \hline $\begin{array}{c}\text { Burst } \\
\text { ID }\end{array}$ & $\begin{array}{c}\text { Start time } \\
\text { in MET }\end{array}$ & $\begin{array}{c}\text { T } \text { Bayes } \\
\text { S }\end{array}$ & ObsID \\
& & & \\
\hline 136 & 340612733.290 & 0.000 & $90074-02-12-00$ \\
137 & 340612966.781 & 1.697 & $90074-02-12-00$ \\
138 & 340613037.852 & 0.000 & $90074-02-12-00$ \\
139 & 340613175.821 & 0.000 & $90074-02-12-00$ \\
140 & 340613750.412 & 0.600 & $90074-02-12-00$ \\
141 & 340999953.724 & 0.225 & $90074-02-12-01$ \\
142 & 341003003.517 & 0.611 & $90074-02-12-02 \mathrm{G}$ \\
143 & 341004127.492 & 0.223 & $90074-02-12-02 \mathrm{G}$ \\
144 & 341259209.189 & 1.719 & $90074-02-13-00$ \\
145 & 341824583.607 & 0.236 & $90074-02-14-00$ \\
146 & 342165817.738 & 0.109 & $90074-02-14-01$ \\
147 & 342171181.298 & 0.162 & $90074-02-14-01$ \\
148 & 342755100.162 & 0.152 & $90074-02-15-01$ \\
149 & 342756231.416 & 1.846 & $90074-02-15-01$ \\
150 & 342760693.384 & 0.613 & $90074-02-15-01$ \\
151 & 343723618.242 & 0.219 & $70136-02-13-01$ \\
152 & 349039848.671 & 0.150 & $91065-01-01-000$ \\
153 & 349063562.855 & 0.660 & $91065-01-01-00$ \\
154 & 349067593.917 & 0.518 & $91065-01-01-00$ \\
155 & 349221450.058 & 0.264 & $91065-01-01-02$ \\
156 & 349222664.119 & 0.305 & $91065-01-01-02$ \\
157 & 349225923.259 & 0.188 & $91065-01-01-02$ \\
158 & 349287908.390 & 0.295 & $91065-01-01-03$ \\
159 & 349288451.349 & 0.205 & $91065-01-01-03$ \\
160 & 362758378.281 & 0.172 & $91062-02-09-00$ \\
161 & 363089104.177 & 0.096 & $91062-02-11-00$ \\
162 & 363190455.785 & 0.125 & $91062-02-12-00$
\end{tabular}


Table 2.3 (cont'd)

\begin{tabular}{|c|c|c|c|}
\hline $\begin{array}{c}\text { Burst } \\
\text { ID }\end{array}$ & $\begin{array}{l}\text { Start time } \\
\text { in MET }\end{array}$ & $\begin{array}{c}\mathrm{T}_{\text {Bayes }} \\
\mathrm{s}\end{array}$ & ObsID \\
\hline 163 & 363454444.033 & 0.072 & $91062-02-13-00$ \\
\hline 164 & 366541462.853 & 0.260 & $91065-01-05-00$ \\
\hline 165 & 366542553.179 & 0.357 & $91065-01-05-00$ \\
\hline 166 & 371055794.474 & 0.836 & $91065-01-06-02$ \\
\hline 167 & 389717164.886 & 1.986 & $92015-02-02-00$ \\
\hline 168 & 390182002.966 & 0.119 & $92015-02-05-00$ \\
\hline 169 & 396371066.191 & 4.455 & $92017-02-01-00$ \\
\hline 170 & 397633576.568 & 0.119 & $92017-02-02-00$ \\
\hline 171 & 398067978.826 & 0.189 & $92017-02-03-00$ \\
\hline 172 & 398069005.332 & 0.184 & $92017-02-03-00$ \\
\hline 173 & 398069382.578 & 0.182 & $92017-02-03-00$ \\
\hline 174 & 399660227.943 & 0.111 & $92017-02-04-00$ \\
\hline 175 & 399833977.718 & 0.229 & $92017-02-05-00$ \\
\hline 176 & 402017394.441 & 0.293 & $92015-02-08-00$ \\
\hline 177 & 402019525.789 & 0.658 & $92015-02-08-00$ \\
\hline 178 & 402028823.068 & 0.000 & $92015-02-08-00$ \\
\hline 179 & 402029256.359 & 0.188 & $92015-02-08-00$ \\
\hline 180 & 402030789.677 & 0.033 & $92015-02-08-00$ \\
\hline 181 & 402614211.646 & 0.316 & $92015-02-09-00$ \\
\hline 182 & 402614229.544 & 0.152 & $92015-02-09-00$ \\
\hline 183 & 402996582.300 & 0.088 & $92015-02-12-00$ \\
\hline 184 & 403004861.091 & 0.967 & $92015-02-12-00$ \\
\hline 185 & 403802005.443 & 0.457 & $92015-02-14-00$ \\
\hline 186 & 415917635.253 & 1.736 & $92015-02-16-00$ \\
\hline 187 & 416360422.888 & 3.729 & $92015-02-18-00$ \\
\hline 188 & 417225091.140 & 7.152 & $92015-02-21-00$ \\
\hline 189 & 418673271.802 & 0.594 & $92017-02-07-00$ \\
\hline
\end{tabular}


Table 2.3 (cont'd)

\begin{tabular}{cccc}
\hline \hline $\begin{array}{c}\text { Burst } \\
\text { ID }\end{array}$ & $\begin{array}{c}\text { Start time } \\
\text { in MET }\end{array}$ & $\begin{array}{c}\mathrm{T}_{\text {Bayes }} \\
\mathrm{S}\end{array}$ & ObsID \\
& & & \\
\hline 190 & 429186756.941 & 0.053 & $93016-01-04-00$ \\
191 & 432936365.980 & 0.045 & $93048-01-01-00$ \\
192 & 433360490.830 & 0.094 & $93048-01-03-00$ \\
193 & 433372006.550 & 0.336 & $93048-01-03-00$ \\
194 & 89829138.386 & 3.412 & $20165-01-01-000$ \\
195 & 89830941.550 & 0.217 & $20165-01-01-000$ \\
196 & 89831926.810 & 0.256 & $20165-01-01-000$ \\
197 & 89832172.201 & 0.344 & $20165-01-01-000$ \\
198 & 89832442.017 & 0.730 & $20165-01-01-000$ \\
199 & 89832668.882 & 0.357 & $20165-01-01-000$ \\
200 & 89836026.320 & 0.555 & $20165-01-01-000$ \\
201 & 89872404.599 & 0.221 & $20165-01-01-002$ \\
202 & 90835073.441 & 0.215 & $10223-01-03-000$ \\
203 & 90919656.808 & 0.846 & $10223-01-03-01$ \\
204 & 90921319.876 & 0.975 & $10223-01-03-01$ \\
205 & 90921613.044 & 5.109 & $10223-01-03-01$ \\
206 & 90925020.707 & 0.414 & $10223-01-03-01$ \\
207 & 90926041.330 & 0.488 & $10223-01-03-01$ \\
208 & 90931800.996 & 1.441 & $10223-01-03-01$ \\
209 & 90932073.783 & 0.209 & $10223-01-03-01$ \\
210 & 90932840.658 & 9.176 & $10223-01-03-01$ \\
211 & 90933295.505 & 8.398 & $10223-01-03-01$ \\
212 & 90935941.416 & 0.537 & $10223-01-03-01$ \\
213 & 90936244.113 & 0.846 & $10223-01-03-01$ \\
214 & 90936381.205 & 0.330 & $10223-01-03-01$ \\
215 & 90937280.673 & 0.488 & $10223-01-03-01$ \\
216 & 90937870.931 & 5.125 & $10223-01-03-01$
\end{tabular}


Table 2.3 (cont'd)

\begin{tabular}{cccc}
\hline \hline $\begin{array}{c}\text { Burst } \\
\text { ID }\end{array}$ & $\begin{array}{c}\text { Start time } \\
\text { in MET }\end{array}$ & $\begin{array}{c}\text { T Bayes } \\
\mathrm{s}\end{array}$ & ObsID \\
& & & \\
\hline 217 & 90938174.242 & 4.238 & $10223-01-03-01$ \\
218 & 90938565.296 & 1.873 & $10223-01-03-01$ \\
219 & 90938969.390 & 0.316 & $10223-01-03-01$ \\
220 & 90939118.126 & 0.506 & $10223-01-03-01$ \\
221 & 90941708.416 & 3.391 & $10223-01-03-01$ \\
\hline
\end{tabular}




\section{Chapter 3}

\section{Spectral Analysis and Results}

\subsection{Continuum Models}

In our broadband spectral analysis, we used four continuum models. Three of which have been commonly used in describing short magnetar bursts in previous studies: The sum of two blackbody functions $(\mathrm{BB}+\mathrm{BB})$, sum of blackbody and power law models $(\mathrm{BB}+\mathrm{PO})$, Comptonized model (COMPT). Additionally, we also employed the sum of two modified blackbody functions (LB + LB) as set forth by Lyubarsky 2002. Note that the COMPT model is simply a power law with a high energy exponential cutoff expressed as:

$$
f=A E^{-\alpha} \exp \left(-E / E_{c u t}\right)
$$

where $\mathrm{f}$ is the photon flux and $\mathrm{A}$ is the amplitude in photons $/ \mathrm{cm}^{2} / \mathrm{s} / \mathrm{keV}$ at $1 \mathrm{keV}, E_{\text {cut }}$ is the cutoff energy (in $\mathrm{keV}$ ) and $\alpha$ is the photon index.

The LB function is a modified version of the blackbody function where the spectrum is flattened at low energies. In terms of the photon flux, the function is expressed as:

$$
f=0.47 \varepsilon^{2}\left[\exp \left(\frac{\varepsilon^{2}}{T_{b} \sqrt{\varepsilon^{2}+\left(3 \pi^{2} / 5\right) T_{b}^{2}}}\right)-1\right]^{-1}
$$

where, $T_{b}$ is the bolometric temperature in $\mathrm{keV}$ and $\varepsilon$ is the photon energy (see Section 4.2 for a brief theoretical description of the LB model and Lyubarsky 2002 for the detailed theoretical model definition). To display intrinsic differences of these continuum models, we present in Figure 3.1 the model curves generated with the fitted parameters for the event with Burst ID: 79 observed from SGR 1806-20. In Figure 3.2, we introduce the broadband spectrum of the same burst along with the fit residuals of all these four continuum models as an example. 


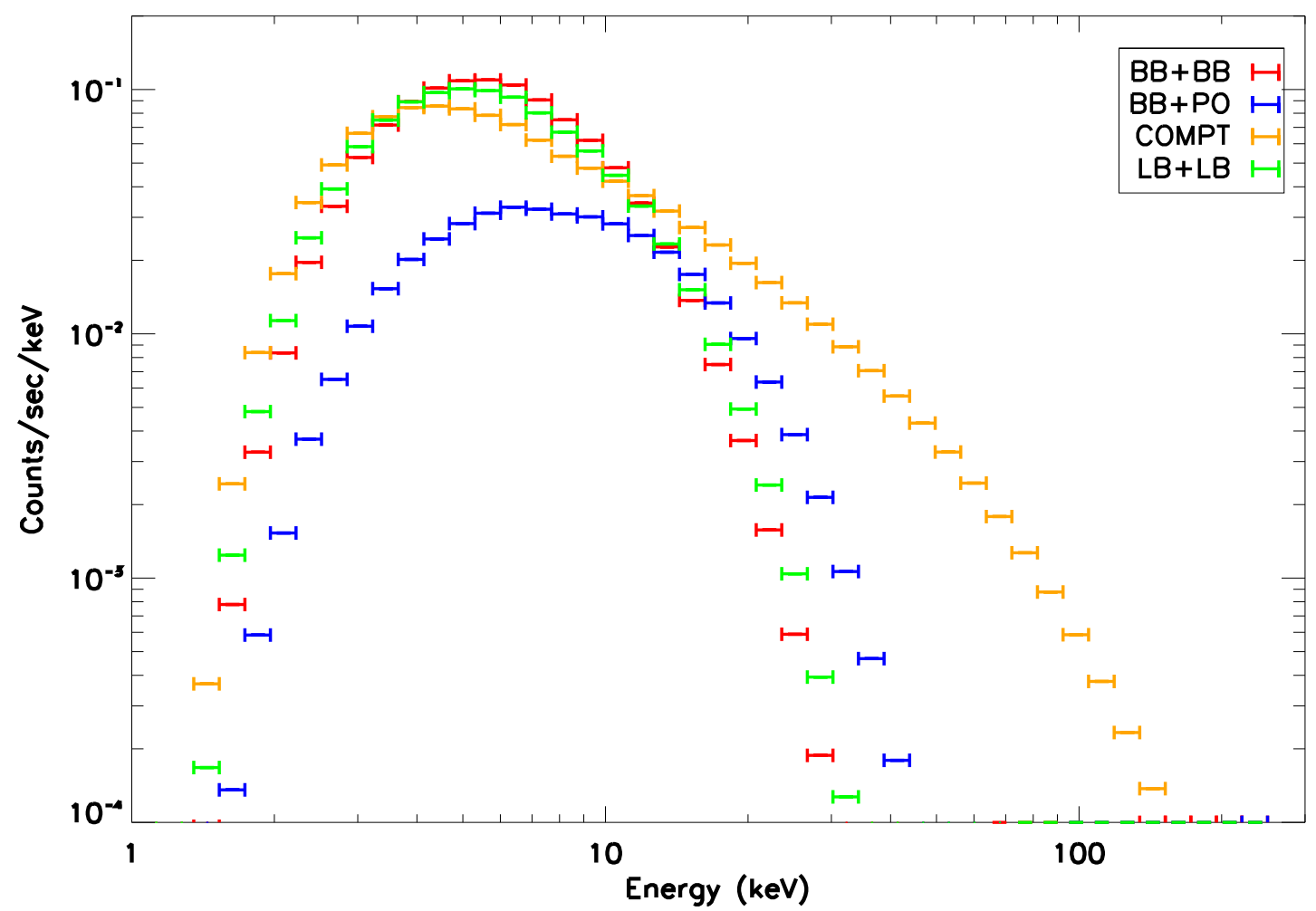

Figure 3.1 Model curves for SGR1806-20 Burst Start Time (MET): 335364358.746

To address any cross-calibration incompatibility between PCA and HEXTE detector responses, we performed joint spectral analysis with a small sample (11) of bursts. In this task, we introduced a multiplicative constant for HEXTE parameters to account for such incompatibility. We repeated the same analysis with the same burst sample without this scaling term. We found that the spectral analysis results with and without the constant term are in agreement with each other within errors. Therefore, we proceeded our investigations without including the constant scale factor. 


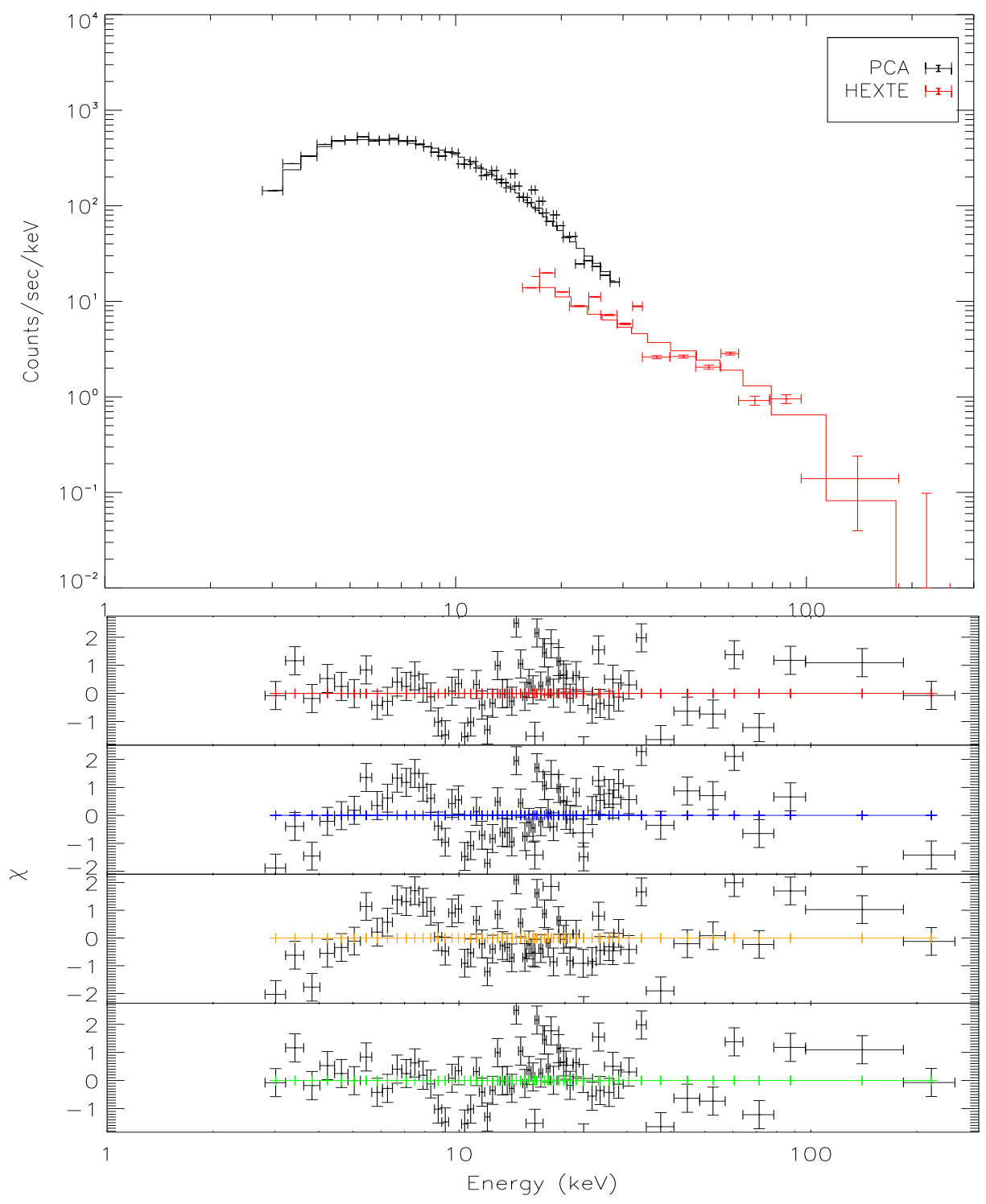

Figure 3.2 Top panel: Fitted models for SGR1806-20 Burst Start Time (MET): 335364358.746. Lower group of panels: Fit residuals for the same event. The models for fit residuals are $\mathrm{BB}+\mathrm{BB}$, $\mathrm{BB}+\mathrm{PO}, \mathrm{COMPT}, \mathrm{LB}+\mathrm{LB}$ respectively from top to bottom. 
Table 3.1. Percentage of acceptable spectral fits based on $\chi^{2}$ probability for the given DOF

\begin{tabular}{cccc}
\hline \hline Model & SGR J1550-5418 & SGR 1900+14 & 1806-20 \\
& & & \\
\hline BB+PO & $73.8 \%$ & $72.8 \%$ & $66.0 \%$ \\
BB+BB & $61.9 \%$ & $69.6 \%$ & $69.2 \%$ \\
LB+LB & $71.4 \%$ & $83.2 \%$ & $78.7 \%$ \\
COMPT & $71.4 \%$ & $77.6 \%$ & $67.9 \%$ \\
\hline
\end{tabular}

\subsection{Results}

In this section, we report resulting spectral parameters of all four models, along with associated fit statistics for each burst of the three magnetars. Note that we report errors calculated at $1 \sigma$. We calculated interstellar neutral hydrogen absorption corrected fluxes for PCA and HEXTE in the energy intervals in which the spectral fits were performed and using spectral parameters of the COMPT model fit. Detailed statistical investigations were possible for large burst samples (SGR 1900+14 and SGR 1806-20). However, the number of events sample suitable for spectral analysis was not sufficient to provide reliable distributions for SGR J1550-5418 burst spectral parameters. Therefore, we present parameter, flux, fluence and energy distributions for SGR 1900+14 and SGR 1806-20 and report statistical results of parameters and flux for SGR J1550-5418 only. We have also presented our results as a database at http://magnetars . sabanciuniv. edu. It is also important to note that our burst samples involve partially saturated bursts. In each of those cases, the reported burst flux should be taken as a lower bound.

In general, we find that all of the four composite models can successfully describe most of the bursts from for all three sources based on the resulting $\chi^{2}$ statistics. This can be seen in Table 3.1, in which we present the percentage of spectra that resulted in statistically acceptable fits for each model. Here, we define the fits to be "acceptable" when the probability of obtaining $\chi^{2}$ greater than the resulting $\chi^{2}$ value based on the $\chi^{2}$ distribution for the corresponding degrees of freedom (DOF), is greater than 0.2 . This means that the fits that do not match this criteria have unacceptably large $\chi^{2}$ values with a low probability of occuring by chance. We see that all of these models can adequately represent the burst spectra at similar levels. 


\subsubsection{Descriptive Statistics of Spectral Parameters and Flux}

\subsubsection{SGR J1550-5418}

The COMPT and BB+BB models are the most commonly used models in spectral analysis due to their high performance of describing magnetar bursts. In our analysis for SGR J1550-5418, 31 out of 42 bursts had the lowest reduced $\chi^{2}$ values when fitted with the COMPT model, while only one burst had the lowest reduced $\chi^{2}$ value when fitted with the $\mathrm{BB}+\mathrm{BB}$ model.

For the COMPT model, the photon index range from -0.28 to 1.77 with a mean of 1.21 while the exponential cutoff energy range from $4.30 \mathrm{keV}$ to $118.26 \mathrm{keV}$, with an average of $54.46 \mathrm{keV}$. The combined unabsorbed flux (in the $2-250 \mathrm{keV}$ band) varies from $3.72 \times 10^{-9}$ to $2.62 \times 10^{-8}$ erg $\mathrm{cm}^{-2} \mathrm{~s}^{-1}$.

For the $\mathrm{BB}+\mathrm{BB}$ model, the temperature of the cooler component (in $\mathrm{keV}$ ) range from 1.02 to 2.6 with a mean of 1.76, and from 5.67 to 29.24 with a mean of 13.71 for the hot blackbody component. Note that the parameter ranges and averages presented here are excluding fits where either one of the upper or lower bound errors are not available. For more detailed results (i.e. spectral parameters and fit statistics for each burst on all models), see Section 3.3.2.

\subsubsection{SGR 1900+14}

Similar to the SGR J1550-5418 case, we find that a great majority of burst spectra are better described with the COMPT model (98 out of 125 bursts) while the BB+BB model had the lead in only six bursts.

Since SGR $1900+14$ has a larger sample size, we were able to generate spectral parameter distributions. To do so, we selected the events that resulted in errors less than $50 \%$ of the given parameter (both lower and upper bound). We then modeled the distributions with a Gaussian to determine the mean value.

We obtain that distribution of photon indices peak at $0.86 \pm 0.02$ with $\sigma=0.25 \pm 0.02$ (see the top panel of Figure 3.3). The distribution of exponential cutoff energy (Figure 3.3, top panel) yields a mean value of $14.38 \pm 1.0 \mathrm{keV}$ with a width of $\sigma=7.96 \pm 1.1 \mathrm{keV}$. For the BB+BB model, the mean temperature of the cooler blackbody is $1.76 \pm 0.02 \mathrm{keV}(\sigma=0.3 \pm 0.02 \mathrm{keV})$, and the mean temperature of the hotter blackbody is $6.2 \pm 0.2 \mathrm{keV}(\sigma=4.3 \pm 0.2 \mathrm{keV}$ ) (See Figure 3.3, lower panels).

We also computed the $2-250 \mathrm{keV}$ flux for the sample of 125 bursts, and found that they are between $4.02 \times 10^{-9}$ and $6.9 \times 10^{-8} \mathrm{erg} \mathrm{cm}^{-2} \mathrm{~s}^{-1}$ (see Figure 3.4 for individual PCA and HEXTE flux distributions for $\mathrm{BB}+\mathrm{BB}$ (lower panels) and COMPT (upper panels) models). We have also generated fluence (flux $\times$ exposure time) and total energy distributions for COMPT and BB+BB models based on the flux distributions. We report the fitted fluence distributions in Figure 3.5 and 

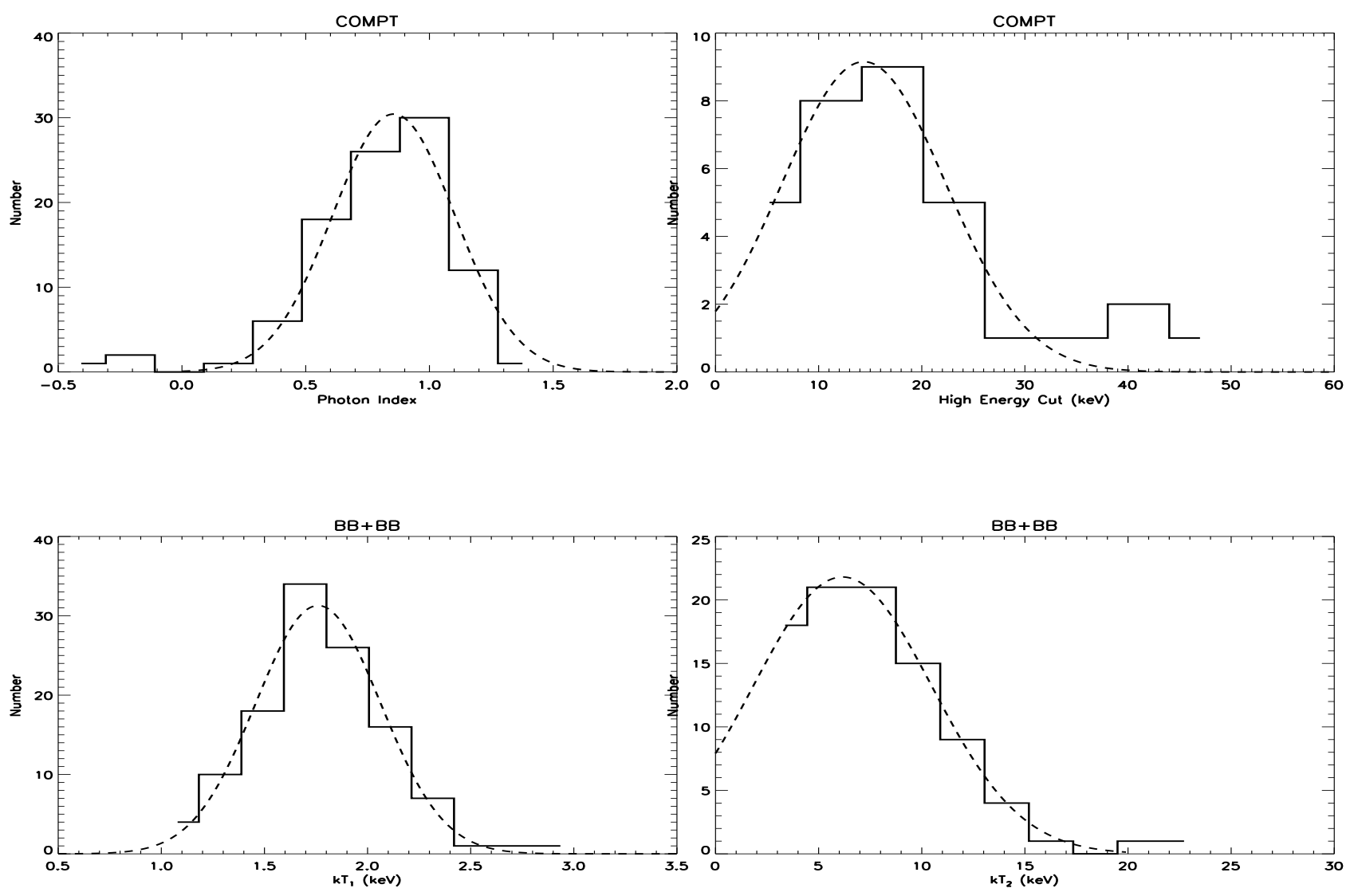

Figure 3.3 SGR 1900+14 Fitted parameter distributions. The top two figures are the distributions of the photon index (left) and high energy cut (right) with a Gaussian fit (dashed lines) for the COMPT model. The two figures on the bottom are the distributions of the temperatures of the cold (left) and hot (right) additive blackbody components for the BB + BB model.

the log-log distributions on total energies emitted from the source in Figure 3.6. We find that the majority of SGR $1900+14$ bursts have fluences $\sim 10^{-8} \mathrm{erg}^{-2}$ with only a few exceptions. 

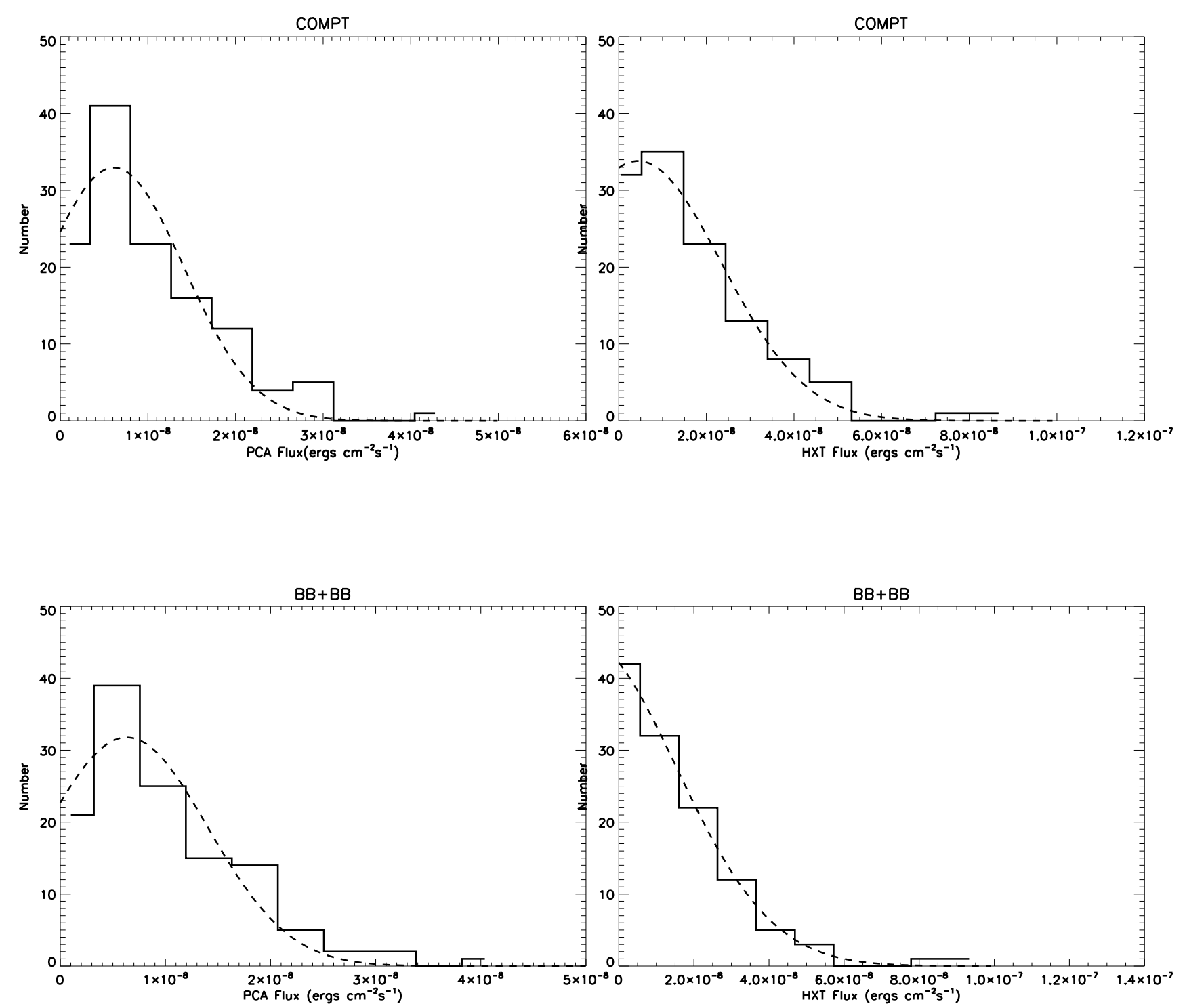

Figure 3.4 SGR 1900+14 Fitted flux distributions for PCA (right) and HEXTE fits (left) for the COMPT (top) and BB+BB (bottom) models. Gaussian fits are shown with dashed lines. 

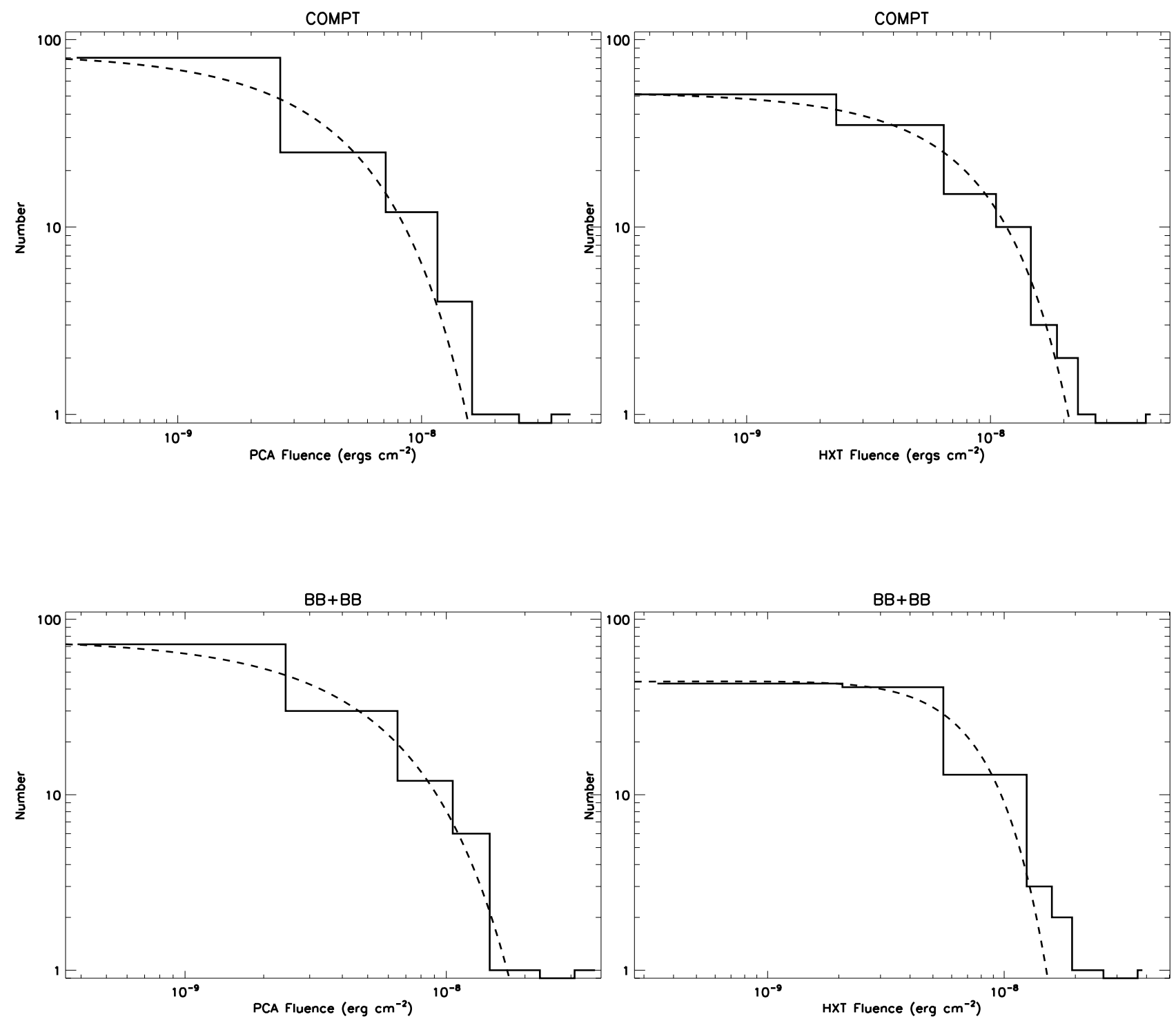

Figure 3.5 SGR 1900+14 Fitted fluence distributions for PCA (right) and HEXTE fits (left) for the COMPT (top) and BB+BB (bottom) models. Gaussian fits are shown with dashed lines. 

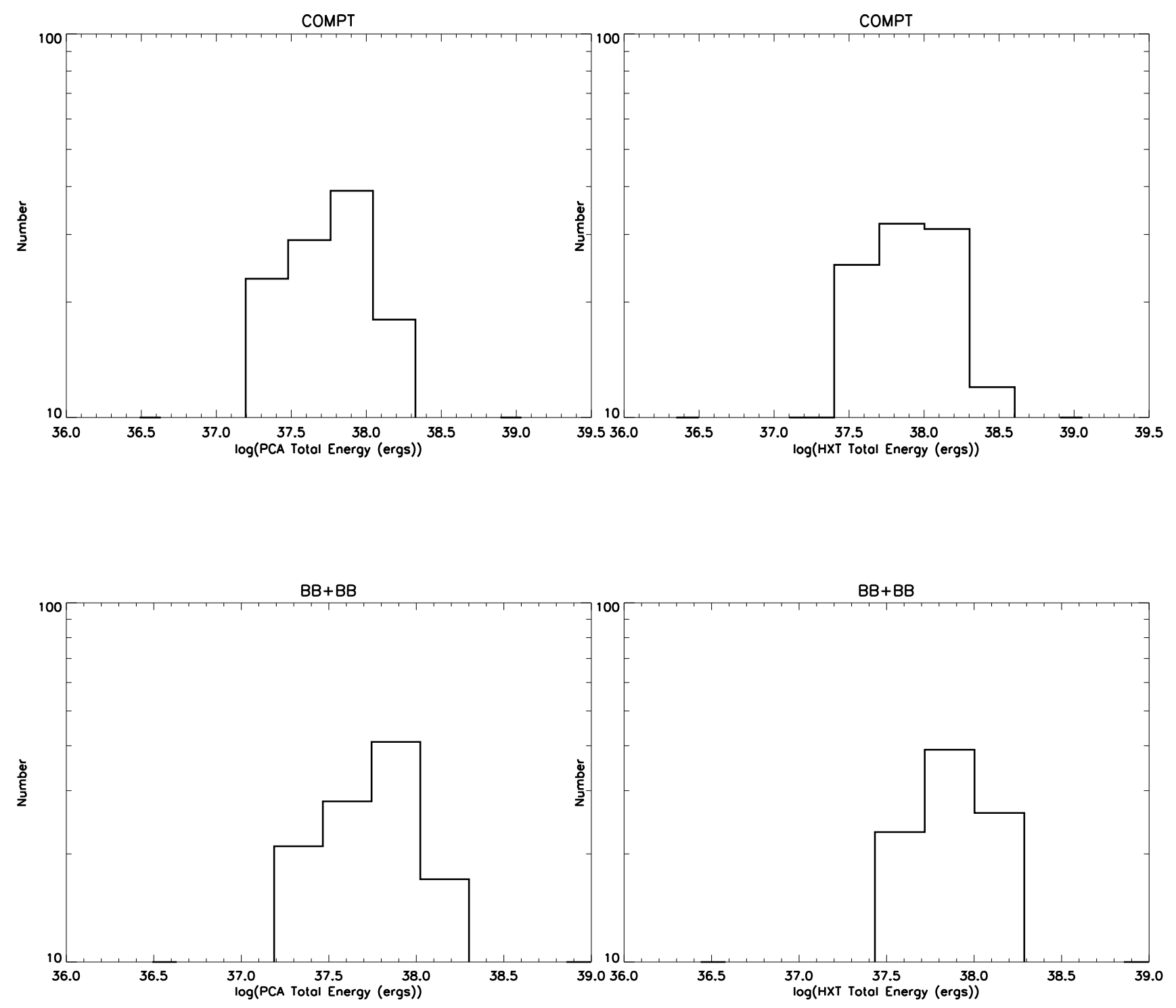

Figure 3.6 SGR 1900+14 Log-log plots of total energy for PCA (right) and HEXTE fits (left) for the COMPT (top) and BB+BB (bottom) models. 


\subsubsection{SGR 1806-20}

The overall trend of fit statistics for SGR 1806-20 resembles that of the other two sources with COMPT model best describing the majority of (129 out of 221) bursts, followed by the BB+BB model leading in 21 bursts. On average, spectral model parameters of SGR 1806-20 bursts span narrower intervals compared to those of SGR 1900+14 bursts.

We generated spectral parameter distributions for SGR 1806-20 with the same procedure as SGR 1900+14. For the COMPT model, we find a photon index distribution mean of $0.62 \pm 0.005$ $(\sigma=0.22 \pm 0.005)$. The exponential cutoff energy distribution peaks at $21.1 \pm 1.3 \mathrm{keV}$ with $\sigma=$ $15.58 \pm 1.5 \mathrm{keV}$. (see Figure 3.7, top panels). The BB+BB model yields a mean cooler blackbody temperature of $2.02 \pm 0.02 \mathrm{keV}$ with $\sigma=0.24 \pm 0.02 \mathrm{keV}$. The mean hotter blackbody temperature is $9.6 \pm 0.2 \mathrm{keV}$ with $\sigma=2.7 \pm 0.2 \mathrm{keV}$. (see Figure 3.7, bottom panels). The 2-250 keV flux of SGR 1806-20 bursts is in the range $4.91 \times 10^{-9}-5.46 \times 10^{-8} \mathrm{erg} \mathrm{cm}^{-2} \mathrm{~s}^{-1}$.

On average, the combined unabsorbed 2-250 keV flux of SGR 1806-20 bursts are higher than SGR J1550-5418 and similar to SGR 1900+14 with a range of $4.91 \times 10^{-9}-5.46 \times 10^{-8} \mathrm{erg} \mathrm{cm}^{-2}$ $\mathrm{s}^{-1}$. Due to the longer average exposure time, burst fluences of SGR 1806-20 events tend to be higher than both SGR1900+14 and SGR J1550-5418.

In figure 3.7 are the joint fit distributions of parameter values for SGR 1806-20. For the resulting flux, fluence and energy distributions with the same models see Figures 3.8, 3.9 and 3.10 respectively. 

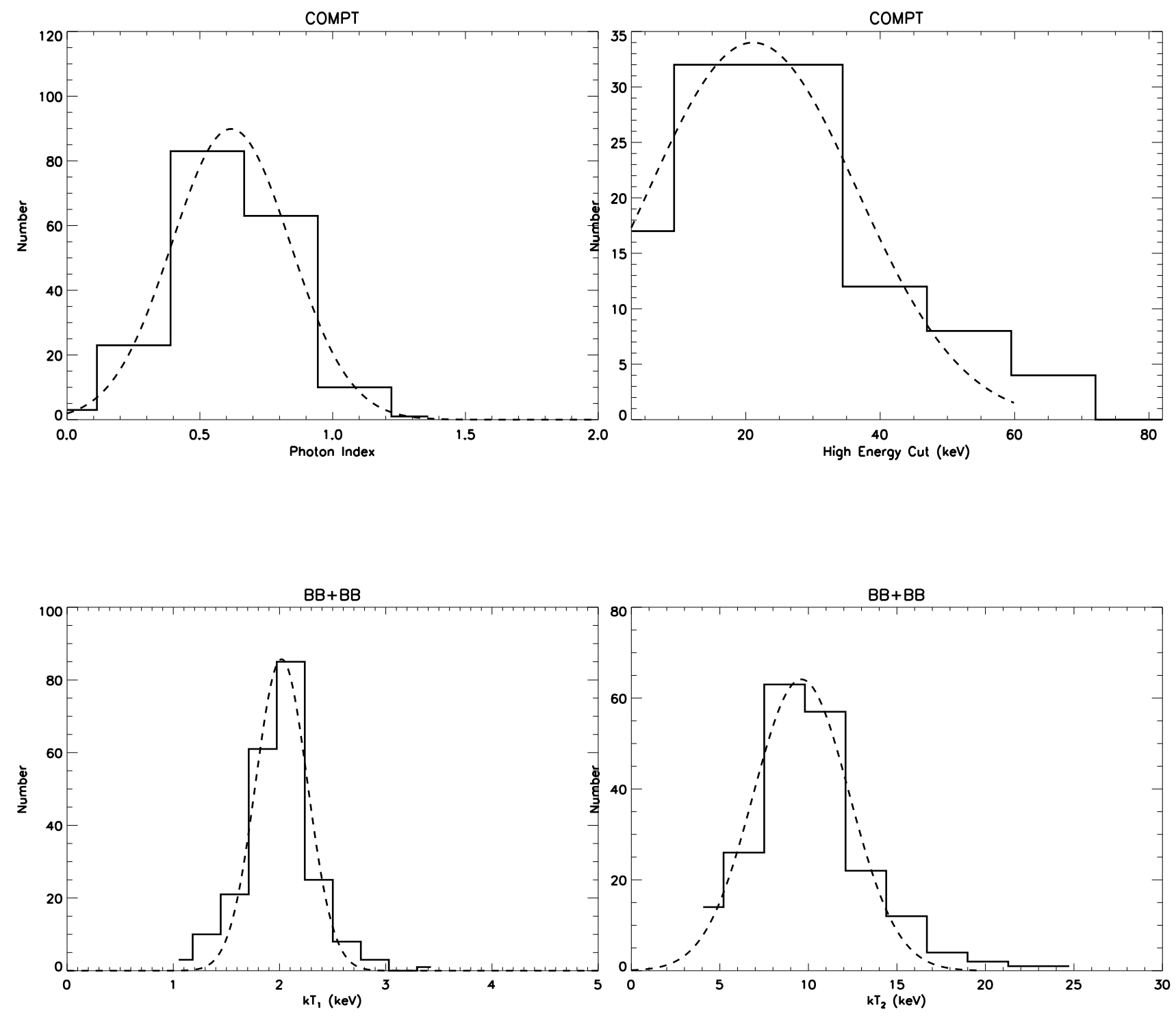

Figure 3.7 SGR 1806-20 Fitted parameter distributions. The top two figures are the distributions of the photon index (left) and high energy cut (right) with a Gaussian fit (dashed lines) for the COMPT model. The two figures on the bottom are the distributions of the temperatures of the cold (left) and hot (right) additive blackbody components for the BB + BB model. 

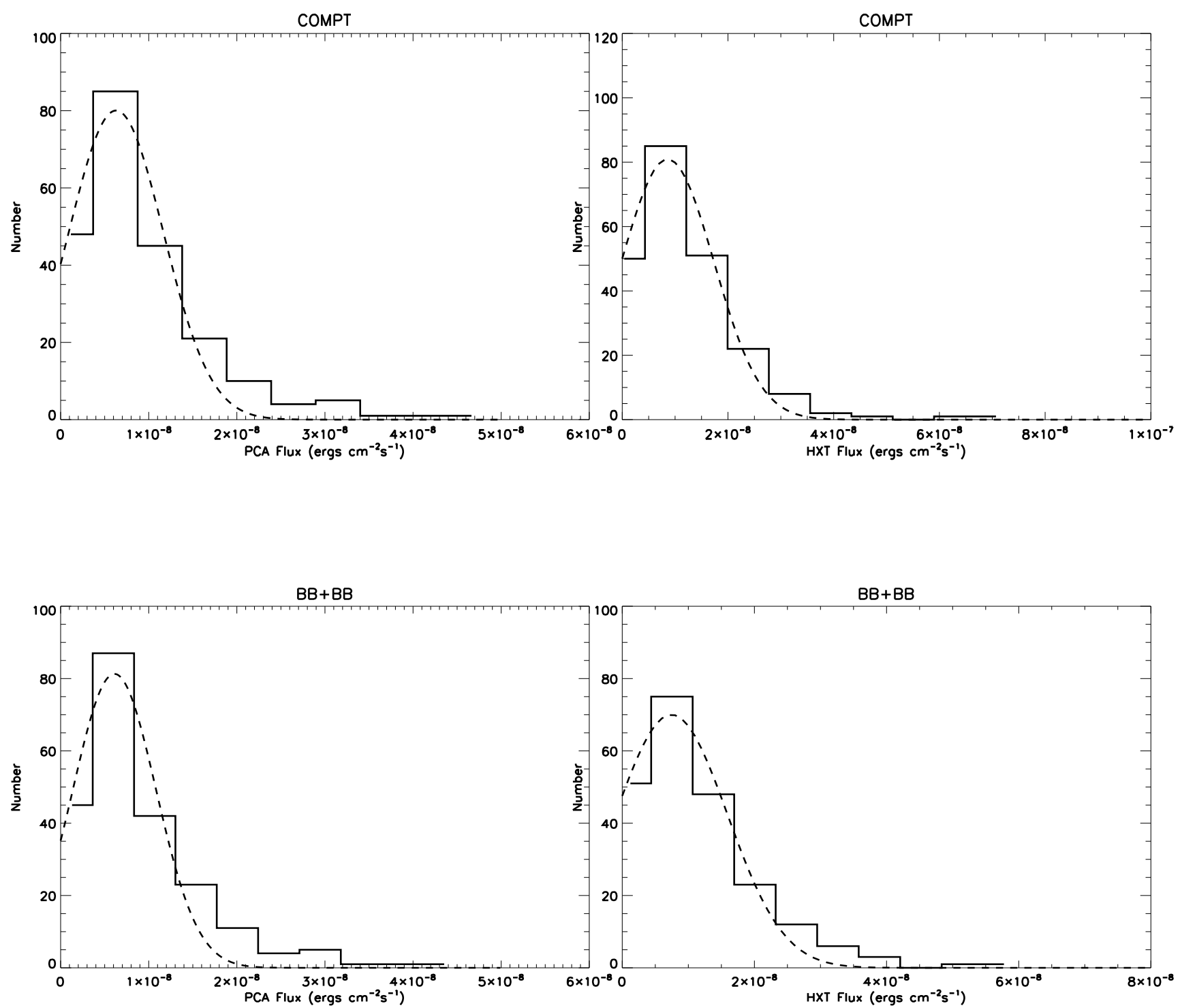

Figure 3.8 SGR 1806-20 Fitted flux distributions for PCA (right) and HEXTE fits (left) for the COMPT (top) and BB+BB (bottom) models. 

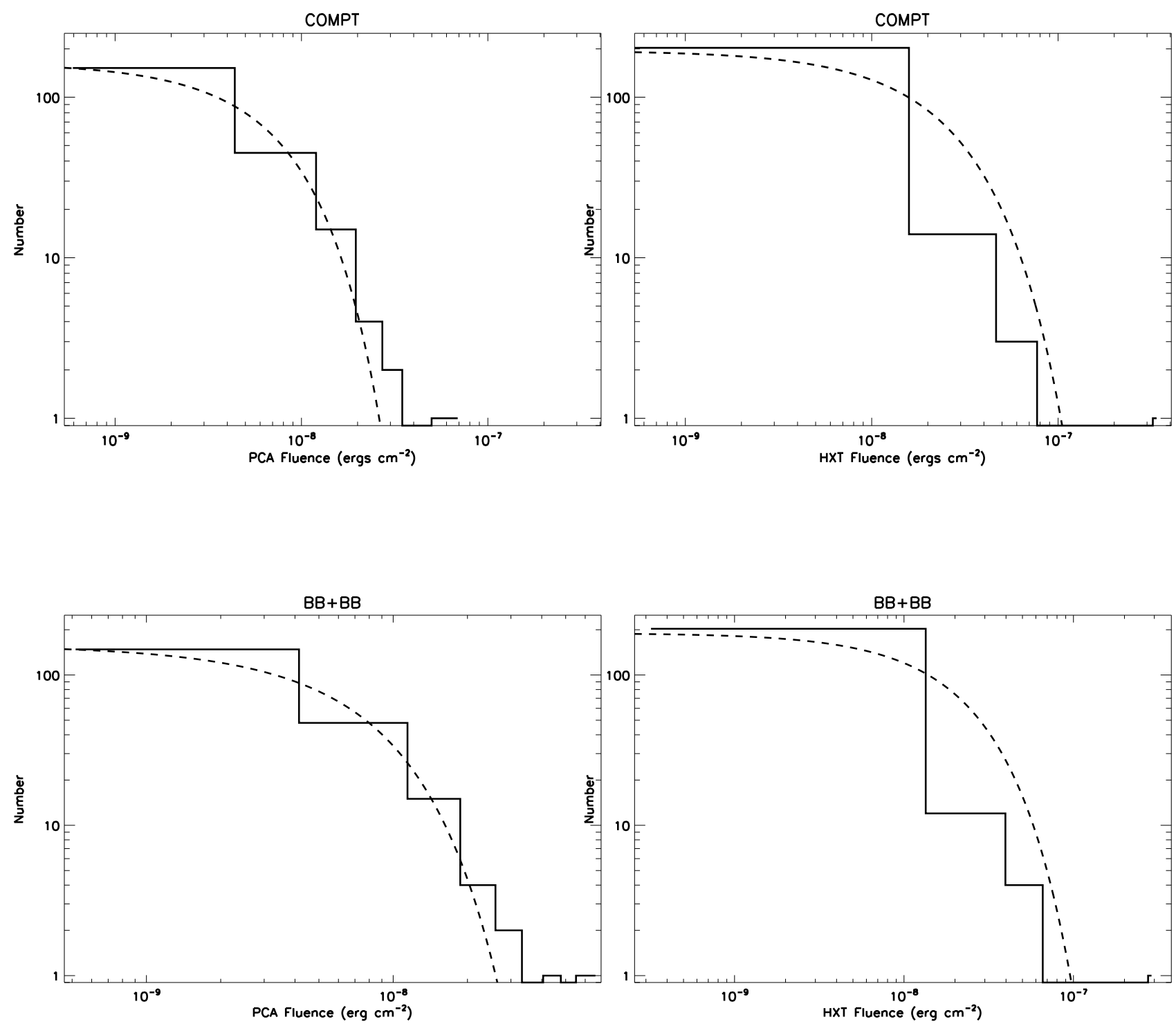

Figure 3.9 SGR 1806-20 Fitted fluence distributions for PCA (right) and HEXTE fits (left) for the COMPT (top) and BB+BB (bottom) models. Gaussian fits are shown with dashed lines. 

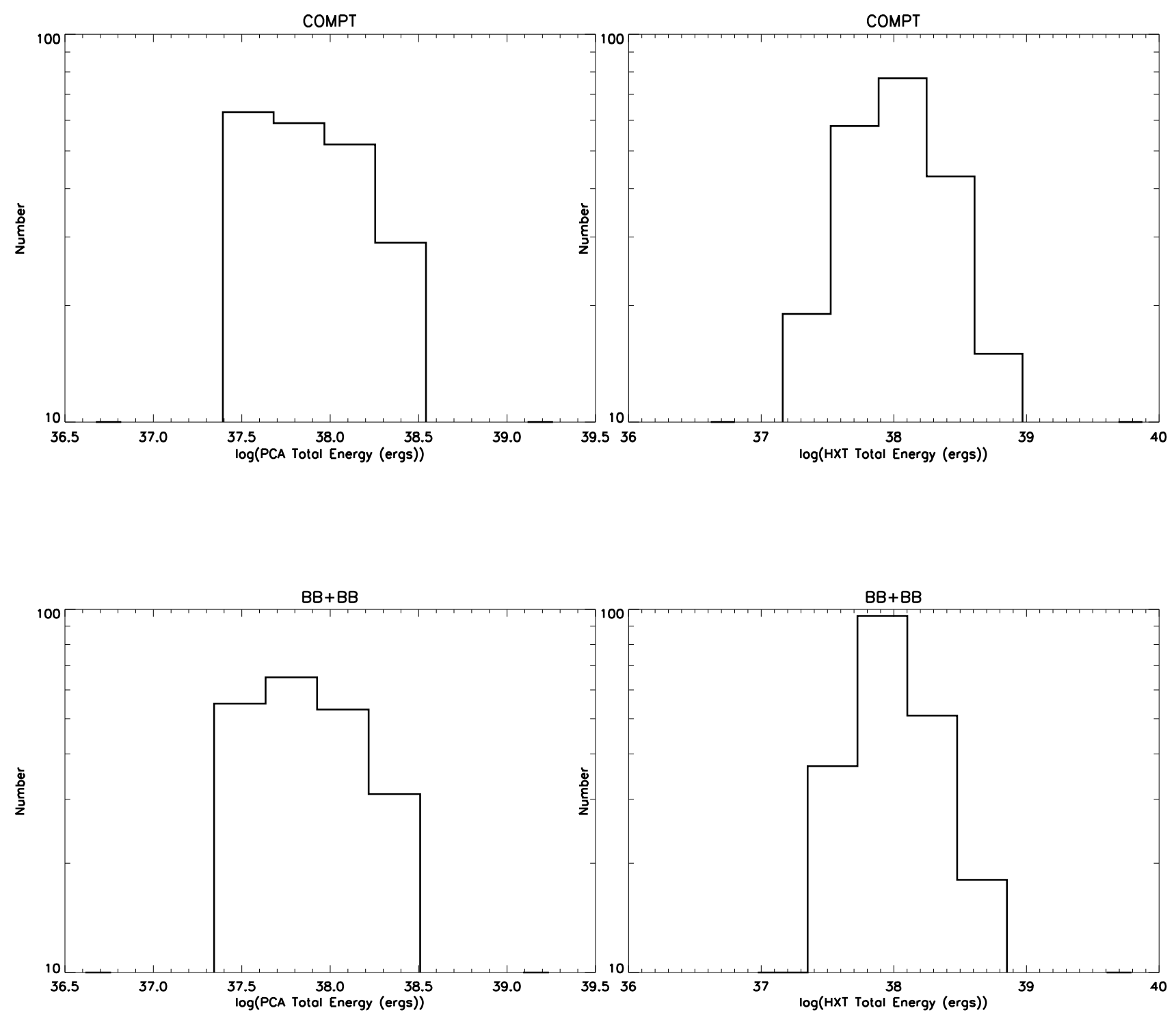

Figure 3.10 SGR 1806-20 Log-log plots of total energy for PCA (right) and HEXTE fits (left) for the COMPT (top) and BB+BB (bottom) models. 


\subsubsection{Detailed Spectral Analysis Results}

We present the joint fit results and $1 \sigma$ errors together with the statistic $\left(\chi^{2}\right)$ and degree of freedom information for all bursts examined in table 3.2 for SGR J1550-5418, table 3.3 for SGR 1900+14 and table 3.4 for SGR 1806-20. In these tables, column 1 is the burst ID in accordance with the observation ID's given in column 1 of Tables 2.1, 2.2 and 2.3. Columns 2-4 correspond to the temperatures of the two additive blackbody components (in $\mathrm{keV}$ ) and the fit statistics of the $\mathrm{BB}+\mathrm{BB}$ model. The temperature (in $\mathrm{keV}$ ) of the blackbody component, the photon index $(\Gamma)$ of the power law component and the fit statistics of the $\mathrm{BB}+\mathrm{PO}$ model are shown in columns 5-7. In columns 8-10 are the temperatures of the two additive blackbody components (in keV) and the fit statistics of the LB+LB model. Columns 13-15 correspond to the cut-off energy $\left(E_{c u t}\right)$, photon index $(\alpha)$, fit statistics and fluxes calculated for PCA and HEXTE for the COMPT model. We report flux values for the COMPT model since it yields lowest reduced $\chi^{2}$ values for most bursts of all three sources. For a more robust statistical comparison of models, see Section 4.1. 


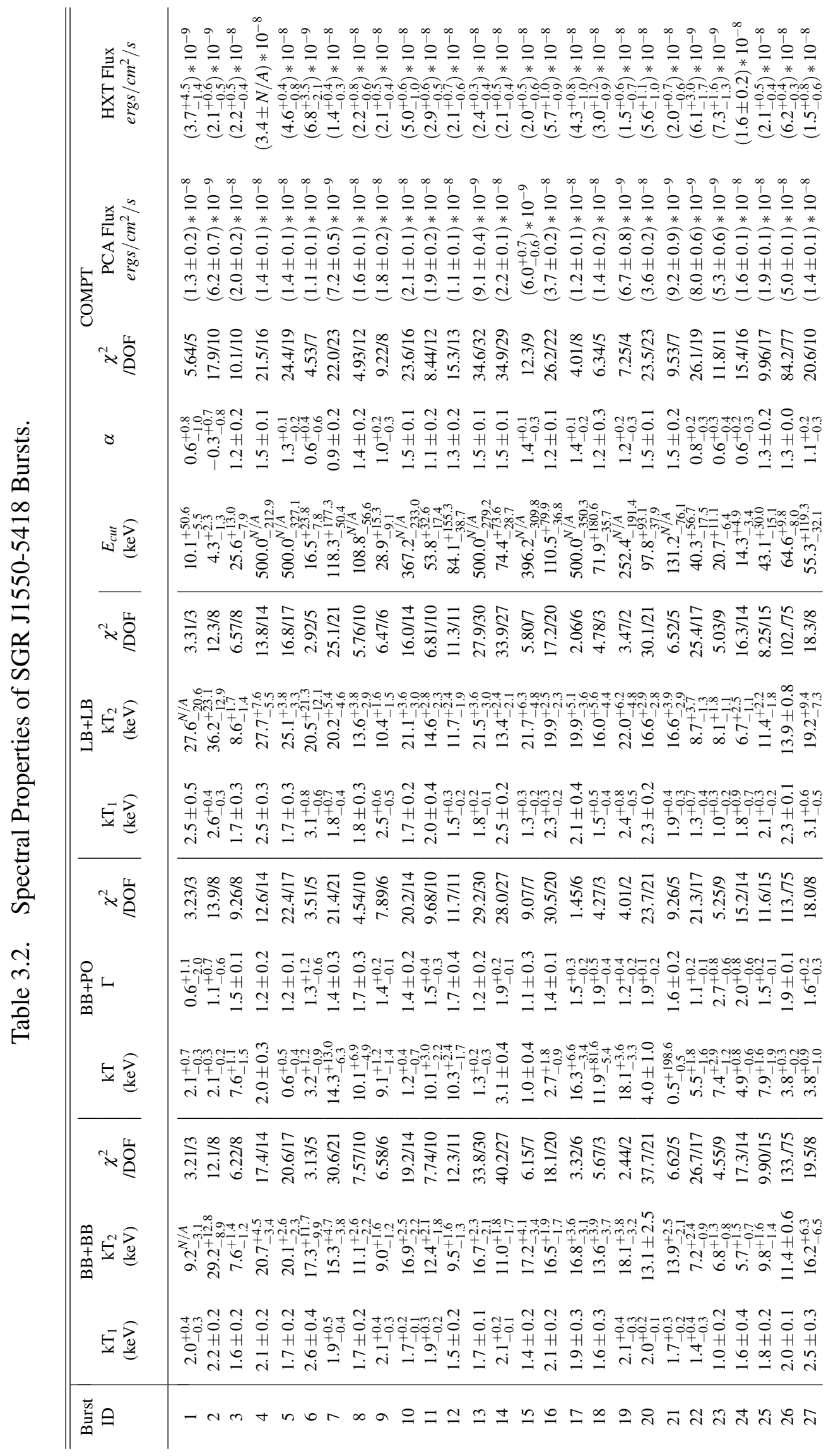




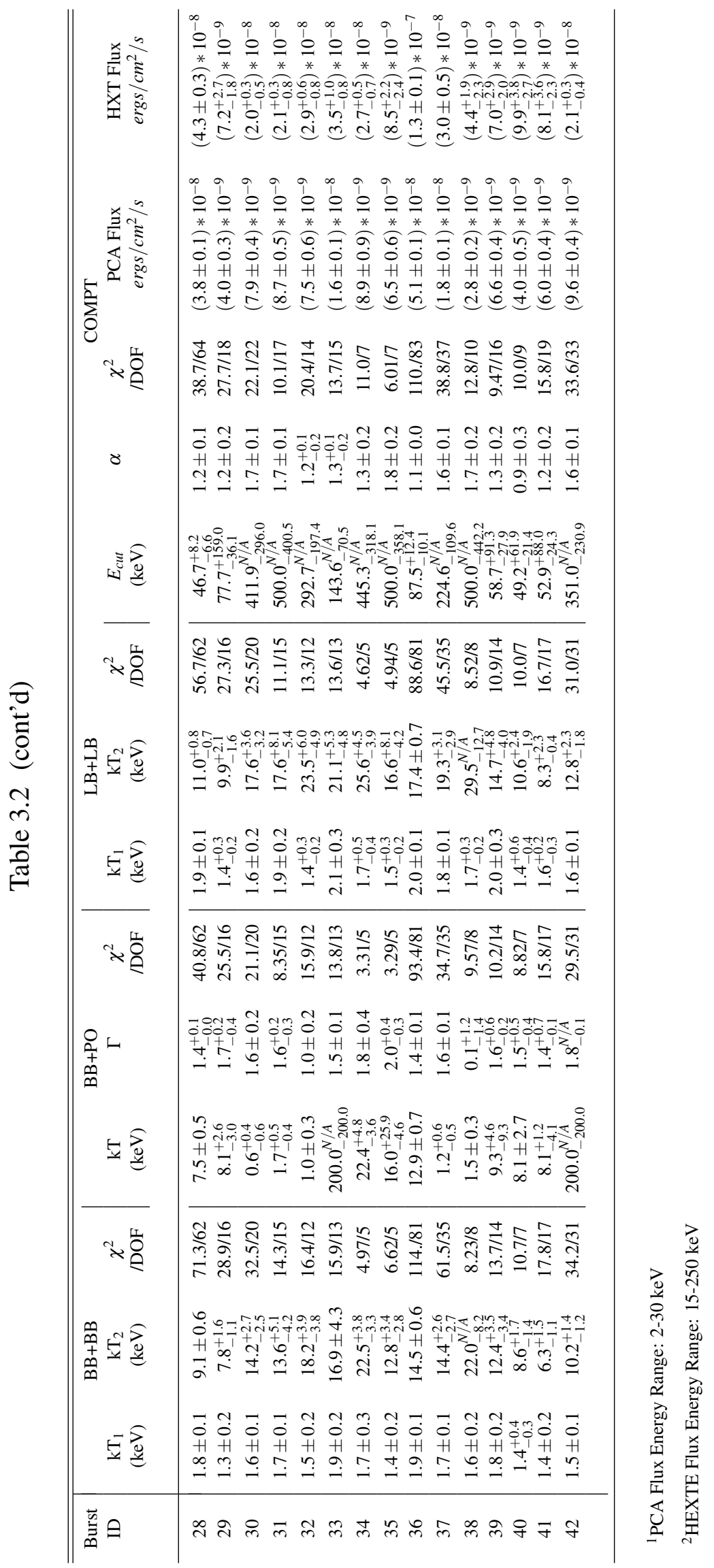




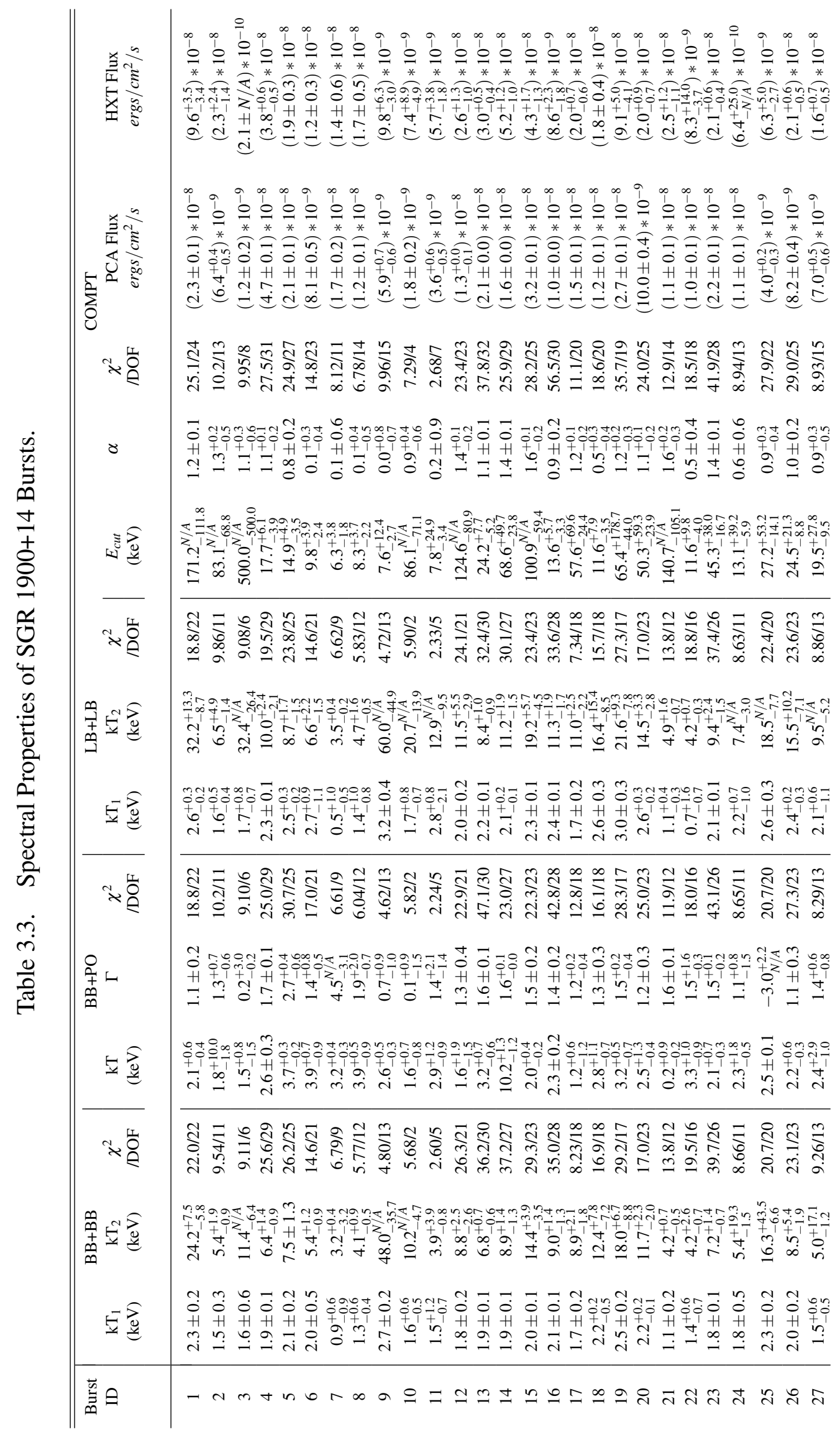




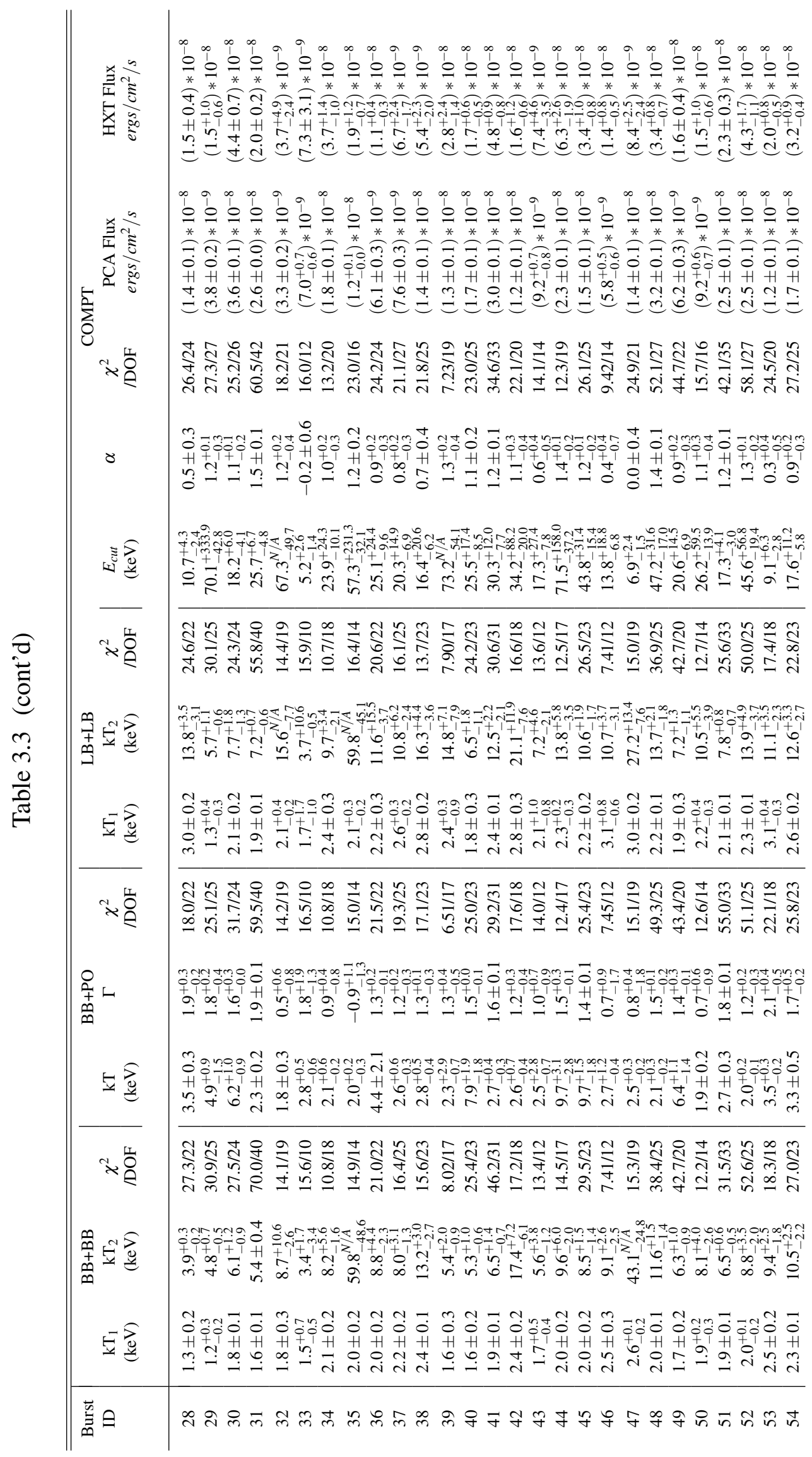




\begin{tabular}{|c|c|}
\hline 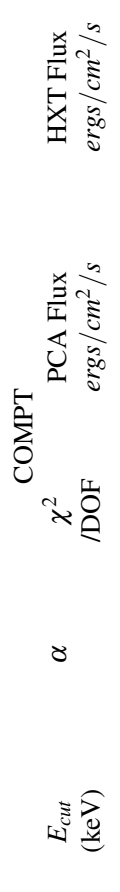 & 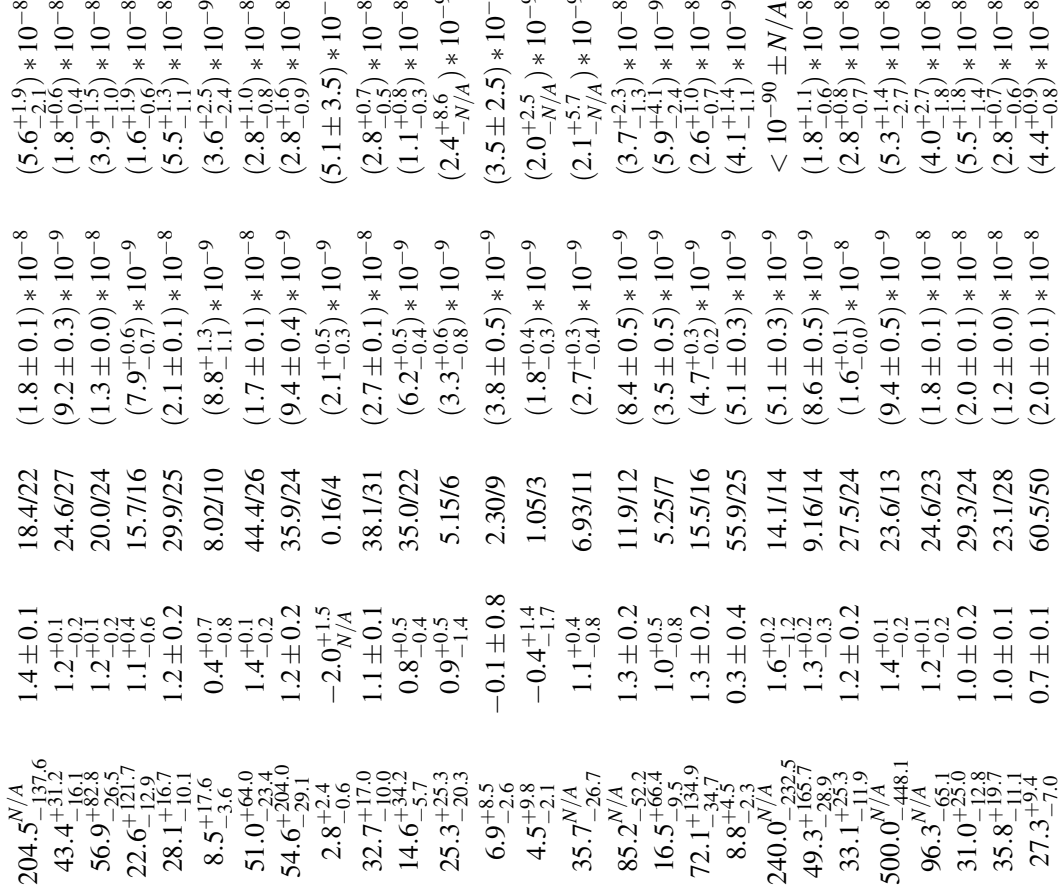 \\
\hline 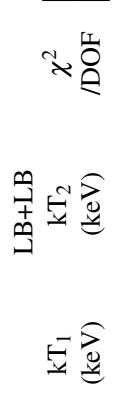 & 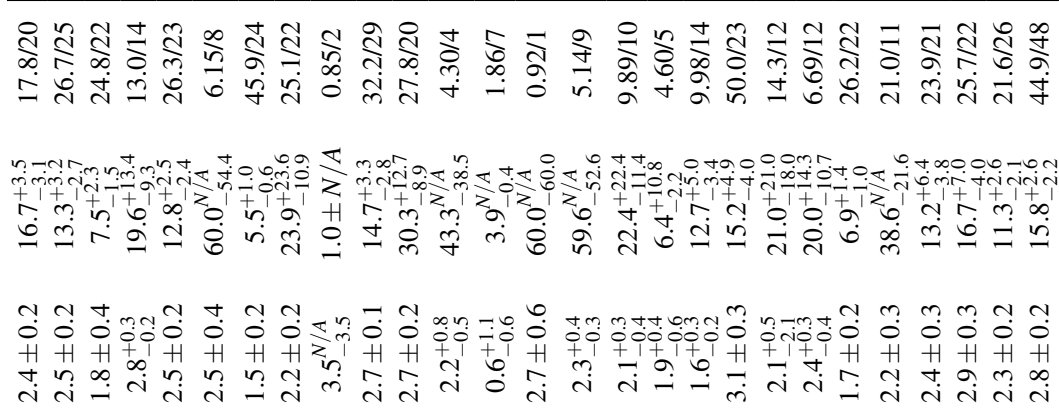 \\
\hline 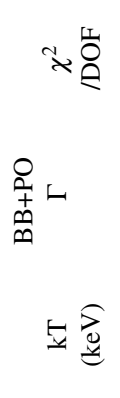 & 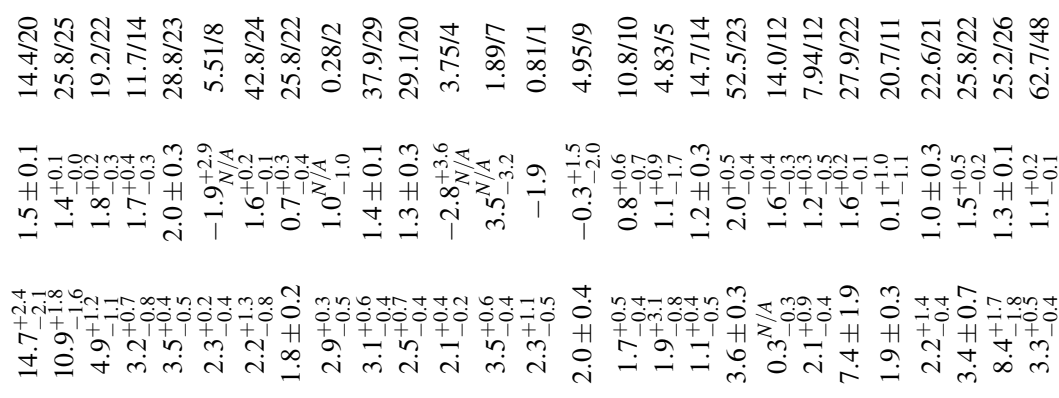 \\
\hline 焉事 & 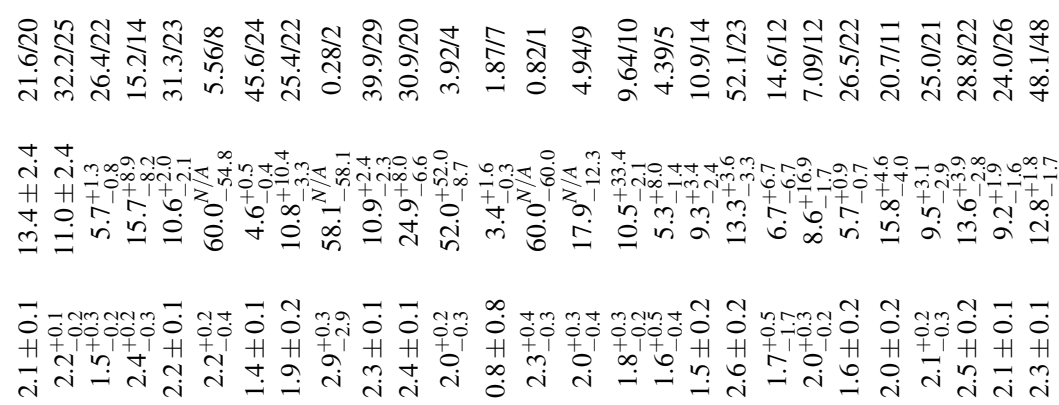 \\
\hline$\stackrel{\vec{m}}{=}$ & 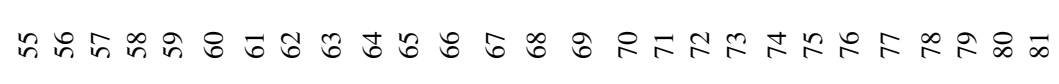 \\
\hline
\end{tabular}




\begin{tabular}{|c|c|}
\hline 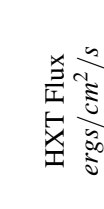 & 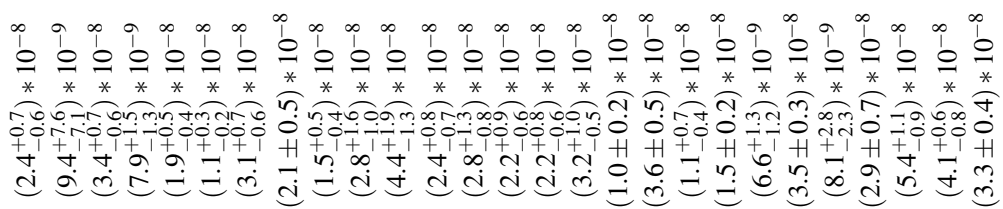 \\
\hline 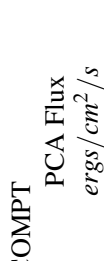 & 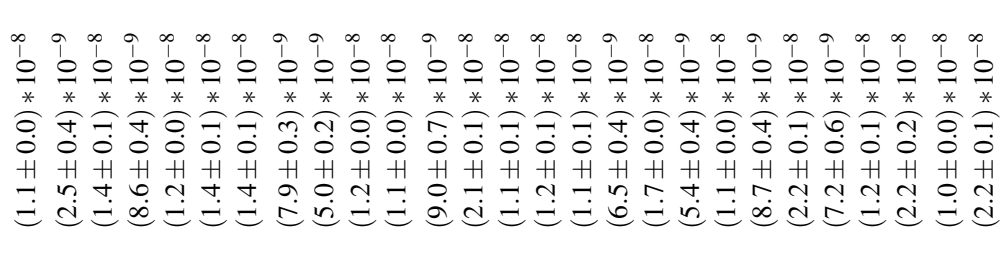 \\
\hline 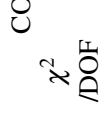 & 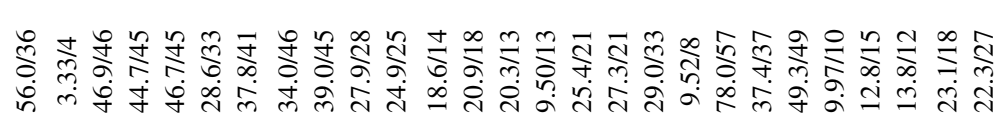 \\
\hline ૪ & 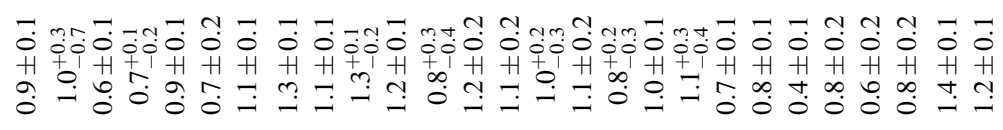 \\
\hline 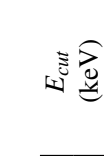 & 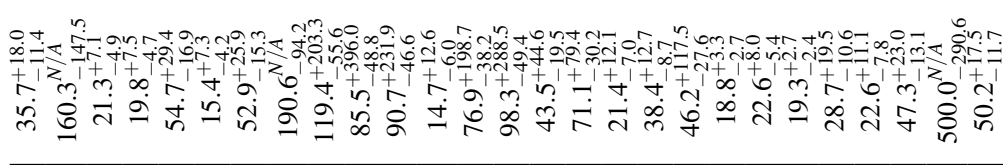 \\
\hline$\underset{\overbrace{}}{0}$ & 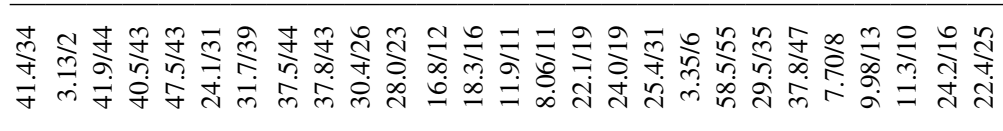 \\
\hline & 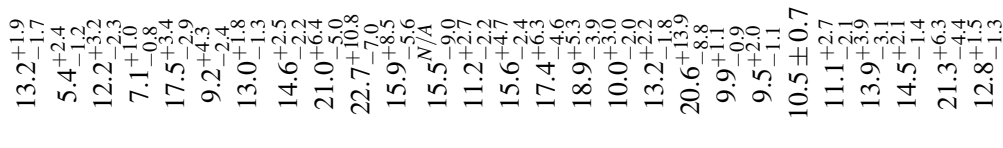 \\
\hline$E$ & 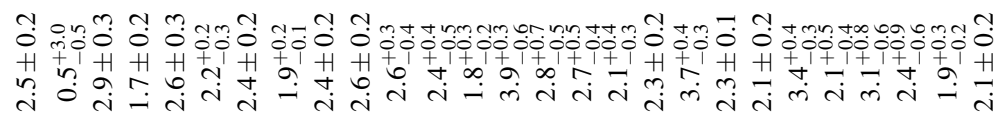 \\
\hline & 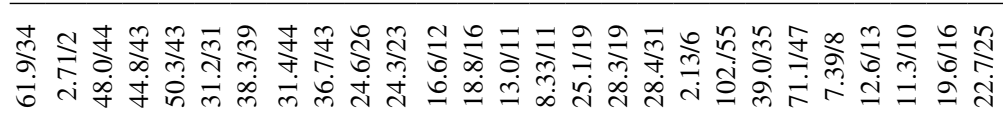 \\
\hline 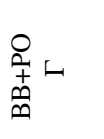 & 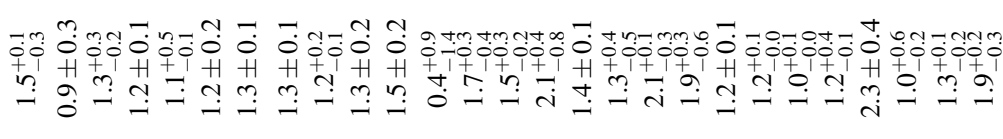 \\
\hline$\underline{5}$ & 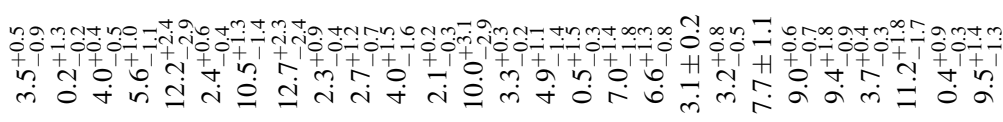 \\
\hline$\approx \stackrel{0}{0}$ & 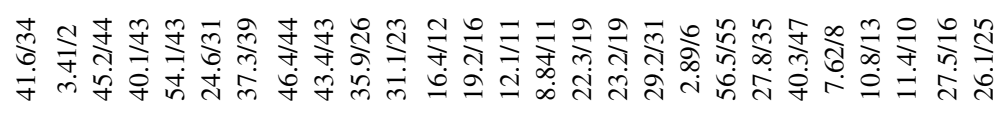 \\
\hline 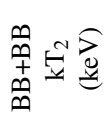 & 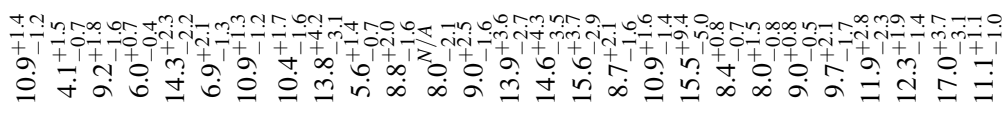 \\
\hline$E \overline{8}$ & 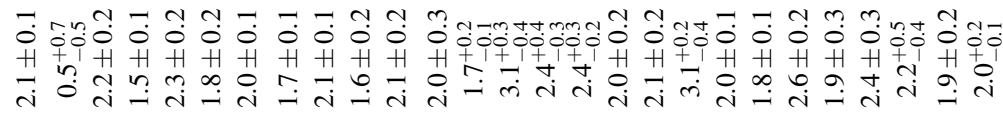 \\
\hline 䓂目 & 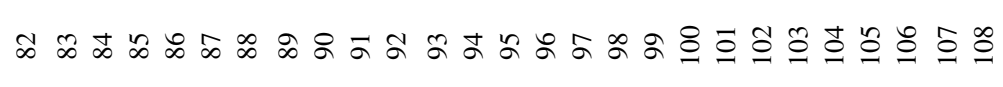 \\
\hline
\end{tabular}




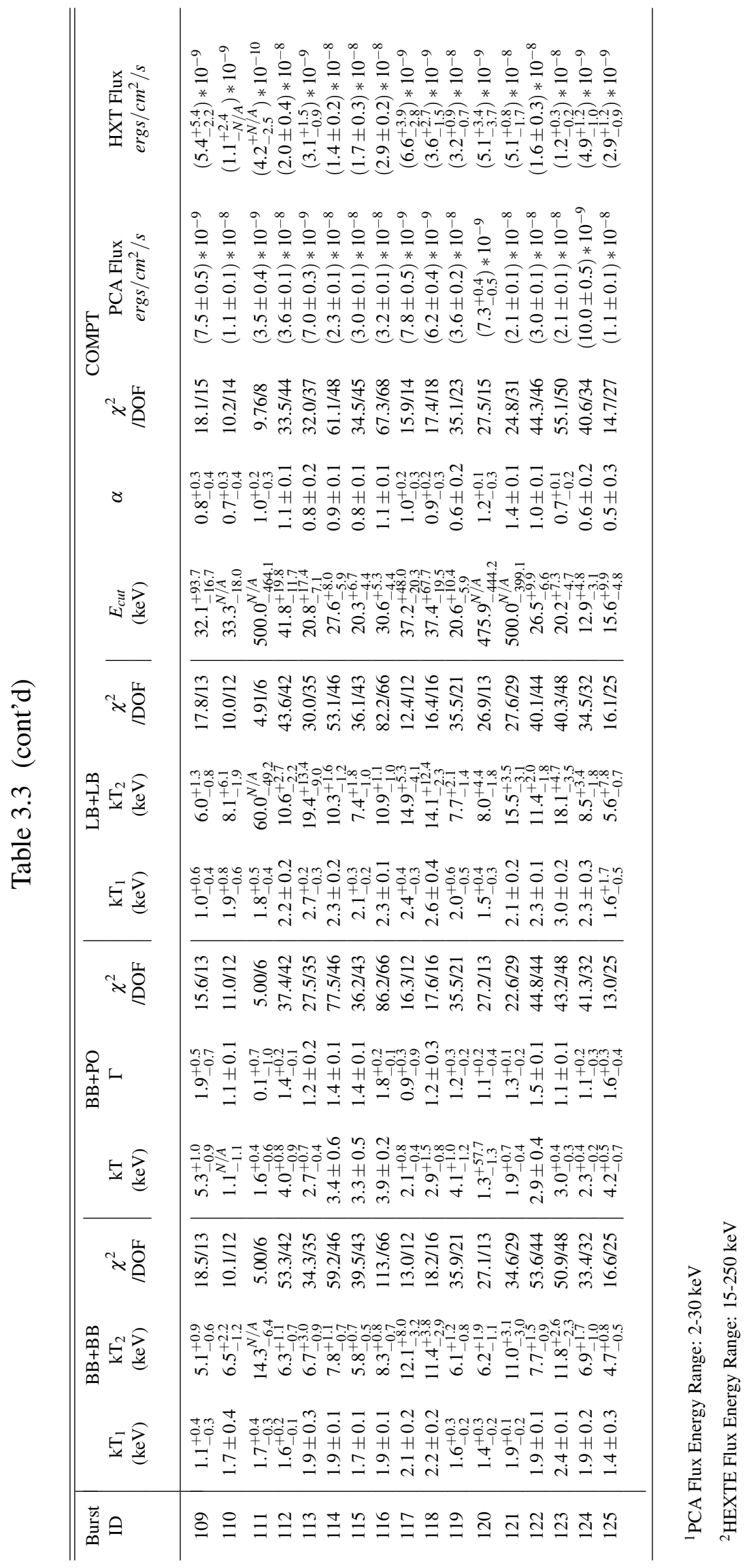




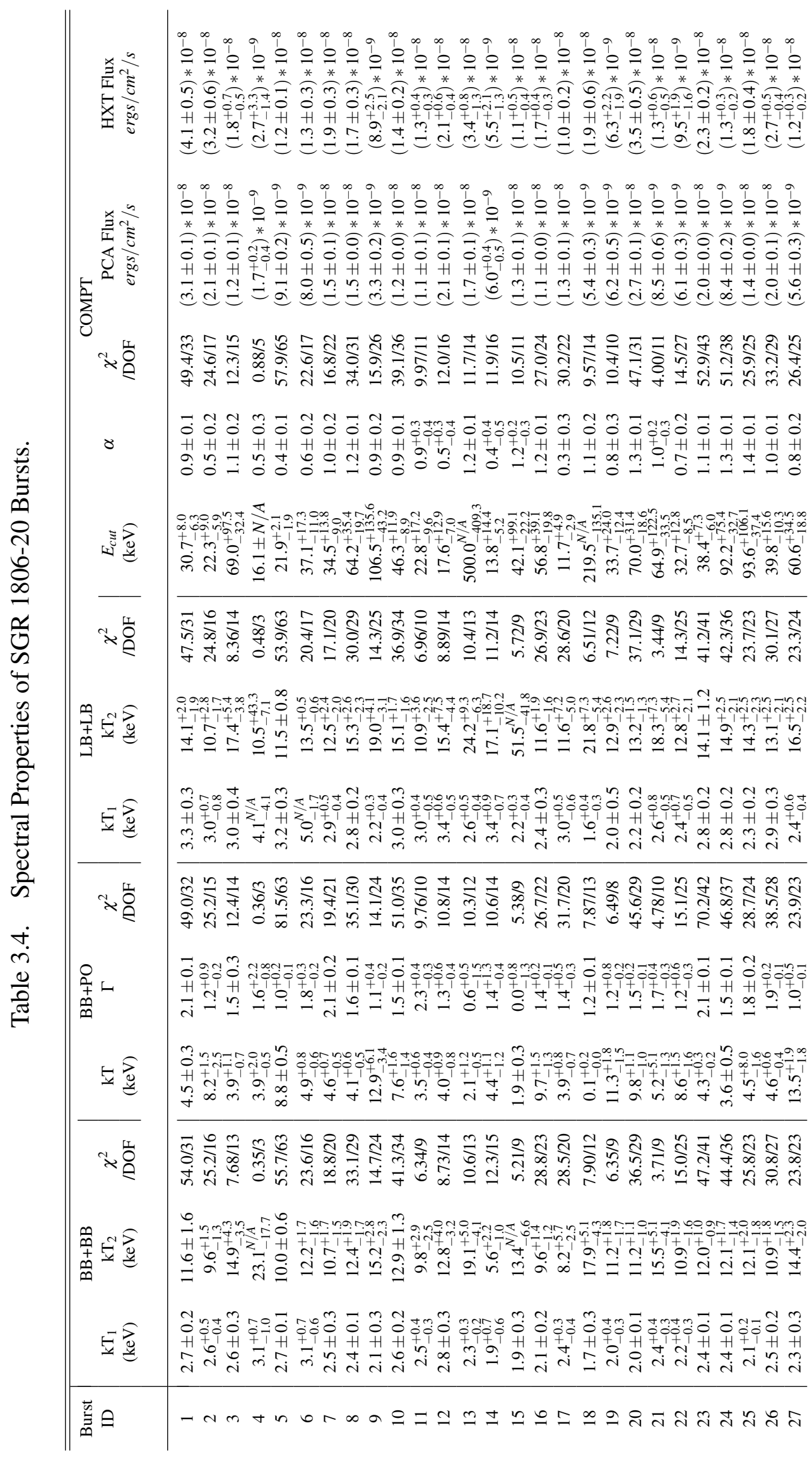




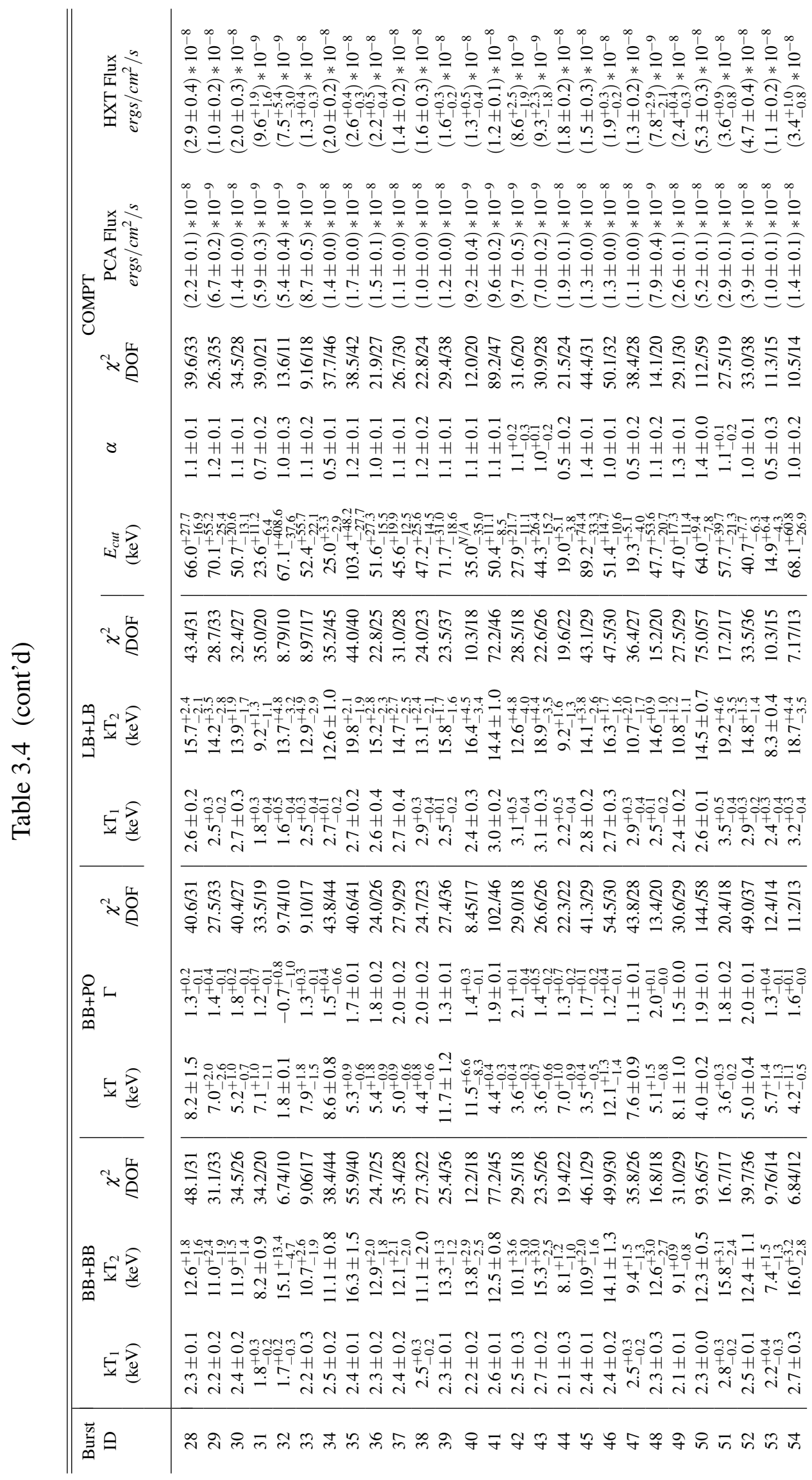




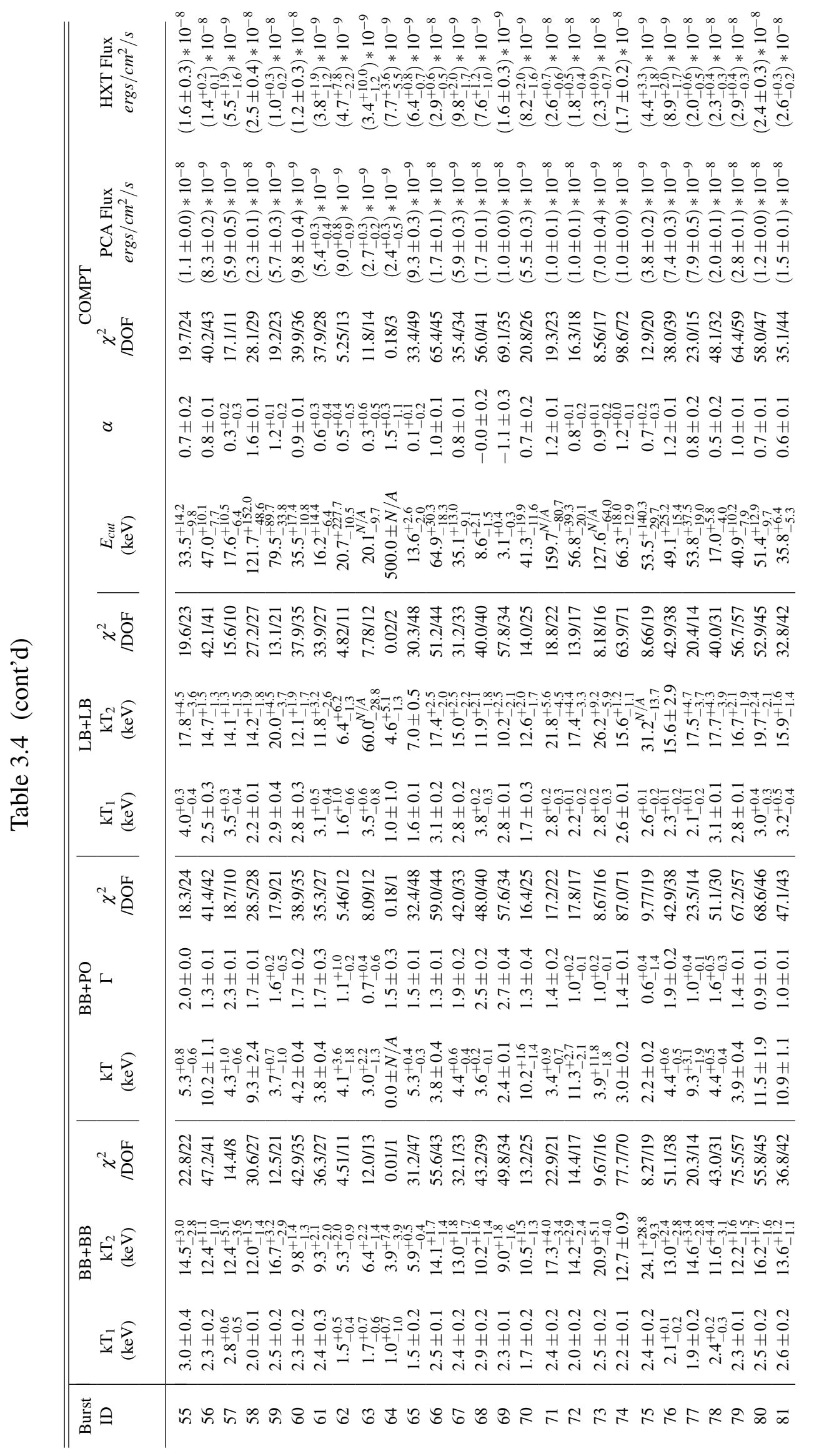




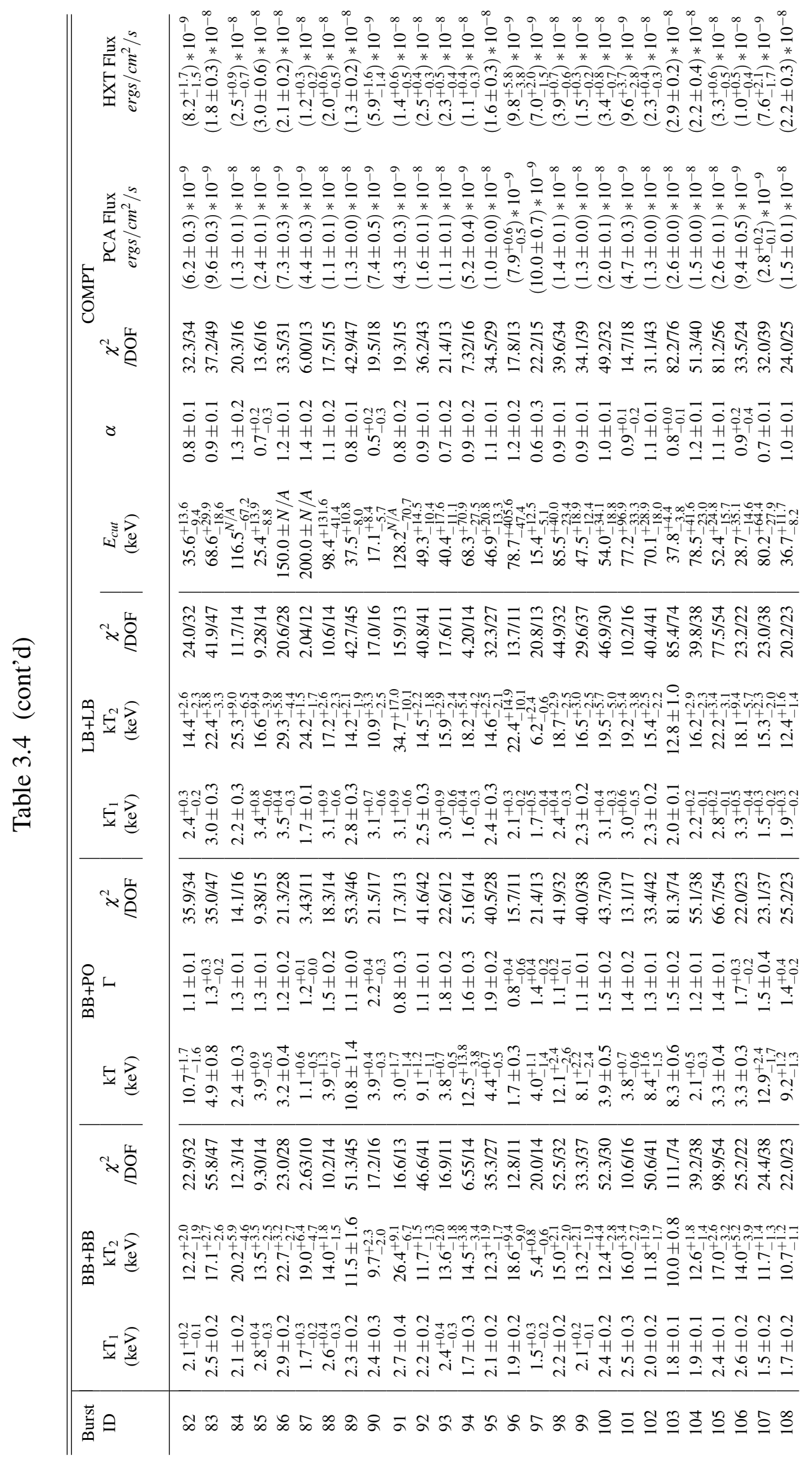




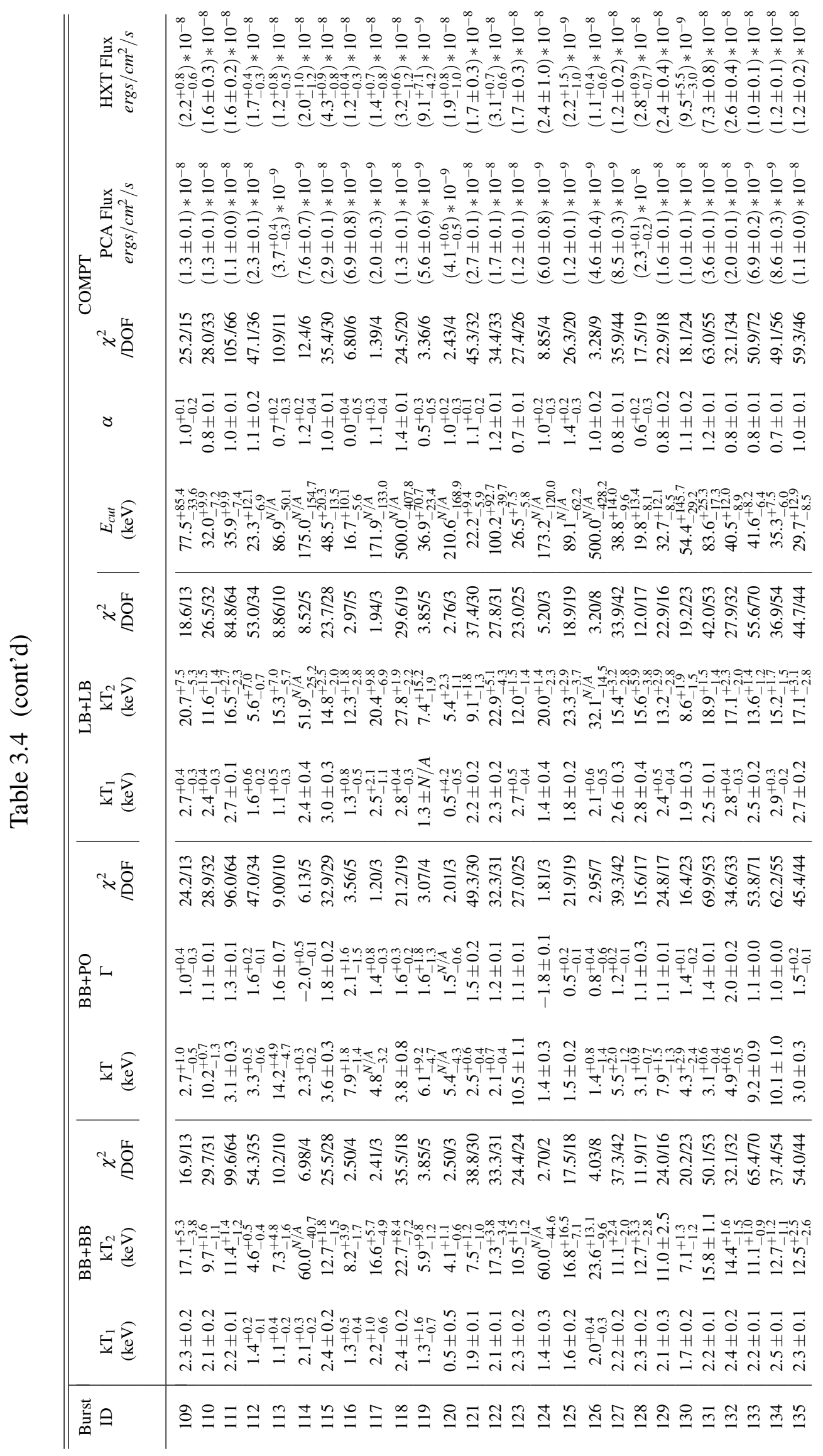




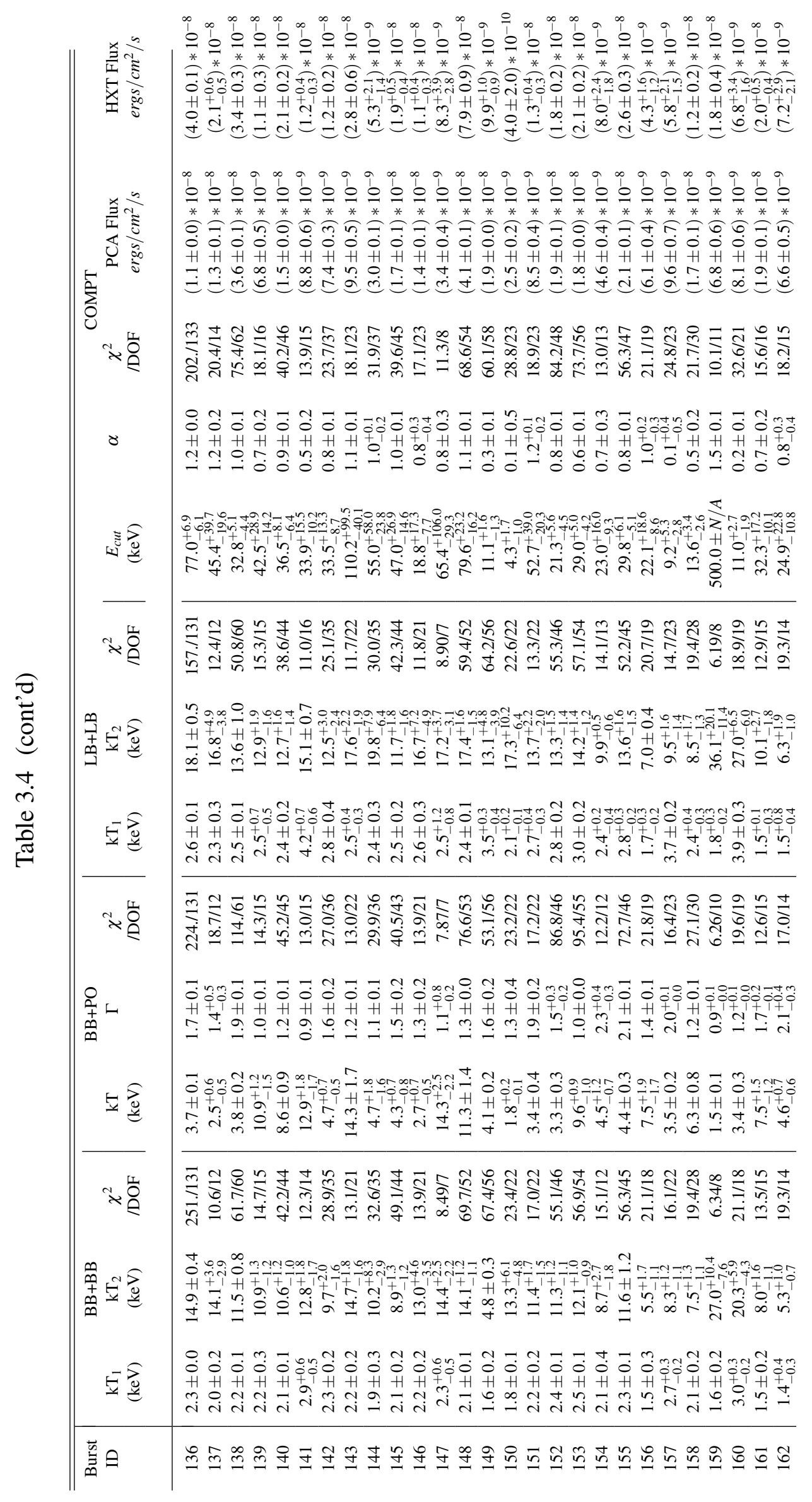




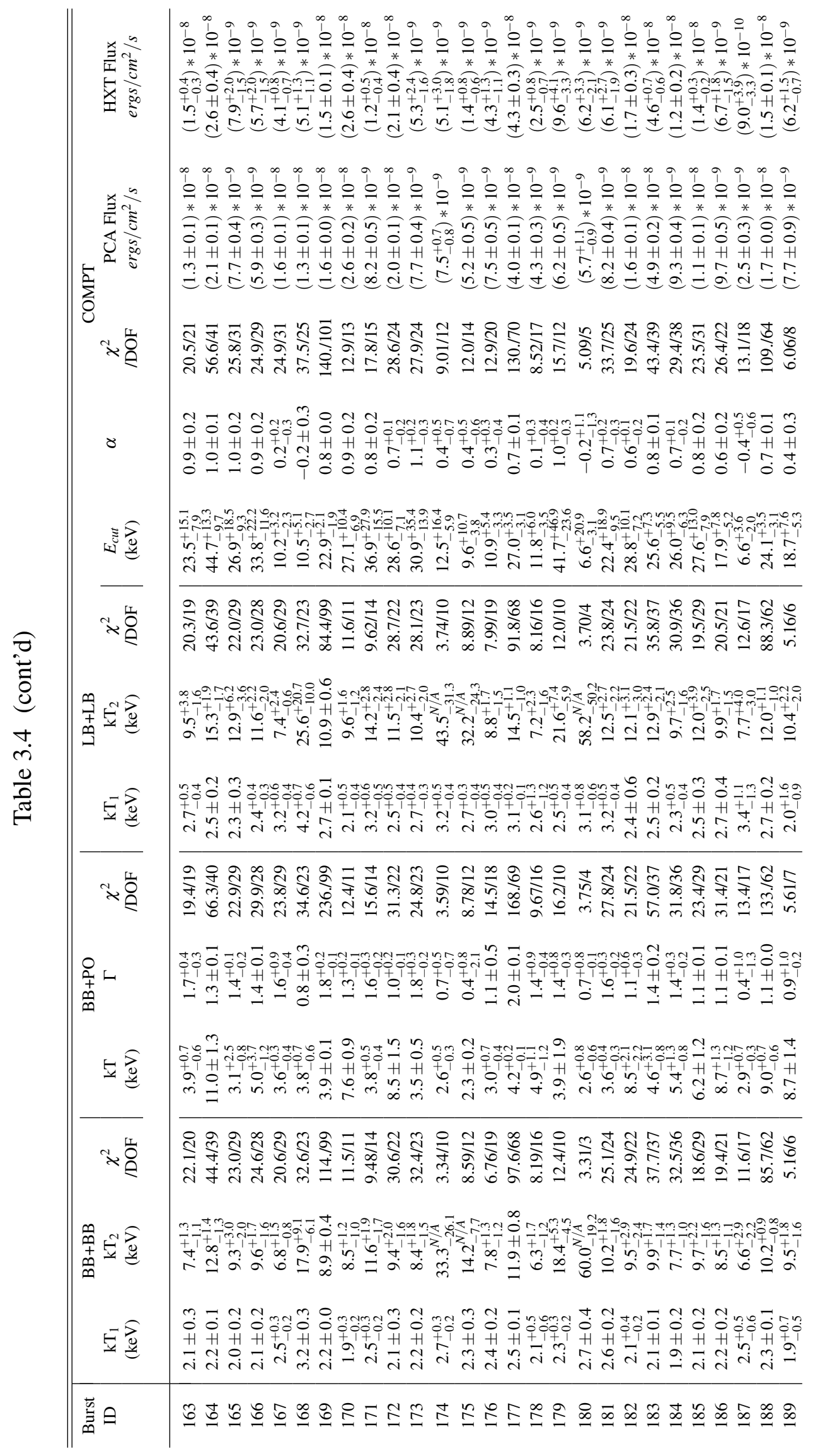




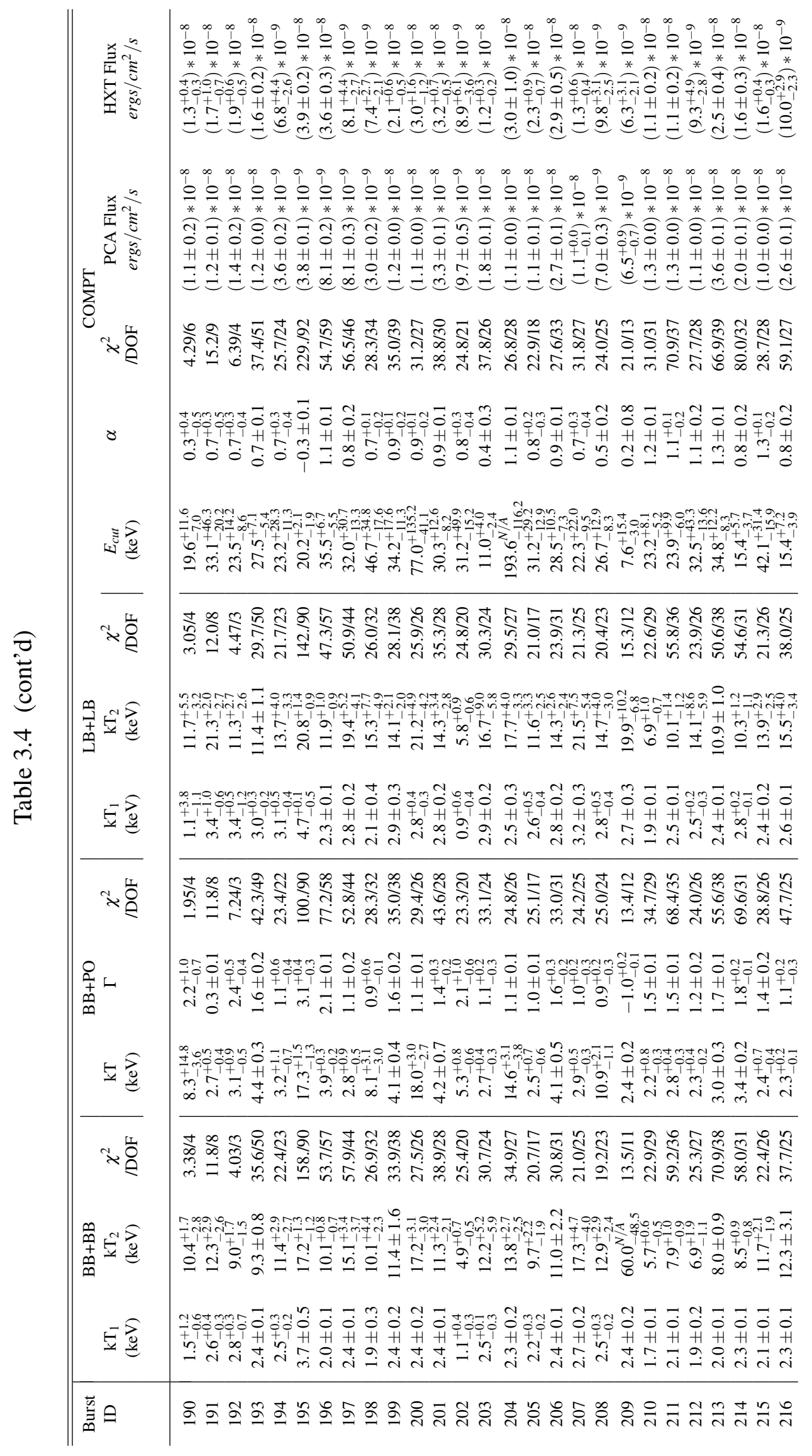




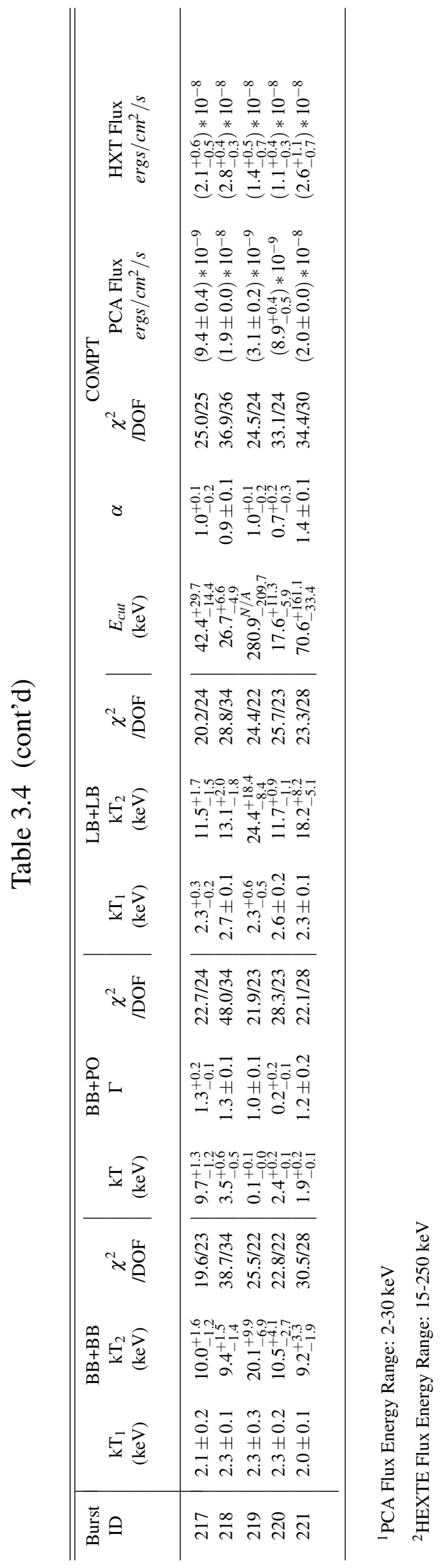




\subsection{Peak Energy Comparison with Previous Studies}

We used the Cutoffpl model (referred above as COMPT) on XSPEC to find distributions for the high energy cut parameter. A different parametrization of the same Comptonized emission mechanism has been used in previous studies (e.g., Lin et al. 2012; van der Horst et al. 2012; Feroci et al. 2004) to analyze bursts from some sources in this study. We will henceforth refer to this parametrization as the COMPT2 model

The COMPT2 model is in a single power law shape with a peak energy expressed as:

$$
f=A \exp \left[-E(2+\lambda) / E_{\text {peak }}\right]\left(E / E_{\text {piv }}\right)^{\lambda}
$$

where, $\mathrm{f}$ is the photon flux in photons $\mathrm{cm}^{-1} \mathrm{~s}^{-2} \mathrm{keV}^{-1}, \mathrm{~A}$ is the amplitude with units same as $\mathrm{f}, E_{\text {peak }}$ is the energy (in $\mathrm{keV}$ ) at which the spectral distribution function makes its peak, $\lambda$ is the photon index (defined as $-\alpha$ where $\alpha$ is the photon index of the COMPT model), and $E_{p i v}$ is the pivot energy fixed at a certain value (20 keV, Lin et al. 2012).

The high energy cut parameter in the COMPT model can be mapped to the peak energy parameter of the COMPT2 model, used previously by Lin et al. 2012, van der Horst et al. 2012 and Feroci et al. 2004 by equating the two fluxes where negative of the photon index $(-\alpha)$ of the COMPT model is mapped to the photon index $(\lambda)$ of the COMPT2 model yielding the following simple formula:

$$
E_{\text {peak }}=(2-\alpha) \times E_{\text {cut }}
$$

We considered the possibility that using a different parametrization in spectral analysis may result in different fits due to local minima. We re-employed our analysis using the COMPT2 model on a sample spectra (20 bursts) and found the same fit statistics (i.e. same minima) with the previously discussed mapping from $E_{c u t}$ to $E_{\text {peak }}$ holding true within errors. Therefore, we used the above method to map our resulting high energy cut using the fitted photon index values to find the peak energy values for comparison.

In Figure 3.11, we report the distributions of peak energies of SGR 1900+14 and SGR 1806-20 including only fits with well-constrained parameters. The gaussian mean of $17.23 \pm 1.42$ for SGR 1900+14 agrees within errors with Feroci et al. 2004's gaussian mean of $15.8 \pm 2.3$ conducted in the energy range 1.5-100 keV. SGR J1550-5418 does not have large enough sample burst size to provide a reliable distribution, therefore we report the average values of fit results with wellconstrained parameters. The average peak energy calculated for SGR J1550-5418 is $44.59 \mathrm{keV}$, with a minimum of $20.46 \mathrm{keV}$ and a maximum of $77.04 \mathrm{keV}$, agreeing within errors with those found by Lin et al. $2012(39 \pm 13 \mathrm{keV})$ and van der Horst et al. $2012(45 \pm 2.1 \mathrm{keV})$. The gaussian mean of peak energy for SGR 1806-20 is $32.02 \pm 1.84 \mathrm{keV}$. 

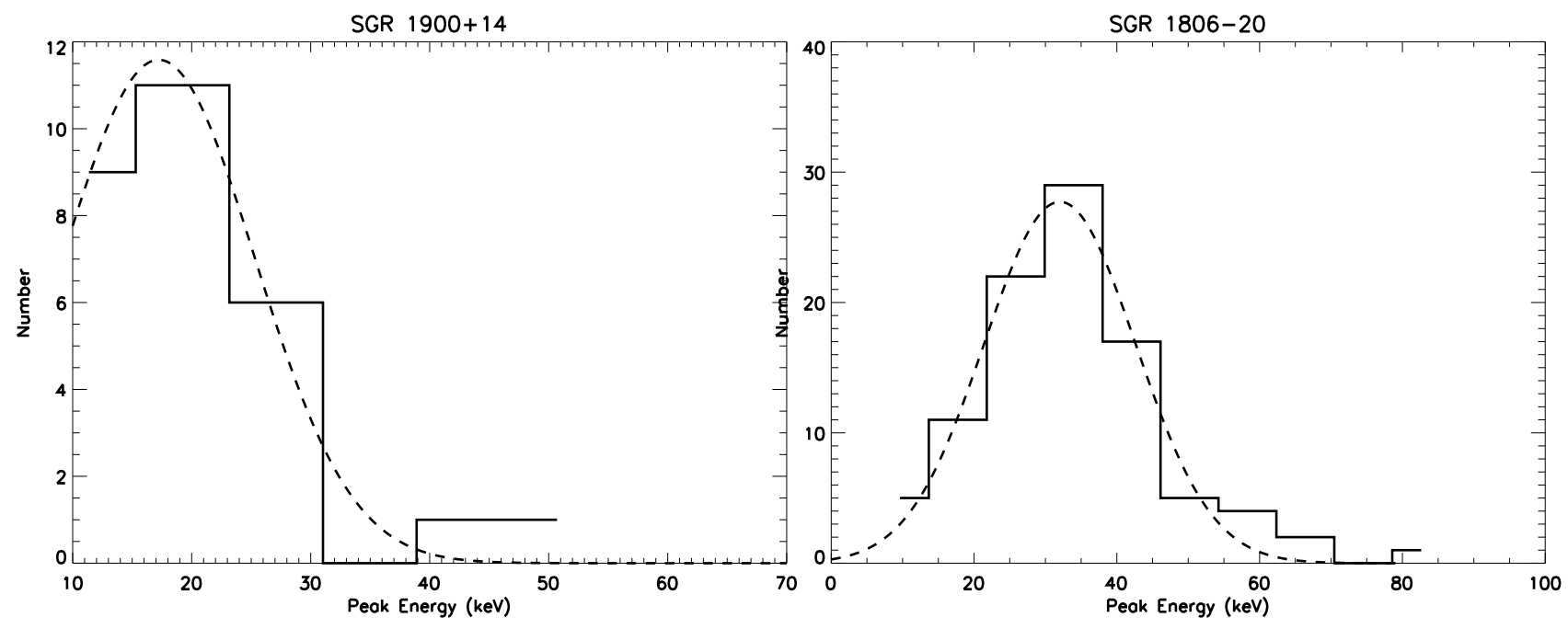

Figure 3.11 SGR 1900+14 (left) and SGR 1806-20 (right) Peak Energy Distributions. 


\section{Chapter 4}

\section{Discussion}

\subsection{Identification of the Best Describing Model}

Even though one of the four models that were employed to fit the broadband X-ray spectra of magnetar bursts yield the minimum reduced $\chi^{2}$ value, it is statistically not possible to disregard the alternatives simply by a $\Delta \chi^{2}$ test. This issue becomes more complicated given the fact that the COMPT model involves one less free parameter than thermal models, resulting in, on average, one more degrees of freedom. The additional degree of freedom enhances the fitting power of COMPT in the cases where the spectrum could be easily represented by two or more models. In such cases, competing models can be better compared by simulations based on fit results.

\subsubsection{Simulation Procedure}

To compare burst emission models, we performed extensive simulations for each burst as follows. Overall, the COMPT model performs the best in fitting magnetar burst spectra, as discussed before. Therefore, we took the COMPT model as the null hypothesis (i.e. seed model) and generated 1000 spectra using the resulting COMPT fit parameters for each burst whose COMPT model parameters were somehow constrained (parameter error less than $50 \%$ of the parameter). As an alternative hypothesis (test model), we selected one of the three thermal models, whose reduced $\chi^{2}$ value was the smallest. Note that the remaining models have equal number of degrees of freedom and a simple $\Delta \chi^{2}$ test is applicable for comparison.

When the test model did not provide well-constrained parameters (i.e. less than 50\% errors), we have selected the next model with the least reduced $\chi^{2}$ value to be the test model. If none of the test models provided well-constrained parameters, we discontinued the simulation for that event. We found that four out of 42 events examined for SGR J1550-5418, 21 out of 125 events examined for SGR 1900+14 and 77 out of 221 bursts from SGR 1806-20 provided such well-constrained 
parameters for seed and test models, and were included in our simulations. We have then fit the generated 1000 spectra for each burst with the COMPT and test models.

We used a significance level of 0.05 (i.e. $\alpha=0.05$ ) for each burst included in the simulation. For a $\chi^{2}$ distribution with $\mathrm{df}=1$ (since the degrees of freedom on the test and seed model differ by one in each case), this corresponds to a $\Delta \chi^{2}$ value of 3.84. Therefore, we defined our rejection region of the null hypothesis (i.e. when we accept the test model) as the region where the test model $\chi^{2}$ is less than the seed model $\chi^{2}$ by at least 3.84. We hypothesize that, if a truly Comptonized spectrum in fact provides better non-reduced fit statistics within $\alpha<0.05$ significance, then our fit results where COMPT provides lower $\chi^{2}$ values indicates the true emission mechanism most likely is Comptonized rather than thermal emission.

To check whether the simulation procedure forms a bias towards COMPT, we repeated the same procedure with $\mathrm{BB}+\mathrm{BB}$ as the null hypothesis (seed model) for one event with COMPT as the alternative hypothesis (test model). In this reverse simulation scenario, we similarly defined our rejection region for the null hypothesis as when COMPT $\chi^{2}$ value was less than BB+BB $\chi^{2}$ by at least $\chi_{0.05,1}^{2}=3.84$. BB+BB model was accepted in $100 \%$ of trials when it acted as the seed model. COMPT model was accepted in $99.7 \%$ of trials when COMPT was the seed model in the original simulation for the same event. By comparing these results, we concluded that the simulation procedure accepts the inherent emission mechanism within the level of significance with no bias towards any model. Therefore, we continued the simulations with COMPT as the seed model since our spectral analysis results show the majority of bursts are better fit with the COMPT model.

Following the procedure by Lin et al. 2012 we define our p-value to be the fraction of simulated spectra better fitted by COMPT model within 0.05 significance. If the p-value exceeds 0.9 , we conclude that the COMPT model provides better fit statistics than the test model when it is the underlying emission mechanism.

\subsubsection{Results}

Overall, COMPT is the most frequently (in $67.6 \%$ of bursts) preferred model based on simulation results (i.e. COMPT provides significantly better fit statistics in more than $90 \%$ of trials for $16 / 19$ events compared to the $\mathrm{BB}+\mathrm{PO}$ model, 12/17 events compared to the $\mathrm{BB}+\mathrm{BB}$ model and 41/66 events compared to the LB+LB model).

Our spectral analysis results show that the majority of bursts are better fit by COMPT. The simulation results suggest that when the underlying emission mechanism is described by COMPT model, COMPT provides better fit statistics in most cases. As a result, the combined spectral analysis and simulation results suggest COMPT model as the model that describes the emission 


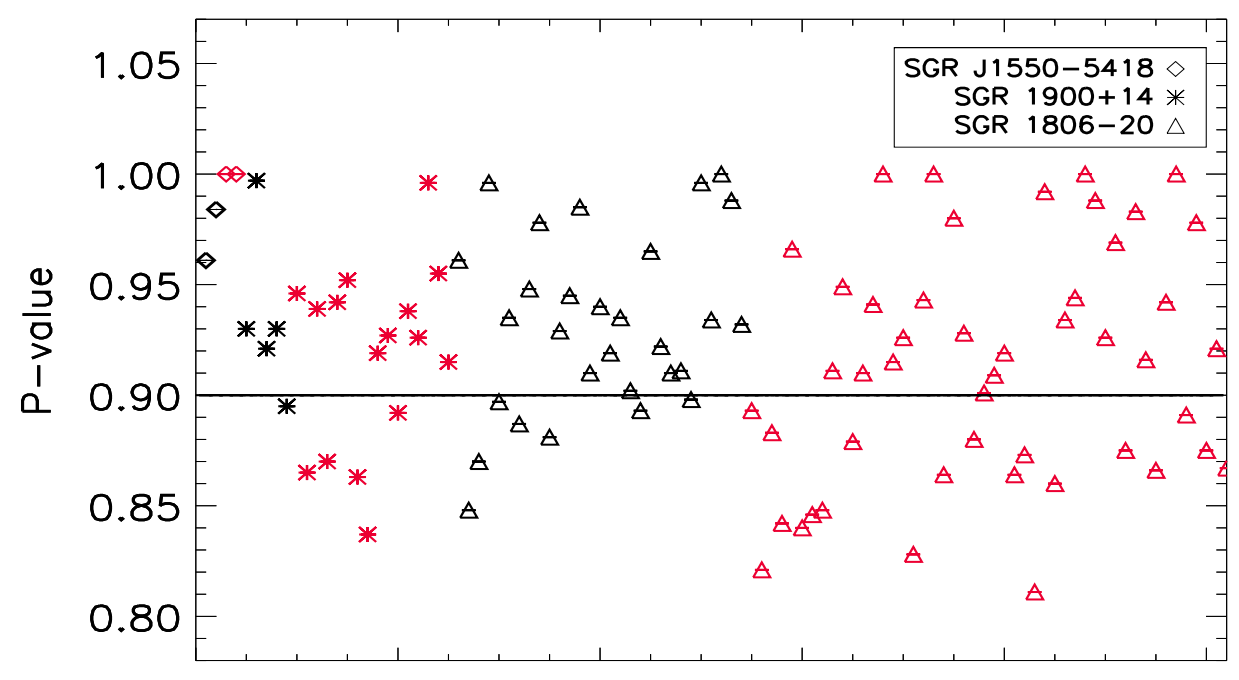

Figure 4.1 Visual representation of resulting p-values. P-value above 0.9 indicates that COMPT model describes simulated spectra best. Bursts where LB+LB model was selected as the best describing model within thermal models are shown in red.

spectra best for the majority of events.

On the other hand, LB+LB emerges as the best fitting model within the remaining thermal models, providing better fits than $\mathrm{BB}+\mathrm{BB}$ and $\mathrm{BB}+\mathrm{PO}$ models for the majority (66 out of 102) of events (See Figure 4.1 for a visual representation of simulation results). It is important to reinstate that it is possible to compare the thermal models with a simple $\Delta \chi^{2}$ test since these models have the same degrees of freedom. For the remaining models that are not well described by non-thermal emission (i.e. COMPT), our results suggest $\mathrm{LB}+\mathrm{LB}$ model would describe spectra best, compared to $\mathrm{BB}+\mathrm{PO}$ and $\mathrm{BB}+\mathrm{BB}$ models.

In Figure 4.2, we present the distributions for the difference between seed model (COMPT) $\chi^{2}$ and test model $\chi^{2}$ for three events of SGR 1806-20 with different test models for a visual description of simulation results. Our rejection region of COMPT model (null hypothesis) is the area above $C O M P T_{\chi^{2}}$ Test Model $_{\chi^{2}}=3.84$

corresponding to $\alpha=0.05$ significance level. By comparing the areas above and below $\mathrm{x}=3.84$ , and the p-values in the table, it can be reconfirmed that the seed model provides better fit statistics in these three cases. We list the resulting $\mathrm{p}$-values with test models for the entire sample in Table 4.1 . 

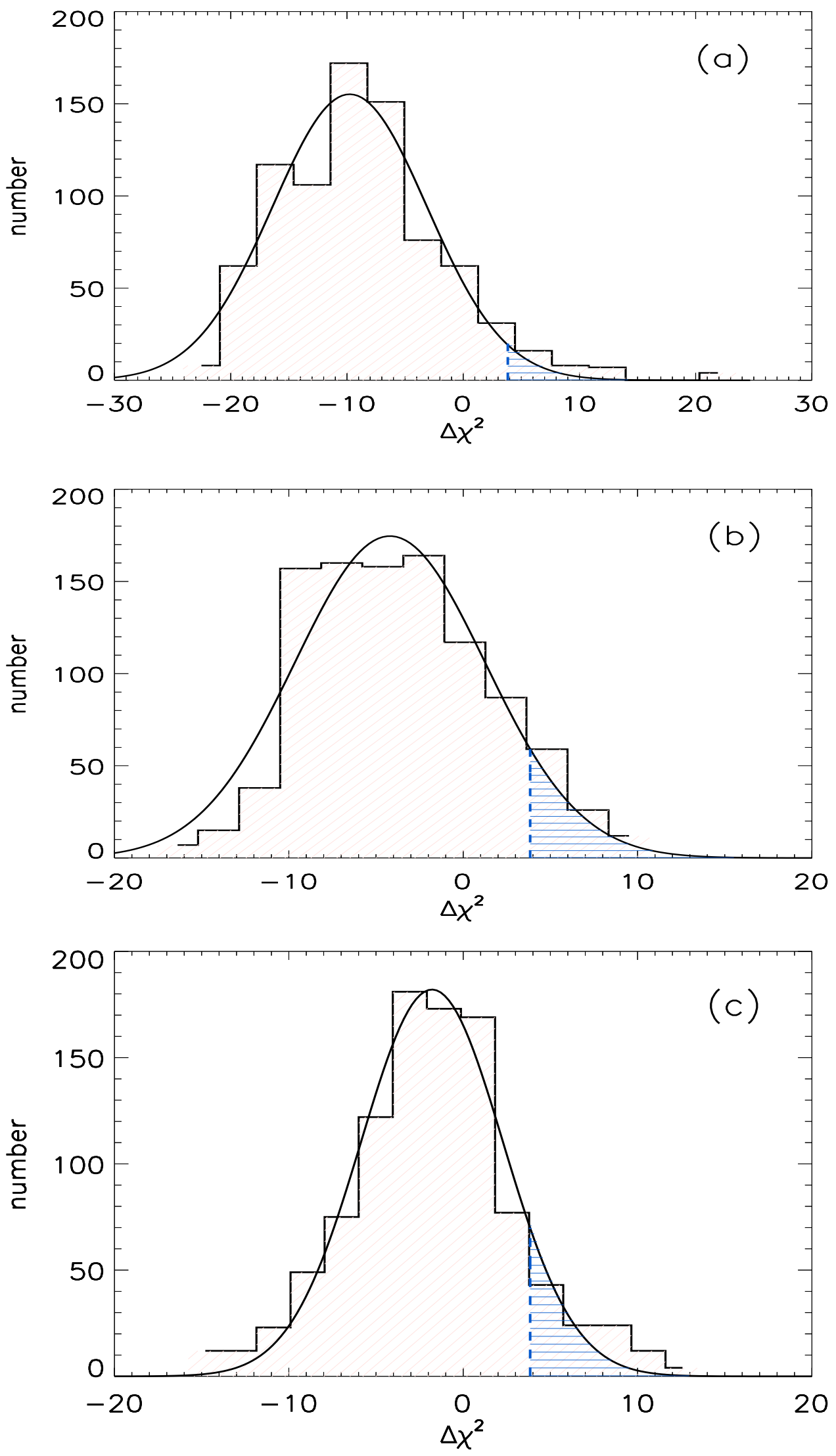

Figure 4.2 Seed Model $\chi^{2}$ - Test Model $\chi^{2}$ distributions for three SGR 1806-20 bursts. Blue shaded regions represent rejection regions of the seed (COMPT) model (where $\Delta \chi^{2}>3.84$ ) (a) $\Delta \chi^{2}=\chi_{\text {COMPT }}^{2}-\chi_{B B+B B}^{2}$ for Burst ID: 328807661. (b) $\Delta \chi^{2}=\chi_{C O M P T}^{2}-\chi_{B B+P O}^{2}$ for Burst ID: 168976265. (c) $\Delta \chi^{2}=\chi_{C O M P T}^{2}-\chi_{L B+L B}^{2}$ for Burst ID: 212194516 . 
Table 4.1. P-values

\begin{tabular}{|c|c|c|c|c|c|c|c|c|c|c|c|}
\hline \multicolumn{3}{|c|}{ SGR J1550-5 } & \multicolumn{3}{|c|}{ SGR $1900+14$} & \multicolumn{3}{|c|}{ SGR 1806-20 } & \multicolumn{3}{|c|}{ SGR 1806-20-continued } \\
\hline $\begin{array}{c}\text { Test } \\
\text { Model }\end{array}$ & $\mathrm{P}$-value & Burst ID & $\begin{array}{c}\text { Test } \\
\text { Model }\end{array}$ & $\mathrm{p}$-value & Burst ID & $\begin{array}{c}\text { Test } \\
\text { Model }\end{array}$ & p-value & Burst ID & $\begin{array}{c}\text { Test } \\
\text { Model }\end{array}$ & p-value & Burst ID \\
\hline $\mathrm{BB}+\mathrm{PO}$ & 0.961 & 475529885 & $\mathrm{BB}+\mathrm{BB}$ & 0.930 & 148213447 & $\mathrm{BB}+\mathrm{BB}$ & 0.961 & 328807661 & $\mathrm{LB}+\mathrm{LB}$ & 0.879 & 330351951 \\
\hline $\mathrm{BB}+\mathrm{PO}$ & 0.984 & 475807758 & $\mathrm{BB}+\mathrm{BB}$ & 0.997 & 236866242 & $\mathrm{BB}+\mathrm{BB}$ & 0.848 & 328974246 & $\mathrm{LB}+\mathrm{LB}$ & 0.910 & 330356347 \\
\hline $\mathrm{LB}+\mathrm{LB}$ & 1.000 & 475537351 & $\mathrm{BB}+\mathrm{BB}$ & 0.921 & 236870495 & $\mathrm{BB}+\mathrm{BB}$ & 0.870 & 329857129 & $\mathrm{LB}+\mathrm{LB}$ & 0.941 & 330357232 \\
\hline $\mathrm{LB}+\mathrm{LB}$ & 1.000 & 476147642 & $\mathrm{BB}+\mathrm{BB}$ & 0.930 & 386236827 & $\mathrm{BB}+\mathrm{BB}$ & 0.996 & 333015699 & $\mathrm{LB}+\mathrm{LB}$ & 1.000 & 330358372 \\
\hline & & & $\mathrm{BB}+\mathrm{PO}$ & 0.895 & 386223878 & $\mathrm{BB}+\mathrm{BB}$ & 0.897 & 333022657 & $\mathrm{LB}+\mathrm{LB}$ & 0.915 & 330364298 \\
\hline & & & $\mathrm{LB}+\mathrm{LB}$ & 0.946 & 146928739 & $\mathrm{BB}+\mathrm{BB}$ & 0.935 & 336043757 & $\mathrm{LB}+\mathrm{LB}$ & 0.926 & 331909545 \\
\hline & & & $\mathrm{LB}+\mathrm{LB}$ & 0.865 & 146929244 & $\mathrm{BB}+\mathrm{BB}$ & 0.887 & 337111577 & $\mathrm{LB}+\mathrm{LB}$ & 0.828 & 331917786 \\
\hline & & & $\mathrm{LB}+\mathrm{LB}$ & 0.939 & 147515660 & $\mathrm{BB}+\mathrm{BB}$ & 0.948 & 349039848 & $\mathrm{LB}+\mathrm{LB}$ & 0.943 & 333015043 \\
\hline & & & $\mathrm{LB}+\mathrm{LB}$ & 0.870 & 147875363 & $\mathrm{BB}+\mathrm{BB}$ & 0.978 & 349063562 & $\mathrm{LB}+\mathrm{LB}$ & 1.000 & 335000025 \\
\hline & & & $\mathrm{LB}+\mathrm{LB}$ & 0.942 & 148387169 & $\mathrm{BB}+\mathrm{BB}$ & 0.881 & 397633576 & $\mathrm{LB}+\mathrm{LB}$ & 0.864 & 335022521 \\
\hline & & & $\mathrm{LB}+\mathrm{LB}$ & 0.952 & 148598029 & $\mathrm{BB}+\mathrm{BB}$ & 0.929 & 403802005 & $\mathrm{LB}+\mathrm{LB}$ & 0.980 & 335364358 \\
\hline & & & $\mathrm{LB}+\mathrm{LB}$ & 0.863 & 230674705 & $\mathrm{BB}+\mathrm{BB}$ & 0.945 & 415917635 & $\mathrm{LB}+\mathrm{LB}$ & 0.928 & 335776010 \\
\hline & & & $\mathrm{LB}+\mathrm{LB}$ & 0.837 & 237656402 & $\mathrm{BB}+\mathrm{BB}$ & 0.985 & 417225091 & $\mathrm{LB}+\mathrm{LB}$ & 0.880 & 335778407 \\
\hline & & & $\mathrm{LB}+\mathrm{LB}$ & 0.919 & 238165998 & $\mathrm{BB}+\mathrm{PO}$ & 0.910 & 168976265 & $\mathrm{LB}+\mathrm{LB}$ & 0.901 & 336985823 \\
\hline & & & $\mathrm{LB}+\mathrm{LB}$ & 0.927 & 257380975 & $\mathrm{BB}+\mathrm{PO}$ & 0.940 & 301268310 & $\mathrm{LB}+\mathrm{LB}$ & 0.909 & 337127242 \\
\hline & & & $\mathrm{LB}+\mathrm{LB}$ & 0.892 & 280822636 & $\mathrm{BB}+\mathrm{PO}$ & 0.919 & 328968241 & $\mathrm{LB}+\mathrm{LB}$ & 0.919 & 337195562 \\
\hline & & & $\mathrm{LB}+\mathrm{LB}$ & 0.938 & 386224496 & $\mathrm{BB}+\mathrm{PO}$ & 0.935 & 329057295 & $\mathrm{LB}+\mathrm{LB}$ & 0.864 & 337887686 \\
\hline & & & $\mathrm{LB}+\mathrm{LB}$ & 0.926 & 386224522 & $\mathrm{BB}+\mathrm{PO}$ & 0.902 & 329059012 & $\mathrm{LB}+\mathrm{LB}$ & 0.873 & 338657332 \\
\hline & & & $\mathrm{LB}+\mathrm{LB}$ & 0.996 & 386224790 & $\mathrm{BB}+\mathrm{PO}$ & 0.893 & 330369519 & $\mathrm{LB}+\mathrm{LB}$ & 0.811 & 339916908 \\
\hline & & & $\mathrm{LB}+\mathrm{LB}$ & 0.955 & 386229747 & $\mathrm{BB}+\mathrm{PO}$ & 0.965 & 330531574 & $\mathrm{LB}+\mathrm{LB}$ & 0.992 & 340351599 \\
\hline & & & $\mathrm{LB}+\mathrm{LB}$ & 0.915 & 386236643 & $\mathrm{BB}+\mathrm{PO}$ & 0.922 & 336044128 & $\mathrm{LB}+\mathrm{LB}$ & 0.860 & 340608599 \\
\hline & & & & & & $\mathrm{BB}+\mathrm{PO}$ & 0.910 & 337103764 & $\mathrm{LB}+\mathrm{LB}$ & 0.934 & 340612616 \\
\hline & & & & & & $\mathrm{BB}+\mathrm{PO}$ & 0.911 & 337194856 & $\mathrm{LB}+\mathrm{LB}$ & 0.944 & 340612697 \\
\hline & & & & & & $\mathrm{BB}+\mathrm{PO}$ & 0.898 & 337200882 & $\mathrm{LB}+\mathrm{LB}$ & 1.000 & 340612733 \\
\hline & & & & & & $\mathrm{BB}+\mathrm{PO}$ & 0.996 & 337201096 & $\mathrm{LB}+\mathrm{LB}$ & 0.988 & 340613037 \\
\hline & & & & & & $\mathrm{BB}+\mathrm{PO}$ & 0.934 & 337511420 & $\mathrm{LB}+\mathrm{LB}$ & 0.926 & 340613750 \\
\hline & & & & & & $\mathrm{BB}+\mathrm{PO}$ & 1.000 & 340612607 & $\mathrm{LB}+\mathrm{LB}$ & 0.969 & 340999953 \\
\hline & & & & & & $\mathrm{BB}+\mathrm{PO}$ & 0.988 & 342756231 & $\mathrm{LB}+\mathrm{LB}$ & 0.875 & 341003003 \\
\hline & & & & & & $\mathrm{BB}+\mathrm{PO}$ & 0.932 & 390182002 & $\mathrm{LB}+\mathrm{LB}$ & 0.983 & 342755100 \\
\hline & & & & & & $\mathrm{LB}+\mathrm{LB}$ & 0.893 & 212194516 & $\mathrm{LB}+\mathrm{LB}$ & 0.916 & 349221450 \\
\hline & & & & & & $\mathrm{LB}+\mathrm{LB}$ & 0.821 & 319514320 & $\mathrm{LB}+\mathrm{LB}$ & 0.866 & 349287908 \\
\hline & & & & & & $\mathrm{LB}+\mathrm{LB}$ & 0.883 & 328027837 & $\mathrm{LB}+\mathrm{LB}$ & 0.942 & 366541462 \\
\hline & & & & & & $\mathrm{LB}+\mathrm{LB}$ & 0.842 & 328815824 & $\mathrm{LB}+\mathrm{LB}$ & 1.000 & 396371066 \\
\hline & & & & & & $\mathrm{LB}+\mathrm{LB}$ & 0.966 & 328894960 & $\mathrm{LB}+\mathrm{LB}$ & 0.891 & 398069005 \\
\hline & & & & & & $\mathrm{LB}+\mathrm{LB}$ & 0.840 & 328898480 & $\mathrm{LB}+\mathrm{LB}$ & 0.978 & 402019525 \\
\hline & & & & & & $\mathrm{LB}+\mathrm{LB}$ & 0.846 & 328973978 & $\mathrm{LB}+\mathrm{LB}$ & 0.875 & 402614229 \\
\hline & & & & & & $\mathrm{LB}+\mathrm{LB}$ & 0.848 & 328984223 & $\mathrm{LB}+\mathrm{LB}$ & 0.921 & 402996582 \\
\hline & & & & & & $\mathrm{LB}+\mathrm{LB}$ & 0.911 & 329064144 & $\mathrm{LB}+\mathrm{LB}$ & 0.867 & 403004861 \\
\hline & & & & & & $\mathrm{LB}+\mathrm{LB}$ & 0.949 & 329330715 & & & \\
\hline
\end{tabular}




\subsubsection{Implications}

Comptonized and thermal emission models are most commonly discussed due to their high fitting power on magnetar bursts (e.g., Lin et al. 2012; van der Horst et al. 2012; Feroci et al. 2004). In the Comptonization viewpoint, the photons emerging from the ignition point are repeatedly upscattered by the $\mathrm{e}^{ \pm}$-pairs present in the corona. The density and optical thickness of the corona and the electron temperature set a spectral turnover point for energy where photons cannot be further heated, realized as the peak energy parameter for the power law shaped Comptonized spectrum. In the context of magnetar bursts, the Comptonized spectrum resembles the models for accretion disks and AGN, but the underlying mechanism differs as a result of the strong magnetic field of magnetars. The corona of hot electrons may emerge in the inner dynamic magnetosphere due to field line twisting as discussed by Thompson et al. 2002b , Thompson \& Beloborodov 2005, Beloborodov \& Thompson 2007 and Nobili et al. 2008. The emergent corona in the magnetosphere could result in a similar Comptonization process and therefore upscatter emergent photons. The anisotropy of the corona sets a different slope for the emission spectrum than discussed above. The emission spectrum however is similar in its exponential tail and peak energy, which is now controlled by the thermal and spatial parameters of the emergent coronas in the magnetosphere. The distinction between persistent and burst emissions are further explained in this model as the bursts may be triggered closer to the surface where the density of $\mathrm{e}^{ \pm}$-pairs is high, and persistent $<10$ $\mathrm{keV}$ signals may originate at higher altitudes with a lower $\mathrm{e}^{ \pm}$density.

\subsection{Relation Between Temperature and Emission Area}

The alternative approach to interpret magnetar burst spectra is the thermal emission due to a shortlasting thermal equilibrium of electron-photon pairs, usually described with the sum of two blackbody functions (see e.g., Feroci et al. 2004; van der Horst et al. 2012). This dual blackbody scheme approximates a continuum temperature gradient due to the total energy dissipation of photons throughout the magnetosphere. The corona is expected to be hotter at low altitudes than the outer layers. Therefore, the coronal structure suggests that the high temperature blackbody component be associated with a smaller volume than the cold component (van der Horst et al. 2012). We report a strong negative correlation between blackbody temperature and emission area, indicating that if a temperature gradient is in fact the underlying emission mechanism, and if the dual blackbody is a well approximation of the continuum gradient as expected, the relationship between temperature and coronal structure is in fact emergent in the spectrum. Modeling the corona causing such temperature gradient is a hard task especially in the hotter zone due to its anisotropic structure, the intense magnetic field, the twisted magnetosphere geometry and polarization-dependence of the 
scattering processes. Temporal and spectral studies on broad energy ranges as a result help get a better view of the underlying structure as well as to distinguish between Comptonized and thermal models.

Although the sum of two blackbody functions is commonly used to describe magnetar burst emission, it was shown that the spectrum of photon flux per unit energy band may be flat at energies lower than the bolometric temperature when the magnetic field is not too high ( $\left.\mathrm{B}<10^{15} \mathrm{G}\right)$. In this model, heretofor referred to as the Lyubarsky (LB) model, the spectrum of photons escaping the bubble formed during the burst are considered. The emergent spectrum was shown by Lyubarsky 2002 to be close to the Blackbody Spectrum although the observed radiation within the bubble comes from photons with different temperatures throughout the bubble. In a hot, optically thick bubble in a strong magnetic field, the photon energy is well below the excitation energy of the first Landau level and Compton scattering dominates. Considering SGR burst bubbles in such a scenario, photons with ordinary orthogonal (O-mode) polarization will go under much more scatterings than the extraordinary linearly polarized (E-mode) photons whose cross-sections are strongly hindered Lyubarsky 2002. Because of this dependence of scatterings on radiation crosssection which in turn depend on frequency in a strong magnetic field, at low energies the burst spectrum alters from the blackbody spectrum and may be observed as flat.

$\mathrm{LB}+\mathrm{LB}$ model emerges as the most frequently chosen test model in our simulations. That is, compared to the other two thermal models with the same degrees of freedom (namely $\mathrm{BB}+\mathrm{PO}$ and $\mathrm{BB}+\mathrm{BB})$, LB model shows the best test statistics for 48 out of 77 bursts with well-constrained parameters for SGR 1806-20, 16 out of 21 for SGR 1900+14,16 and 2 out of 4 for SGR J15505418 (see Figure 4.1). This is suggestive that the modified blackbody scheme with a frequency dependent scattering cross-section modifies the blackbody emission at low energies, and explains burst emission mechanisms better than a thermal emission resulting from short lasting electronpositron pairs through a gradient of temperature.

The overall trend of resulting p-values suggests COMPT as the most plausible model where COMPT provides smaller $\chi^{2}$ values in spectral analysis. Since majority of bursts had better fit statistics with the COMPT model in our spectral analysis, our results indicate a non-thermal Comptonized character of emission spectrum more favorable than thermal emission. These results differ significantly from the results of Lin et al. 2012 where a thermal character was suggested to better describe SGR J1550-5418 bursts. However, BB+BB was not selected as the test model in any of our simulations for SGR J1550-5418. It remains possible that if a different test model was selected with a different method, or if new bursts data provides with well-constrained parameters with lower $\chi^{2}$ values resulting in different choices of test models, it may provided significantly better fits than COMPT for at least some events. 


\subsubsection{Correlations}

In the thermal emission viewpoint, a temperature gradient throughout the magnetar surface is assumed. This gradient from the burst ignition point has been shown in previous studies to be well described by a hot and cool blackbody components. Using the blackbody model, we have found a significant anti-correlation between emission areas and temperatures of hot and cold blackbody components. We only considered unsaturated bursts with well-constrained parameters to calculate emission areas as following:

$$
R^{2}=F D^{2} / \sigma T^{4}
$$

where $\mathrm{R}$ is the emission radius, $\mathrm{D}$ is the distance to source and $\mathrm{F}$ is the average total flux per event, (fluence multiplied by exposure time summed for PCA and HXT divided by total exposure time).

In the upper left panel of Figure 4.3 we plot $R^{2}\left(\mathrm{~km}^{2}\right)$ vs. $\mathrm{kT}(\mathrm{keV})$ for all three sources with unsaturated bursts with well-constrained parameters. The dotted lines represent $R^{2} \propto T^{-3}$ and $R^{2} \propto T^{-4}$ values scaled here for visual comparison only. The cool blackbody components (kT1, shown in black) are seperated from the hot blackbody (kT2, shown in red) components by higher emission area for SGR 1806-20, SGR 1900+14 and SGR J1550-5418 collectively, similar to those reported by Van der Horst et al. (2021) and Lin et al. (2011) for SGR J1550-5418. This supports the thermal emission model with temperature gradient starting from the hot and narrower ignition point to a wider area where it is cooled down. We employed a Spearman Rank Correlation test on emission area vs. blackbody temperature and found a correlation coefficient of -0.933 with a chance of probability less than $10^{-37}$ using all three sources. The correlation coefficients are $-0.953,-0.962,-0.942$ when the Spearman Correlation test is employed individually for SGR J1550-5418, SGR 1900+14 and SGR 1806-20 respectively. The tests showed a chance of probability of $8.45 \times 10^{-31}$ for SGR J1550-5418 and less than $10^{-37}$ for the other two sources. These results imply a strongly negative correlation between emission area and temperature for all sources investigated, as the $\mathrm{BB}+\mathrm{BB}$ model for a temperature gradient for thermal emission suggests.

\subsubsection{Power Indexes and Relationship with Flux}

In order to check whether the given correlation holds the same properties for different flux levels, we grouped the data into 3 flux intervals and employed a broken power law fit for each group in the same flux interval individually. We report the color-coded scatterplot of different flux values for SGR 1806-20 in the upper right panel of Figure 4.3. The dotted lines represent the broken power law fits and the data points on kT axis represent the break index values colored according to each flux group. The fit result show an increasing trend of power index and $k T_{\text {break }}$ with respect to flux. 
Table 4.2. Broken Power Law Fit Results with Corresponding Flux Intervals

\begin{tabular}{cccccc}
\hline \hline Source & $\begin{array}{c}\log _{10} \text { Flux } \\
\text { Interval }\left(\text { ergs } / \mathrm{s} / \mathrm{cm}^{2}\right)\end{array}$ & $\begin{array}{c}\text { Power Index } \\
\text { Below } k T_{\text {break }}\end{array}$ & $\begin{array}{c}\text { Power Index } \\
\text { Above } k T_{\text {break }}\end{array}$ & $\begin{array}{c}k T_{\text {break }} * \\
(\mathrm{keV})\end{array}$ & $\chi^{2} / D O F$ \\
\hline SGR 1806-20 & $-8.31,-7.91$ & $-3.79 \pm 0.03$ & $-3.78 \pm 0.03$ & 5.26 & 0.93 \\
SGR 1806-20 & $-7.91,-7.77$ & $-4.04 \pm 0.03$ & $-3.91 \pm 0.04$ & 7.54 & 0.4 \\
SGR 1806-20 & $-7.77,-7.26$ & $-7.59 \pm 0.02$ & $-5.57 \pm 0.04$ & 8.08 & 0.87 \\
SGR 1900+14 & $-8.4,-7.16$ & $-5.44 \pm 0.01$ & $-4.00 \pm 0.04$ & 6.01 & 2.12 \\
SGR J1550-25418 & $-8.43,-7.58$ & $-3.55 \pm 0.03$ & $-3.43 \pm 0.03$ & 7.58 & 0.92 \\
\hline
\end{tabular}

We note that the relation between $\mathrm{kT}$ and area is similar at flux values below $10^{-7.9} \mathrm{erg} \mathrm{cm}^{-2} \mathrm{~s}^{-1}$ for cool and hot blackbody components. With increasing flux, the relation between area and kT varies between the blackbody components, area decreasing more steeply for the cooler blackbody.

Since SGR J1550-5418 and SGR 1900+14 has fewer unsaturated bursts with well-constrained parameters, it is hard to distinguish increasing flux with a color-coded plot. Therefore, we have employed a broken-power law fit on SGR J1550-5418 (Figure 4.3, lower left panel) and SGR 1900+14 (Figure 4.3, lower right panel) without flux grouping. The broken power law fits (shown in red) for SGR 1900+14 show a significant change in steepness between two blackbody components. Similar to the results of SGR1806-20, SGR 1900+14 bursts show a steeper area decrease for the cooler blackbody component. For SGR J1550-5418, the power indices agree with each other within errors, suggesting a simple power law model (shown in black dashed line) may well describe the relation between area and kT with a power index of -3.29 and a reduced $\chi^{2}$ of 0.88 . We report the resulting broken power law indices with level of significance information ( $\left.\chi^{2} / D O F\right)$ on Table 4.2 for all three sources.

These relations between emission area and temperature significantly differs from the relation discussed in Younes et al. 2014 where the area decreases more steeply for the second blackbody component with increasing temperature. However, we note that the flux range analyzed by Younes et. al. for SGR J1550-5418 (above $10^{-6.5} \mathrm{erg} \mathrm{cm}^{-2} \mathrm{~s}^{-1}$ ) is much higher from the flux range covered in this analysis for SGR 1806-20 (below $1.03 \times 10^{-8} \mathrm{erg} \mathrm{cm}^{-2} \mathrm{~s}^{-1}$ ), SGR 1900+14 (below $6.93 \times$ $10^{-8} \mathrm{erg} \mathrm{cm}^{-2} \mathrm{~s}^{-1}$ ) and SGR J1550-5418 (below $2.62 \times 10^{-8} \mathrm{ergs} / \mathrm{cm}^{2} / \mathrm{sec}$ ). This suggests the steepness of area vs. kT behaviours of the two blackbody components may differ more significantly with increasing flux than previously discussed. It is also possible that the relation is variant between different sources, given the difference between power law indexes as well as the relation between power law indexes of blackbody components between the three sources discussed. However, it is important to note that SGR J1550-5418 and the first group (with smallest flux) of SGR 1806-20 contain similar flux regimes and show a similar relationship between the two power law indexes (power law indexes agree with each other within errors for the two blackbody components). 
The overall trend of power indexes suggests the steepness of area vs. kT behaviours of the two blackbody components differ more significantly with increasing flux than previously discussed. It is also possible that the trend between lower and upper power law indices revert with burst intensity. We suggest that the power law trends of the cooler and hot components are the same in the flux regime $\sim 10^{-8} \mathrm{erg} \mathrm{cm}^{-2} \mathrm{~s}^{-1}$. Above this flux level, the cooler component shows a steeper trend than that of the hot component. In the much higher flux regime (above $\sim 10^{-7} \mathrm{erg} \mathrm{cm}^{-2} \mathrm{~s}^{-1}$ ), an opposite behaviour takes place, as presented by Younes et al. (2014); namely, the emission area of the hot component drops steeper than that of the cooler component. 

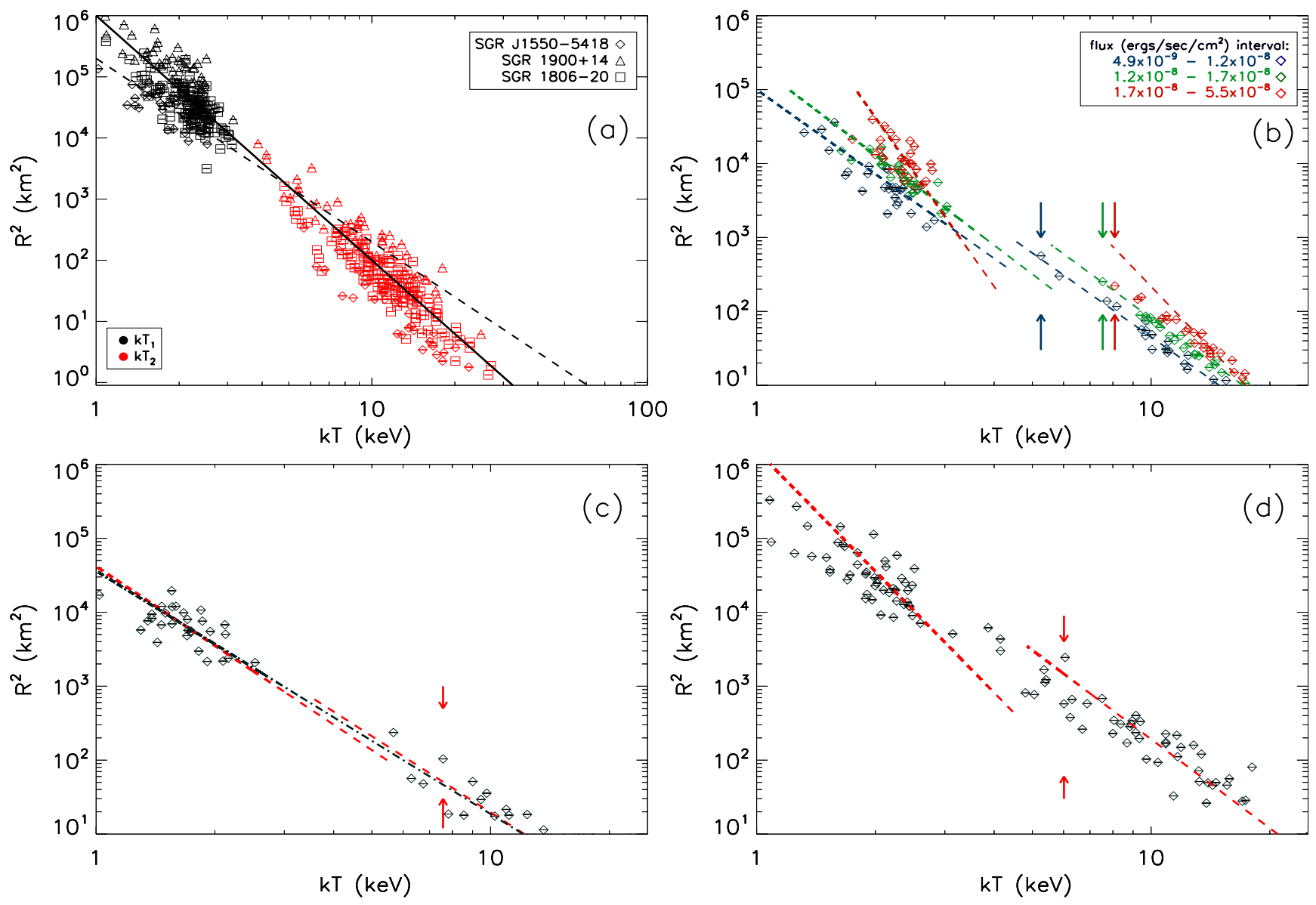

Figure 4.3 Upper left panel (a) SGR J1550-5418, SGR 1900+14, SGR 1806-20 emission area vs. hot and cold blackbody temperatures. $R^{2} \propto T^{-3}$ and $R^{2} \propto T^{-4}$ are drawn with dashed and solid lines respectively for comparison only. Upper right panel (b) SGR 1806-20 emission area vs. hot and cold blackbody temperatures grouped by total flux values with corresponding broken power law fits. Break index in $\mathrm{kT}$ space $(\mathrm{keV})$ are shown with color-coded arrows. Lower left panel (c) SGR J1550-5814 emission area vs. hot and cold blackbody temperatures with broken power law (red dashed line) and linear model (black dashed line) fits. Arrows represent break index in kT space (keV). Lower right panel (d) SGR 1900+14 emission area vs. hot and cold blackbody temperatures with broken power law fit shown in dashed line. Break index in $\mathrm{kT}$ space $(\mathrm{keV})$ is shown with the arrows. 


\section{Chapter 5}

\section{Summary and Conclusions}

In this thesis, we performed broadband $(2-250 \mathrm{keV})$ spectral analysis of the bursts of three magnetars: SGR J1550-5418, SGR 1900+14 and SGR 1806-20 observed with the RXTE (Rossi X-ray Timing Explorer) mission. We used joint spectra of PCA and HEXTE instrument of RXTE, resulting in an energy range coverage of 2-250 keV, which is the broadest coverage on SGR 1806-20 to date. We took sum of two blackbody functions $(B B+B B)$, sum of blackbody and power law functions (BB+PO), Comptonizated (COMPT) and sum of two modified blackbody functions (LB+LB), which is employed in spectral analysis for the first time in this study, as our models. We also calculated unabsorbed fluxes for these sources based on each model, and presented detailed statistical results for all sources and parameter, flux and energy distributions where the burst sample size was large enough (i.e. for SGR 1900+14 and SGR 1806-20). Our spectral analysis yielded that the majority of bursts have lower reduced $\chi^{2}$ values when fit by the COMPT model.

The three thermal models $(\mathrm{BB}+\mathrm{BB}, \mathrm{BB}+\mathrm{PO}$ and $\mathrm{LB}+\mathrm{LB})$ used in our analysis have equal number of parameters, resulting in equal degrees of freedom for fit purposes. Therefore, it is possible to employ a simple $\Delta \chi^{2}$ test to compare these models. A simple $\Delta \chi^{2}$ test with enforced parameter error restrictions (i.e. requiring error $<50 \%$ of the parameter) yields that $\mathrm{LB}+\mathrm{LB}$ explains burst spectra best within thermal models for 66 out of 102 bursts with well-restricted parameters. However, COMPT model involves one less free parameter than the thermal models and therefore a simple $\Delta \chi^{2}$ test was not sufficient to compare COMPT with the remaining thermal models. We therefore performed extensive simulations on each burst with well-restricted $(<50 \%$ of the parameter) errors. For each burst, we simulated 1000 burst spectra based on the COMPT fits and fit the spectra with COMPT and the test model. We chose the test model to be the model that yields the lowest reduced $\chi^{2}$ value and also has well-restricted parameters. If more than $90 \%$ of simulated burst spectra were better fit with COMPT within the significance level 0.05 , we concluded that COMPT provides better fits for the burst. Overall, COMPT was preferred for in $67.6 \%$ of bursts.

Based on the simulation results, we suggest COMPT provides significantly better fits when it 
is the inherent dominant emission mechanism. Since the majority bursts in our spectral analysis were better fit with the COMPT model, we suggest the inherent emission mechanism is likely nonthermal for majority of bursts included in our study within $2-250 \mathrm{keV}$ range. For the remaining bursts that are better fit with thermal models, we suggest LB+LB model emerges as the best fitting thermal model overall.

We then searched for correlations between fitted parameters. We found a significant anticorrelation between blackbody temperatures (hot and cold) and emission areas. Overall, emission area decreases with increasing blackbody temperature in all cases, supporting the thermal emission model from a hot bubble where emission radiates from a hot ignition point to a colder and wider emission area gradually. We then grouped our burst sample into three flux intervals and checked if the relation between emission area and temperature differs for different flux intervals and between the hot and cool blackbody components. We fit a broken power law model on each flux group for all three sources. The overall trend of power indexes of cool and hot blackbody components suggests a similar blackbody temperature vs. emission area relationship for the cool and hot blackbodies in the flux regime $\sim 10^{-8} \mathrm{erg} \mathrm{cm}^{-2} \mathrm{~s}^{-1}$. Above this flux regime, the steepness for the cool blackbody component increases much faster (i.e. emission area decreases more rapidly with respect to temperature for the cooler blackbody). Our results significantly differ from Younes et al. 2014, whose results suggest the hotter blackbody component shows a steeper increase in much higher flux regimes (above $\sim 10^{-7} \mathrm{erg} \mathrm{cm}^{-2} \mathrm{~s}^{-1}$ ). We suggest the steepness of area vs. kT behaviours of the two blackbody components may differ more significantly with increasing flux than previously discussed. It is also possible that the trend between lower and upper power law indices revert with burst intensity. 


\section{Bibliography}

Alpar, M. A. 1999, ArXiv Astrophysics e-prints

-. 2001, ApJ, 554, 1245

Alpar, M. A., Cheng, A. F., Ruderman, M. A., \& Shaham, J. 1982, Nature, 300, 728

Beloborodov, A. M. \& Thompson, C. 2007, ApSS, 308, 631

Şaşmaz Muş, S., Aydın, B., \& Göğüş, E. 2014, MNRAS, 440, 2916

Chadwick, J. 1932, Proceedings of the Royal Society of London Series A, 136, 692

Chatterjee, P. \& Hernquist, L. 2000, ApJ, 543, 368

Chatterjee, P., Hernquist, L., \& Narayan, R. 2000, ApJ, 534, 373

Ertan, Ü. \& Alpar, M. A. 2003, ApJL, 593, L93

Esposito, P., Mereghetti, S., Tiengo, A., Zane, S., Turolla, R., Götz, D., Rea, N., Kawai, N., Ueno, M., Israel, G. L., Stella, L., \& Feroci, M. 2007, AAP, 476, 321

Fenimore, E. E., Laros, J. G., \& Ulmer, A. 1994, ApJ, 432, 742

Feroci, M., Caliandro, G. A., Massaro, E., Mereghetti, S., \& Woods, P. M. 2004, ApJ, 612, 408

Hartman, J. M., Patruno, A., Chakrabarty, D., Markwardt, C. B., Morgan, E. H., van der Klis, M., \& Wijnands, R. 2009, ApJ, 702, 1673

Hewish, A., Bell, S. J., Pilkington, J. D. H., Scott, P. F., \& Collins, R. A. 1968, Nature, 217, 709

Hewish, A. \& Okoye, S. E. 1965, Nature, 207, 59

Ikhsanov, N. R. 2007, MNRAS, 375, 698

Illarionov, A. F. \& Kompaneets, D. A. 1990, MNRAS, 247, 219

Israel, G. L., Romano, P., Mangano, V., Dall'Osso, S., Chincarini, G., Stella, L., Campana, S., Belloni, T., Tagliaferri, G., Blustin, A. J., Sakamoto, T., Hurley, K., Zane, S., Moretti, A., Palmer, D., Guidorzi, C., Burrows, D. N., Gehrels, N., \& Krimm, H. A. 2008, ApJ, 685, 1114

Jahoda, K., Markwardt, C. B., Radeva, Y., Rots, A. H., Stark, M. J., Swank, J. H., Strohmayer, T. E., \& Zhang, W. 2006, ApJS, 163, 401 
Kaspi, V. M. \& Beloborodov, A. 2017, ArXiv e-prints

Landau, L. D. 1932, Phys. Z, 285, 1

Lattimer, J. M. 2012, Annual Review of Nuclear and Particle Science, 62, 485

Lin, L., Göğüş, E., Baring, M. G., Granot, J., Kouveliotou, C., Kaneko, Y., van der Horst, A., Gruber, D., von Kienlin, A., Younes, G., Watts, A. L., \& Gehrels, N. 2012, ApJ, 756, 54

Lin, L., Göğüş, E., Kaneko, Y., \& Kouveliotou, C. 2013, ApJ, 778, 105

Lyubarsky, Y. E. 2002, MNRAS, 332, 199

Lyutikov, M. 2003, MNRAS, 346, 540

Manchester, R. N., Hobbs, G. B., Teoh, A., \& Hobbs, M. 2005, AJ, 129, 1993

Mazets, E. P., Golenetskij, S. V., \& Guryan, Y. A. 1979, Soviet Astronomy Letters, 5, 641

Mereghetti, S., Pons, J. A., \& Melatos, A. 2015, SSR, 191, 315

Motch, C., Zavlin, V. E., \& Haberl, F. 2003, AAP, 408, 323

Nakagawa, Y. E., Yoshida, A., Hurley, K., Atteia, J.-L., Maetou, M., Tamagawa, T., Suzuki, M., Yamazaki, T., Tanaka, K., Kawai, N., Shirasaki, Y., Pelangeon, A., Matsuoka, M., Vanderspek, R., Crew, G. B., Villasenor, J. S., Sato, R., Sugita, S., Kotoku, J., Arimoto, M., Pizzichini, G., Doty, J. P., \& Ricker, G. R. 2007, PASJ, 59, 653

Nobili, L., Turolla, R., \& Zane, S. 2008, MNRAS, 389, 989

Olive, J.-F., Hurley, K., Dezalay, J.-P., Atteia, J.-L., Barraud, C., Butler, N., Crew, G. B., Doty, J., Ricker, G., Vanderspek, R., Lamb, D. Q., Kawai, N., Yoshida, A., Shirasaki, Y., Sakamoto, T., Tamagawa, T., Torii, K., Matsuoka, M., Fenimore, E. E., Galassi, M., Tavenner, T., Donaghy, T. Q., \& Graziani, C. in , American Institute of Physics Conference Series, Vol. 662, GammaRay Burst and Afterglow Astronomy 2001: A Workshop Celebrating the First Year of the HETE Mission, ed. G. R. RickerR. K. Vanderspek, 82-87

Oppenheimer, J. R. \& Volkoff, G. M. 1939, Physical Review, 55, 374

Özel, F. \& Freire, P. 2016, ARAA, 54, 401

Paczynski, B. 1990, ApJL, 365, L9

Rhoades, C. E. \& Ruffini, R. 1974, Physical Review Letters, 32, 324

Sasmaz Mus, S., Kaneko, Y., Kirmizibayrak, D., \& Gogus, E. in preparation

Scargle, J. D., Norris, J. P., Jackson, B., \& Chiang, J. 2013, ApJ, 764, 167

Slane, P. O., Helfand, D. J., \& Murray, S. S. 2002, ApJL, 571, L45

Thompson, C. \& Beloborodov, A. M. 2005, ApJ, 634, 565 
Thompson, C. \& Duncan, R. C. 1995, MNRAS, 275, 255

-. 2001, ApJ, 561, 980

Thompson, C., Duncan, R. C., Woods, P. M., Kouveliotou, C., Finger, M. H., \& van Paradijs, J. 2000, ApJ, 543, 340

Thompson, C., Lyutikov, M., \& Kulkarni, S. R. 2002a, ApJ, 574, 332

-. 2002b, ApJ, 574, 332

Tolman, R. C. 1939, Physical Review, 55, 364

Turolla, R., Zane, S., \& Watts, A. L. 2015, Reports on Progress in Physics, 78, 116901

van der Horst, A. J., Kouveliotou, C., Gorgone, N. M., Kaneko, Y., Baring, M. G., Guiriec, S., Göğüss, E., Granot, J., Watts, A. L., Lin, L., Bhat, P. N., Bissaldi, E., Chaplin, V. L., Finger, M. H., Gehrels, N., Gibby, M. H., Giles, M. M., Goldstein, A., Gruber, D., Harding, A. K., Kaper, L., von Kienlin, A., van der Klis, M., McBreen, S., Mcenery, J., Meegan, C. A., Paciesas, W. S., Pe'er, A., Preece, R. D., Ramirez-Ruiz, E., Rau, A., Wachter, S., Wilson-Hodge, C., Woods, P. M., \& Wijers, R. A. M. J. 2012, ApJ, 749, 122

van Kerkwijk, M. H., Breton, R. P., \& Kulkarni, S. R. 2011, ApJ, 728, 95

Webb, N. A. \& Barret, D. 2007, ApJ, 671, 727

Weisskopf, M. C., O’Dell, S. L., Paerels, F., Elsner, R. F., Becker, W., Tennant, A. F., \& Swartz, D. A. 2004, ApJ, 601, 1050

Xu, R. 2007, Advances in Space Research, 40, 1453

Yakovlev, D. G. \& Pethick, C. J. 2004, ARAA, 42, 169

Younes, G., Kouveliotou, C., van der Horst, A. J., Baring, M. G., Granot, J., Watts, A. L., Bhat, P. N., Collazzi, A., Gehrels, N., Gorgone, N., Göğüsş, E., Gruber, D., Grunblatt, S., Huppenkothen, D., Kaneko, Y., von Kienlin, A., van der Klis, M., Lin, L., Mcenery, J., van Putten, T., \& Wijers, R. A. M. J. 2014, ApJ, 785, 52 